\title{
Aspects of Nonlinear Dielectric Spectroscopy in the Noninvasive Determination of Glucose
}

by

\author{
Christopher Jarl McLellan
}

A thesis submitted to the Faculty of Graduate Studies and Research

in partial fulfillment of the requirements for the degree of

\author{
Master of Applied Science \\ in
}

\section{Electrical Engineering}
Ottawa-Carleton Institute for Electrical and Computer Engineering Department of Systems and Computer Engineering
Carleton University
Ottawa, Ontario
Canada
January, 2007

(c) Christopher Jarl McLellan, 2007 


$\begin{array}{ll}\begin{array}{l}\text { Library and } \\ \text { Archives Canada }\end{array} & \begin{array}{l}\text { Bibliothèque et } \\ \text { Archives Canada }\end{array} \\ \begin{array}{l}\text { Published Heritage } \\ \text { Branch }\end{array} & \begin{array}{l}\text { Direction du } \\ \text { Patrimoine de l'édition }\end{array} \\ \begin{array}{l}\text { 395 Wellington Street } \\ \text { Ottawa ON K1A ON4 }\end{array} & \begin{array}{l}\text { 395, rue Wellington } \\ \text { Ottawa ON K1A ON4 } \\ \text { Canada }\end{array}\end{array}$

Your file Votre référence ISBN: 978-0-494-23341-2 Our file Notre référence ISBN: 978-0-494-23341-2

NOTICE:

The author has granted a nonexclusive license allowing Library and Archives Canada to reproduce, publish, archive, preserve, conserve, communicate to the public by telecommunication or on the Internet, loan, distribute and sell theses worldwide, for commercial or noncommercial purposes, in microform, paper, electronic and/or any other formats.

The author retains copyright ownership and moral rights in this thesis. Neither the thesis nor substantial extracts from it may be printed or otherwise reproduced without the author's permission.
AVIS:

L'auteur a accordé une licence non exclusive permettant à la Bibliothèque et Archives Canada de reproduire, publier, archiver, sauvegarder, conserver, transmettre au public par télécommunication ou par l'Internet, prêter, distribuer et vendre des thèses partout dans le monde, à des fins commerciales ou autres, sur support microforme, papier, électronique et/ou autres formats.

L'auteur conserve la propriété du droit d'auteur et des droits moraux qui protège cette thèse. $\mathrm{Ni}$ la thèse ni des extraits substantiels de celle-ci ne doivent être imprimés ou autrement reproduits sans son autorisation.
In compliance with the Canadian

Privacy Act some supporting forms may have been removed from this thesis.

While these forms may be included in the document page count, their removal does not represent any loss of content from the thesis.
Conformément à la loi canadienne sur la protection de la vie privée, quelques formulaires secondaires ont été enlevés de cette thèse.

Bien que ces formulaires aient inclus dans la pagination, il n'y aura aucun contenu manquant.

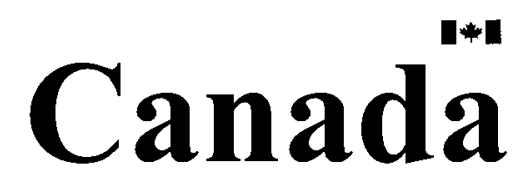




\section{Abstract}

Diabetes is a chronic disease of the metabolic system with serious health and social implications. Research in noninvasive methods to measure blood glucose levels is ongoing as a means to improve the monitoring techniques necessary for the management of this disease.

In this dissertation the technique of nonlinear dielectric spectroscopy (NLDS) is evaluated as a noninvasive method in measuring glucose levels. NLDS injects a low frequency probing current into a sample, and obtains meaningful information through the analysis of system nonlinearity. A suspension of the yeast $S$. cerevisiae is used in the experimental NLDS work, and metabolically active and quiescent yeast states are considered, as well as the electrolyte medium. Experimental time-course spectral data are presented. Electrode polarization artifacts are examined as masking the biological signal of interest. An empirical model is fitted to the experimental data at discrete points over time as a method to compensate for artifacts. 


\section{Acknowledgements}

First and foremost I would like to thank my supervisors, Adrian Chan and Rafik Goubran for their guidance, support, and encouragement.

This work was financially supported by Ontario Centres of Excellence - Communications and Information Technology Ontario division, and the Department of Systems and Computer Engineering, Carleton University. Biopeak Corp. of Ottawa, Canada supported this work with expertise and laboratory facilities.

I would like to thank Andria Dawson and Craig Millen for their much appreciated help and wisdom, and for the many discussions regarding this work.

Finally, I would like to thank my family, especially my parents John and Judy McLellan, for their patience and support. 


\section{Contents}

Abstract

Acknowledgements

iv

List of Tables

List of Figures $\quad$ xii

List of Symbols $\quad$ xv

1 Introduction 1

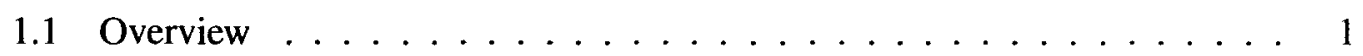

1.2 Problem of Interest ........................ 2

1.3 Objectives and Goals $\ldots \ldots \ldots \ldots \ldots$

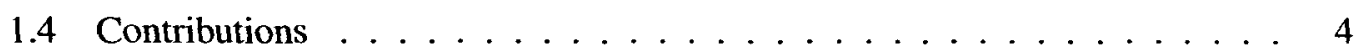

1.5 Outline of the Dissertation $\ldots \ldots \ldots \ldots$

2 Background $\quad 8$

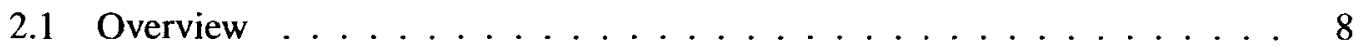

$$
\text { v }
$$




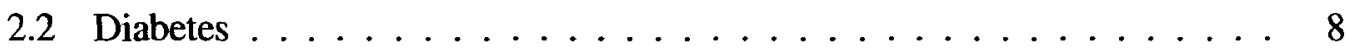

2.3 Current Research in Noninvasive Methods . . . . . . . . . . . . . . . 11

2.3.1 Near-Infrared Spectroscopy . . . . . . . . . . . . . . . . . 11

2.3.2 Raman Spectroscopy . . . . . . . . . . . . . . . 12

2.3 .3 Polarimetry .................... 13

2.3 .4 Photacoustics . . . . . . . . . . . . . . 14

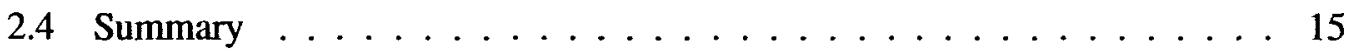

3 Nonlinear Dielectric Spectroscopy 17

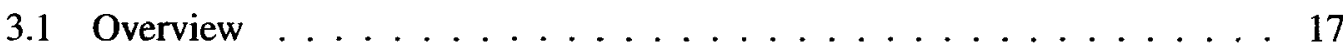

3.2 Dielectric Theory Applicable to Biological

Cells . . . . . . . . . . . . . . . . . . . 18

3.3 Biological Response . . . . . . . . . . . . . . . . . . . 21

3.3.1 Transport Enzymes . . . . . . . . . . . . . . . 21

3.4 Fundamental Work in NLDS . . . . . . . . . . . . . . . . . 24

3.4 .1 Voltage Clamp Work $\ldots \ldots \ldots \ldots . \ldots . \ldots 25$

3.4 .2 Current Clamp Work . . . . . . . . . . . . . . . 27

3.5 Electrode-Electrolyte System . . . . . . . . . . . . . 28

3.5.1 Electrode Polarization . . . . . . . . . . . . . . . . . . . 29

vi 
3.5.2 Polarizable and Nonpolarizable Electrodes . . . . . . . . . . . 30

3.5.3 Electrode Types in NLDS . . . . . . . . . . . . . . 30

3.5.4 Electrode Nonlinear Artifact Minimization . . . . . . . . . . . . . 31

3.6 Signal Analysis and Processing . . . . . . . . . . . . . . 32

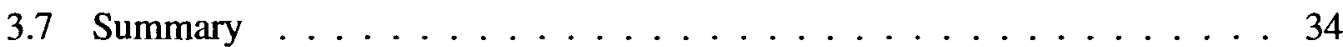

4 NLDS Experimental Work $\quad 35$

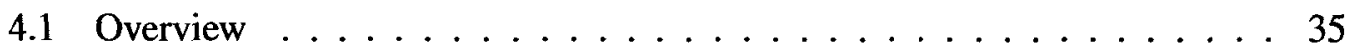

4.2 Materials and Methods $\ldots \ldots \ldots \ldots$. . . . . . . . . . . . . .

$4.2 .1 \quad$ Apparatus ........................ 36

4.2.2 Medium and Cell Suspension . . . . . . . . . . . . . . 38

4.2 .3 Signal Processing . . . . . . . . . . . . . . 38

4.3 Experimental Test Cases $\ldots \ldots \ldots \ldots . \ldots \ldots$

4.3.1 Electrolyte Medium . . . . . . . . . . . . . . . . . . . 39

4.3.2 Quiescent Yeast Suspension . . . . . . . . . . . . . . 39

4.3 .3 Active Yeast Suspension . . . . . . . . . . . . . . . 40

4.3.4 Boiled Active Yeast Suspension _ . . . . . . . . . . . . 40

4.3.5 Electrolyte Medium and Glucose . . . . . . . . . . . . . 40

4.4 Sequential Experimental Work . . . . . . . . . . . . . . . . . . . 41

vii 
$4.4 .1 \quad$ Experimental Protocol $\ldots \ldots \ldots \ldots . \ldots . \ldots 41$

4.4 .2 Sequential Experimental Results . . . . . . . . . . . . . 42

4.4 .3 Discussion of Results . . . . . . . . . . . . . . . . 49

4.5 Parallel Experimental Work . . . . . . . . . . . . . . . 50

4.5 .1 Improvements . . . . . . . . . . . . . 50

4.5.2 Parallel Experimental Results . . . . . . . . . . . . 52

4.5.3 Discussion of Results . . . . . . . . . . . . . 66

$4.6 \mathrm{Ag}-\mathrm{AgCl}$ Electrode Experimental Work $\ldots \ldots \ldots 70$

4.6.1 Improvements . . . . . . . . . . . . 70

4.6.2 Ag-AgCl Electrode Experimental Results . . . . . . . . . . 71

4.6 .3 Discussion of Results . . . . . . . . . . . . . . . 86

4.7 Chapter Summary . . . . . . . . . . . . . . . . . . 87

5 Analysis of Experimental Results

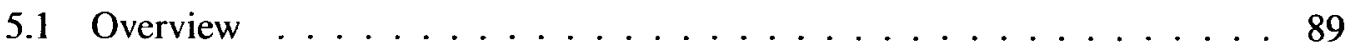

5.2 Circuit Model . . . . . . . . . . . . . . . . . 90

$5.2 .1 \quad$ Electrode Model $\ldots \ldots \ldots . \ldots . \ldots 90$

5.2 .2 Drive Circuit Model . . . . . . . . . . . . . . . . . 93

5.3 Electrode Geometry Work . . . . . . . . . . . . . . 97

viii 
5.3.1 Evaluation of Ohmic Impedance . . . . . . . . . . . 97

5.3.2 Coupling of Interfacial and Ohmic Impedance . . . . . . . . . 100

5.3.3 Effect of Cellular Bulk . . . . . . . . . . . . . 101

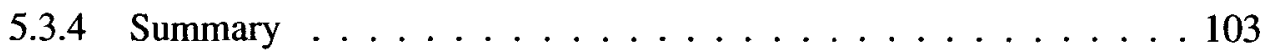

5.4 Current Variability . . . . . . . . . . . . . . . . . . 103

5.4 .1 Average Power Analysis . . . . . . . . . . . . . . . 104

5.5 Metabolic Byproducts . . . . . . . . . . . . . . 106

5.5.1 Conductivity and $\mathrm{CO}_{2} \ldots \ldots \ldots \ldots \ldots$

5.5.2 Bubble Production and $\mathrm{CO}_{2} \ldots \ldots \ldots \ldots \ldots$

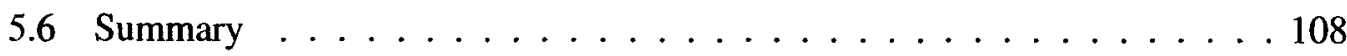

6 Artifact Compensation Methods 110

6.1 Overview ............................. 110

6.2 System Model . . . . . . . . . . . . . . . . . . . . . . 111

6.3 A Compensation Method for Variation in

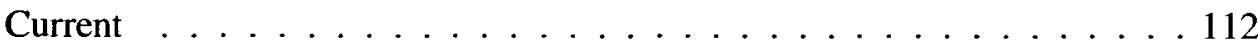

6.3.1 Current Variation Compensation Results . . . . . . . . . . . . 115

6.4 A Method to Compensate for Electrode

Polarization . . . . . . . . . . . . . . . 118

6.4.1 Polarization Compensation Results . . . . . . . . . . . . . 122

ix 


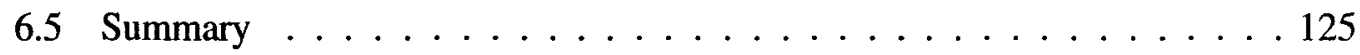

$\begin{array}{llr}7 & \text { Discussion } & 127\end{array}$

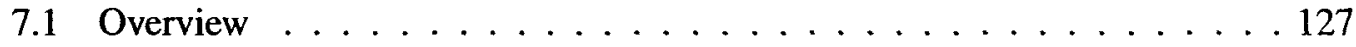

7.2 Thesis Work . . . . . . . . . . . . . . . . . . 127

7.3 Effect of Cellular Bulk _. . . . . . . . . . . . . . . . . . . 129

7.4 Compensation Methods . . . . . . . . . . . . . . 130

7.5 NLDS As a Noninvasive Method . . . . . . . . . . . . . . 131

7.6 Future Work . . . . . . . . . . . . . . . . . . 132

7.7 Summary . . . . . . . . . . . . . . . . . . . 134

$\begin{array}{ll}\text { References } & 136\end{array}$ 


\section{List of Tables}

4.1 Response harmonics means and standard deviations . . . . . . . . . 80

4.2 Drive harmonics means and standard deviations $\ldots \ldots \ldots$. . . . . 81

4.3 Correlation coefficients $\ldots \ldots \ldots \ldots \ldots$ 


\section{List of Figures}

3.1 Four electrode NLDS apparatus . . . . . . . . . . . . . . . . . . 24

4.1 NLDS experimental apparatus . . . . . . . . . . . . . 37

4.2 PSD of electrolyte response $\ldots \ldots \ldots \ldots \ldots$

4.3 PSD of electrolyte drive $\ldots \ldots \ldots \ldots \ldots \ldots$

4.4 PSD of quiescent yeast response $\ldots \ldots \ldots \ldots \ldots$

4.5 PSD of quiescent yeast drive $\ldots \ldots \ldots \ldots \ldots \ldots$

4.6 PSD of active yeast response $\ldots \ldots \ldots \ldots \ldots \ldots$

4.7 PSD of active yeast drive $\ldots \ldots \ldots \ldots \ldots \ldots$

4.8 Quiescent yeast reference spectrum results . . . . . . . . . . . . 48

4.9 Active yeast reference spectrum results . . . . . . . . . . . . . 48

4.10 Three dimensional time-frequency representation $\ldots \ldots \ldots 53$

4.11 Electrolyte response time-course spectral results . . . . . . . . . . 55

4.12 Electrolyte drive time-course spectral results . . . . . . . . . . . . 56

4.13 Quiescent yeast response time-course spectral results . . . . . . . . 57

4.14 Quiescent yeast drive time-course spectral results $\ldots \ldots \ldots$. . . . . 58

xii 
4.15 Active yeast response time-course spectral results $\ldots \ldots \ldots$. . . . . . 60

4.16 Active yeast drive time-course spectral results $\ldots \ldots \ldots$. . . . . . . 61

4.17 Quiescent yeast reference spectrum time-course results $\ldots \ldots$. . . . 63

4.18 Active yeast reference spectrum time-course results $\ldots \ldots \ldots 4$

4.19 Effect of boiling yeast . . . . . . . . . . . . . . . . 65

4.20 Effect of glucose on electrolyte . . . . . . . . . . . . . 67

4.21 Trial 1 response time-course spectral results $\ldots \ldots \ldots \ldots 72$

4.22 Trial 1 drive time-course spectral results $\ldots \ldots \ldots$. . . . . . 73

4.23 Trial 2 response time-course spectral results $\ldots \ldots \ldots \ldots$. . . . . . 74

4.24 Trial 2 drive time-course spectral results $\ldots \ldots \ldots \ldots 75$

4.25 Trial 3 response time-course spectral results $\ldots \ldots \ldots 76$

4.26 Trial 3 drive time-course spectral results $\ldots \ldots \ldots$. . . . . . . 77

4.27 Metabolic activity step function model . . . . . . . . . . . . . 82

4.28 Trial 3 time-course response fundamental $\ldots \ldots \ldots 5$

4.29 Trial 3 time-course drive current fundamental $\ldots \ldots$. . . . . . . 86

5.1 Warburg electrode equivalent circuit $\ldots \ldots \ldots . \ldots . \ldots 92$

5.2 DC interfacial impedance $\mathrm{I}-\mathrm{V}$ curve $\ldots \ldots \ldots 93$

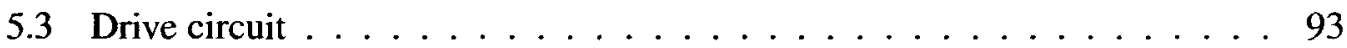

xiii 
5.4 Effect of electrode spacing $\ldots \ldots \ldots \ldots$

5.5 Quiescent yeast PSD . . . . . . . . . . . . . . . 102

5.6 Time-course normalized average power: Drive current signals . . . . . . . 106

5.7 Effect of air bubbles . . . . . . . . . . . . 108

6.1 Current compensation method output; Electrolyte, quiescent yeast and active yeast . . . . . . . . . . . . . . . . . 116

6.2 Polarization compensation method output; Electrolyte, quiescent yeast and

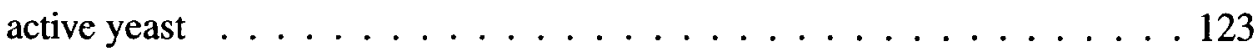




\section{List of Symbols}

\begin{tabular}{|c|c|}
\hline A & Amperes \\
\hline $\mathrm{AC}$ & alternating current \\
\hline $\mathrm{Ag}-\mathrm{AgCl}$ & silver-silver chloride \\
\hline ANN & artificial neural networks \\
\hline ATP & adenosine triphosphate \\
\hline $\mathrm{Au}$ & gold \\
\hline $\mathrm{C}$ & capacitor \\
\hline $\mathrm{Cm}$ & Coulomb metres \\
\hline $\mathrm{CO}^{2}$ & carbon dioxide \\
\hline DC & direct current \\
\hline $\mathbf{E}_{h c}$ & half-cell potential \\
\hline $\mathbf{F}$ & Farads \\
\hline $\mathbf{F}_{i n}$ & input frequency \\
\hline $\mathrm{Hz}$ & Hertz \\
\hline I & (effective) current \\
\hline $\mathrm{L}$ & litres \\
\hline mil & thousandths of an inch \\
\hline $\mathrm{mA}$ & milliamperes \\
\hline $\mathrm{mm}$ & millimetres \\
\hline mmol & millimols \\
\hline$\mu \mathbf{L}$ & microlitres \\
\hline $\mathrm{mV}$ & millivolts \\
\hline NIR & near-infrared \\
\hline NLDS & nonlinear dielectric spectroscopy \\
\hline nm & nanometres \\
\hline OGTT & oral glucose tolerance test \\
\hline$\Omega$ & Ohms \\
\hline
\end{tabular}




$\begin{array}{ll}\mathrm{pF} & \text { picofarads } \\ \mathrm{PLS} & \text { partial least squares } \\ \mathrm{PSD} & \text { power spectral density } \\ \mathrm{R} & \text { resistor } \\ R_{o} & \text { resistance between drive electrode pair } \\ R_{\text {rec }} & \text { resistance between recording electrode pair } \\ \rho & \text { resistivity } \\ \mathrm{S} & \text { Siemens } \\ \text { S. cerevisiae } & \text { Saccharomyces cerevisiae } \\ \mathrm{US}-\mathrm{FDA} & \text { United States Food and Drug Administration } \\ v_{s} & \text { voltage source } \\ \mathrm{Z} & \text { impedance } \\ \| & \text { in parallel with }\end{array}$




\section{Chapter 1}

\section{Introduction}

\subsection{Overview}

Diabetes mellitus is a chronic disease of the metabolic system with serious health implications for those suffering from the disease, and has considerable associated social costs. An important tool in the effective management of the disease is self-monitoring of blood glucose levels. Knowledge of blood glucose concentration allows the patient to understand how daily activities such as diet and activity affect their health, thus providing feedback in disease management. Additionally, knowledge of blood glucose concentration is required in implementing an insulin therapy regime.

The conventional device used in self-monitoring of blood glucose, based on the glucose oxidation reaction, is accurate, reliable and well proven. The drawback to this type of device is that a small blood sample is required in order to determine glucose concentration. This blood requirement limits the number of measurements that can taken throughout the day, due to discomfort caused from acquiring a blood sample and the associated risk of infection, as well as the cost of consumables. The drawbacks of this method which requires a blood sample provide strong motivation to develop a noninvasive glucose determination method, in which no blood sample is required. 
Much of the current research in noninvasive techniques for measuring blood glucose levels are typically optically-based, and work to identify glucose through some characteristic properties of the glucose molecule. These methods have exhibited poor specificity and low sensitivity for glucose [1], and will require considerable research efforts before sufficient accuracy is achieved [2]. Another approach that can be considered to measure blood glucose, rather than attempting to directly measure a glucose concentration, is to instead measure a physiological response to the presence of glucose. Physiological effects can include changes at a macro-level such as blood perfusion and temperature. Other effects occur at the cellular level, such as shifts in fluid and ions between the intracellular and extracellular environments. Indirect knowledge of glucose concentration through a physiological response could then be used to form a model to predict glucose levels based on the state of the known response. It is the latter approach that is considered in the body of this dissertation, in particular for a response occurring at the cellular level.

\subsection{Problem of Interest}

One of the benefits of predicting glucose concentration by measuring a correlated physiological response is that established sensing techniques can be considered. This avoids the complexity and cost associated with using state of the art sensing technology. The technique considered in this dissertation, nonlinear dielectric spectroscopy (NLDS), employs a conventional four electrode apparatus and has been shown in $[3,4,5]$ to be sensitive to the metabolic state of an organism. Since glucose is the primary fuel of cellular metabolic activity, NLDS is of interest in the noninvasive determination of blood glucose levels. NLDS measures the nonlinear response of a biological sample to a perturbating stimulus current. The relative magnitude of the spectral components of the response voltage has been shown 
to be correlated with metabolic activity [3].

The problem of interest in this dissertation is to determine if NLDS can be practically and effectively applied as a sensing modality in the noninvasive determination of glucose.

\subsection{Objectives and Goals}

The goal of the work presented in this thesis was to evaluate the practical aspects and confounding factors in the application of NLDS in a suspension of biological cells. The underlying motivation was to explore NLDS as a sensing approach for the noninvasive determination of blood glucose concentration.

The work of this thesis can be classified into four stages, with each stage having a set of objectives and goals. The work itself was iterative in nature. Stages were revisited and often interleaved in time to refine processes while working to achieve the ultimate goal.

The first stage of work was that of background literature research. The initial objectives in this stage were to understand the dielectric properties of biological cells and the properties of electrode-electrolyte systems, as well as the body of knowledge in NLDS. The initial goal of this stage was to have sufficient background to establish the requirements of the experimental apparatus as well as the data acquisition and signal processing software. As work on the other stages progressed, research became more focused on specific elements over a broader scope.

The second stage was the experimental work. The first objective of this stage was to establish a suitable experimental apparatus and data acquisition system based on the requirements formed in the background research or analysis stages. The second objective was to design and implement the experimental protocols to acquire data for control and correlation experiments. The goal of this work was to acquire data for analysis. 
The third stage of the work was the analysis phase. In this stage, one objective was to develop algorithms for data processing and analysis. The other objective was to form an equivalent circuit model to assist in analyzing the system. One goal of this work was to understand the effect of metabolically quiescent and metabolically active biological cells on the recorded data. The second goal was to understand the effect of system artifacts on the recorded data.

The fourth stage can be classified as the solutions stage. The objectives in this stage were to develop solutions to extract meaningful information from the data. In particular, this meant compensating for system artifacts imparted on the data to achieve the goal of revealing the true biological response.

As mentioned earlier the ultimate goal of this work is to provide an evaluation of NLDS in the context of the noninvasive determination of glucose. The following section outlines the contributions of this dissertation in NLDS.

\subsection{Contributions}

The first contribution that this thesis makes to the body of knowledge of NLDS is to propose a time-course analysis of spectral data and to show that it can be used in identifying a physiological response to glucose. This method illustrates the variability of the measurement over time, which previous methods do not. This in turn enables one to examine changes of metabolic state over time and ensures that observations are stable rather than occurring by chance. Data obtained with both gold and $\mathrm{Ag}-\mathrm{AgCl}$ electrodes for the electrolyte medium, and both the metabolically quiescent and metabolically active biological cell suspensions are examined using this method. Time-course data for $\mathrm{Ag}-\mathrm{AgCl}$ electrodes in NLDS of cell suspensions has not been previously presented. 
The second contribution is the application of a technique based on altering electrode spacing to analyze the electrode-electrolyte system. The technique used in this analysis is a variation on the electrode spacing method for measuring total electrode polarization as described in [6]. The application of this technique in this dissertation provides a means of examining the extent to which interfacial polarization is a function of effective current.

The third contribution of this thesis is the analysis of the disturbance imparted on the polarization layer by cellular bulk. While the possibility of such an effect was mentioned in [7] evidence was not provided.

The fourth contribution is the investigation and formation of a hypothesis regarding the current variation in the electrolyte, quiescent yeast, and active yeast cases. This work indicated that for an NLDS apparatus under voltage clamp conditions, changes in current can lead to systematic variation in polarization artifacts.

The fifth contribution of this thesis is the identification, analysis, and development of a compensation method to overcome a number of artifacts. The first use of this compensation method was to attempt to remove the artifacts resulting from the increase in effective current in the active case. The method was then developed further to compensate for electrode polarization artifacts. Part of this work was presented at the $28^{\text {th }}$ Annual International IEEE-EMBS Conference, Aug. 30 to Sept. 3, 2006, New York City, New York. and published in [8].

In light of these contributions, the conclusion can be made that future work is required in both the experimental apparatus and compensation techniques to reveal a biological signal that may be indicative of metabolic state. 


\subsection{Outline of the Dissertation}

Following this introduction, Chapter 2 provides background on diabetes and the importance of self-monitoring of blood glucose in the management of this disease. The technology used in conventional blood glucose monitors is first described, followed by an examination of the current research in noninvasive techniques for blood glucose determination.

Next, Chapter 3 presents the technique of nonlinear dielectric spectroscopy (NLDS) as a method to be considered in the noninvasive determination of blood glucose. In this chapter the necessary background related to the electrical properties of biological cells is first discussed. This is followed by further background information regarding a nonlinear phenomena of biological cellular behavior that NLDS leverages in classifying metabolic state. The fundamental work appearing in the literature in NLDS is then presented. Finally, the electrode-electrolyte sensor system used in NLDS and signal processing techniques are described.

With the necessary background provided, Chapter 4 discusses the primary experimental NLDS work undertaken in this thesis. First the experimental goals and test cases are described, followed by a description of the materials and methods. Three different experimental protocols and the results obtained are then examined. The first set of experiments attempts to repeat the work done by others, while the second and third sets of experimentation were done to implement improvements.

Chapter 5 presents the analysis of the experimental results. Particular attention is paid to artifacts arising in the system from electrode polarization effects and metabolic byproducts. With the analysis indicating that system artifacts can mask the biological signal of interest, the following Chapter 6 presents two software-based methods to compensate for system artifacts. The first method attempts to compensate for variation in conductivity 
brought about by metabolic byproducts, while the second method attempts to compensate for polarization artifacts.

Finally, the discussion presented in Chapter 7 summarizes the work of this dissertation, describes the key findings in the context of the goals of this thesis, and discusses directions for future work. 


\section{Chapter 2}

\section{Background}

\subsection{Overview}

This chapter presents background information on diabetes and methods used to detect glucose concentrations. The first section covers material related to diabetes and the role that blood glucose determination plays in disease management. The technology behind commercially available glucometers is also discussed here. The second section presents some current research into noninvasive methods of measuring glucose concentrations.

\subsection{Diabetes}

Diabetes mellitus is a chronic disease of the metabolic system affecting approximately 140 million people worldwide [1]. Complications arising from diabetes include stroke, cardiac diseases, high blood pressure, and blindness. The World Health Organization estimates that 1 in 20 deaths worldwide are attributable to diabetes.

Type 1 diabetes results from the body not being able to produce enough insulin to metabolize glucose sufficiently. Type 2 diabetes on the other hand results from the body being unable to use the insulin it produces to metabolize glucose. People with type 1 diabetes 
typically need to test blood glucose levels four times a day to determine insulin injection dosages for good control [9]. Those with type 2 diabetes generally do not require such active monitoring if they are not undergoing insulin therapy [9]. For patients undergoing insulin therapy frequent self-monitoring of blood glucose is the primary method in maintaining a healthy glucose level [9]. Patients with type 2 diabetes who are not medicating with insulin receive limited benefits from self-monitoring [10].

Glucose is the main source of energy within the body, and is carried in the blood to cells throughout the body. The levels of this carbohydrate in the blood will fluctuate throughout the day depending on activity and diet, among other factors. Insulin, a hormone produced in the pancreas, aids in this transport of glucose from the blood to the cells. A complete lack of insulin, or a reduced supply of insulin, or insulin that does not aid in effective glucose transport will result in cells being deprived of this energy source and a corresponding accumulation of glucose in the bloodstream. In the long term this can lead to blindness, cardiac disease, kidney failure, nerve damage and the loss of limbs among other pathologies.

According to [9], The Diabetes Control and Complications Trial, the results of which were published in 1993, studied patients who monitored blood glucose levels four times a day and responded with insulin therapy. Data from this study showed that patients undertaking this diabetes management approach of glucose monitoring had improved glycemic control and significantly reduced diabetes-related complications. Since the release of this data, self-monitoring of glucose levels has become the standard of care in managing diabetes.

The majority of glucometers used in self-monitoring are minimally invasive in that they require a minute blood sample. The site typically used for blood sample collection is the fingertip, although four devices have US-FDA approval for alternate site testing [9]. The 
basic operation of these devices is the same, regardless of where the sample is obtained. The skin is lanced to extract a drop of blood. The glucose concentration of the blood is then directly measured by the glucometer. The range of blood sample size for commercially available devices ranges from 0.3 to $10 \mu \mathrm{L}$ and test time ranges from 5 to 45 seconds [9]. As reported in [11] the US-FDA recommends devices have an accuracy of $20 \%$ or better, while the American Diabetes Association recommends $10 \%$ or better.

The principal on which conventional glucometers work is the glucose oxidation reaction, which is catalyzed by glucose oxidase. The products of the oxidation reaction, gluconic acid and hydrogen peroxide, are directly proportional to the glucose concentration. Potentiometry or amperometry is used to measure the products of the reaction. This method is well proven, and exhibits high sensitivity and selectivity [12].

While conventional glucometers are highly accurate and reliable, their requirement for a blood sample is a significant disadvantage. Discomfort from the lancing procedure, the risk of infection, and the risk of transmission of blood-borne diseases are factors motivating research into self-monitoring methods which do not require direct access to a blood sample. Methods which do not require direct access to blood or interstitial fluid will be referred to in this thesis as noninvasive. Noninvasive monitoring methods are also likely to be useful in continuous or near-continuous monitoring. This can be beneficial in providing higher resolution feedback to users about how their diet and activity levels affect their glucose levels throughout the day. This can help improve the disease management.

There is currently no noninvasive self-monitoring device approved for use by the USFDA. Despite the large amount of effort invested in researching noninvasive techniques, results have shown low sensitivity, low accuracy, and poor specificity [1].

The following section will present some of the noninvasive measurement techniques 
currently being researched. These are near-infrared absorption and scattering, Raman spectroscopy, photoacoustic, and polarimetry. A good review of noninvasive techniques related to glucose measurement can be found in [2]. A good report on noninvasive and minimally invasive optical techniques can be found in [13].

\subsection{Current Research in Noninvasive Methods}

\subsubsection{Near-Infrared Spectroscopy}

The optical technique of near-infrared (NIR) spectroscopy uses low intensity NIR light to illuminate the skin and then analyzes the properties of the light that is reflected or transmitted. Light is partially absorbed and scattered as it interacts with the tissue constituents. In the NIR range, spanning wavelengths from $750 \mathrm{~nm}$ to $2500 \mathrm{~nm}$, light is absorbed by water, fat, proteins and glucose [14]. The NIR range is of particular interest due to the lower water absorption as compared to the longer wavelength infrared light [15]. Analysis is done by measuring the change in received light intensity and applying multivariate calibration methods [12].

In the studies of [14], oral glucose tolerance tests (OGTT) were performed on three non-diabetic human subjects and glucose predictions were made using NIR reflectance spectroscopy. The best results obtained yielded a mean standard error of prediction of 1.41 $\mathrm{mmol} \cdot \mathrm{L}^{-1}$. These results were an improvement over the earlier work of others [14]. While this work has shown promise, considerable effort will be required to improve the calibration methods and repeatability [14].

In the studies of [16], oral glucose tolerance tests (OGTT) were performed on six subjects. Glucose predictions were made using NIR reflectance spectroscopy applied to the forearm, with the dermis tissue being optically probed. This work found a good correlation 
between the predicted and actual glucose values, but concluded that a larger number of test subjects was required to evaluate the final performance of the technique.

\subsubsection{Raman Spectroscopy}

Raman spectroscopy employs a monochromatic light source incident on the sample of interest. Most of the light is scattered elastically at the same wavelength. A portion of the light is scattered inelastically, with the photons gaining or losing energy due to transitions between oscillatory and rotational states of the scatterer molecules [17]. This inelastic scattering results in shifts in the wavelength of the scattered light. The resulting spectrum has distinct peaks which can be correlated with the chemical structure of the sample [13].

The advantage of Raman spectroscopy in biological tissue as compared to NIR spectroscopy is that the Raman spectrum of water is weak. This provides a good spectrum of the molecules contained in an aqueous solution with minimal interference from the large water volume [13]. The disadvantage with Raman spectroscopy is that received signals are weak, and are often below the fluorescence level of proteins [12]. With the advent of fast charge coupled device arrays (CCD) and high powered NIR lasers, the technology to perform research in this area has become more readily available [13]. The aqueous humor of the eye has been of interest in the measurement of glucose using Raman spectroscopy, due to the minimal background fluorescence in this tissue [13].

The study of [18] employed surface-enhanced Raman spectroscopy in which an optical window contacting the interstitial fluid was surgically implanted in a rat. Results of blood glucose predictions were relatively good with a root mean square error of calibration of $0.41 \mathrm{mmol} \cdot \mathrm{L}^{-1}$ and a root mean square error of prediction of $2.97 \mathrm{mmol} \cdot \mathrm{L}^{-1}$. While this approach requires the implantation of a sensor, further work may prove this technique 
beneficial in monitoring glucose levels in diabetics [18].

\subsubsection{Polarimetry}

Polarimetry, also an optical method, leverages the phenomenon that the linear polarization plane of light will rotate when passed through a sample. The rotation angle is proportional to the concentration of the substance under study [13]. For glucose, the polarization plane rotates to the right. In addition to the dependance of rotation angle on concentration, the path length of the light, as well as $\mathrm{pH}$ and temperature of the material also have an effect [13]. Polarimetry is a transmissive technique, as opposed to Raman and NIR spectroscopy, which are typically reflectance-based techniques.

The disadvantage of polarimetry in biological tissue is due to the high degree of scattering which occurs. This scattering effect can depolarize $95 \%$ of the transmitted light in a tissue sample $4 \mathrm{~mm}$ thick with an outer layer of skin [13]. The scattering problem severely limits the thickness of tissue with a skin layer that can be successfully probed. To avoid this problem, polarimetry has also been considered for measuring the glucose concentration of the aqueous humor. Motion artifacts due to eye movement and the rotational effect of the cornea have presented technical challenges that need to be overcome [13].

Work published in [19] presented a two wavelength polarimetry apparatus and motion compensation technique used in the in vitro measurement of glucose. This work has shown a considerable improvement in accuracy as compared to previous single wavelength techniques. Further work is still required to obtain successful in vivo results but the improvements presented here are a significant development in this method [19]. 


\subsubsection{Photacoustics}

Photoacoustic methods use an optical energy source such as a pulsed laser to irradiate a test substance. This absorption of the optical energy causes a rapid thermal expansion of the absorbing components, followed by a compression due to the inertia of the surrounding medium. This rapid physical deformation results in energy being released as an acoustic wave. The amplitude of the acoustic wave is linearly proportional to the absorbed energy density, while the shape of the waveform is defined by the distribution of the absorbing components, the properties of the light source, and boundary conditions in the substance [12]. In this method the transmitter is an optical source, while the receiver is a microphone.

Photoacoustic methods used in measuring glucose have shown higher detection sensitivity than the other optical absorption techniques. The technique is sensitive though to the confounding effects of temperature and pressure variation, as well as to interference from biological molecules [12].

The study of [20] employed a pulsed photoacoustic technique to measure a change in glucose concentration in pig's blood and Intralipid in vitro using two discrete wavelengths. In pig's blood the glucose-induced change in the peak-to-peak amplitude of the photoacoustic signal was $6 \%$ per $27.8 \mathrm{mmol} \cdot \mathrm{L}^{-1}$ increase in glucose concentration at $532 \mathrm{~nm}$, and $11.4 \%$ per $27.8 \mathrm{mmol} \cdot \mathrm{L}^{-1}$ at $1064 \mathrm{~nm}$. The increase using Intralipid was considerably smaller. This study showed the photoacoustic technique to be capable of detecting changes in glucose in in vitro experimentation. 


\subsection{Summary}

The monitoring of blood glucose concentrations in diabetics, particularly for those requiring insulin therapy, is vital in effective management of the disease. Conventional blood glucose monitors, based on the glucose oxidation reaction, are well established commercially and provide accurate, reliable results. The negative aspect of these conventional devices is that a small blood sample is required.

The motivation behind research into noninvasive glucose monitoring techniques is to find methods which do not require a blood sample, and can ultimately give results comparable to the conventional methods. Considerable effort has been applied to methods in working towards the goal of noninvasive monitoring. The four methods described earlier; NIR spectroscopy, Raman spectroscopy, polarimetry, and photoacoustics have shown promise. The main difficulty with these methods is that the glucose signature is weak compared to other chemicals in the skin and subcutaneous tissue. This results in low sensitivity and poor specificity for glucose [1].

The noninvasive methods presented earlier which are currently being researched attempt to measure the glucose concentration in a tissue sample. Another approach to monitoring glucose concentration is to instead measure a physiological response to glucose and correlate the response to glucose concentration. By measuring multiple physiological parameters and applying a data fusion model, it may be possible to predict the blood glucose concentration.

The advantage that the physiological glucose response approach provides is that a number of conventional sensing techniques can be applied. These could include electrocardiogram methods and bioimpedance methods, both of which are better established than the state of the art in optical methods. 
One such method that has appeared in the literature, and can be classified as a technique that measures a physiological response to glucose, is nonlinear dielectric spectroscopy. The application of this method is attractive as it can be incorporated into a conventional bioimpedance sensing apparatus, thus being well suited to a data fusion framework. Nonlinear dielectric spectroscopy will be discussed in detail in the following chapter to provide suitable background information on the technique and underlying biology. The experimental work and analysis of this dissertation will be focussed on evaluating nonlinear dielectric spectroscopy as an additional sensing modality in measuring a physiological response to glucose. 


\section{Chapter 3}

\section{Nonlinear Dielectric Spectroscopy}

\subsection{Overview}

The nonlinear response of biological cell suspensions to a sinusoidal perturbing field has been of interest as a potential method to probe the status of a biological system. Work appearing in the literature has suggested that this nonlinear response can indicate the metabolic state of a biological system $[3,4,5,21]$. This type of physiological information would be significant in applications such as the noninvasive determination of blood glucose concentrations.

Nonlinear dielectric spectroscopy (NLDS) measures the nonlinear response of a substance placed in a perturbating electrical field. Typically, a stimulating sinusoidal input signal is applied to a substance in the form of an $\mathrm{AC}$ voltage or current through a pair of drive electrodes. The test substance's response to this electric field is then measured as a voltage at a pair of recording electrodes. Unfortunately this measured response can also include measurement artifacts, such as those due to electrode polarization impedance. A single pair of electrodes can provide both stimulus and sensing capabilities, but typically a four electrode system is used to reduce electrode polarization artifacts in the recording pair [22]. 
In NLDS it is the nonlinear relationship between current and voltage that is of interest. In the time domain this nonlinearity appears as a distortion in the quasi-sinusoidal voltage at the recording electrodes, while in the frequency domain this distortion appears as distinct spectral components - the harmonics - occurring at integer multiples of the input frequency. Analysis is typically done in the frequency domain by examining the relative magnitude of the resulting harmonics.

This chapter will first examine the electrical properties of biological cells in general. This is followed by a study of the nonlinear electrical behavior of cells, and the relationship of this nonlinearity to glucose concentration. Thirdly, the fundamental NLDS work done by others is presented. The electrical properties of the electrode-electrolyte system are then examined to provide background information on system artifacts appearing in the recorded response. Finally, the signal processing and analysis methods used in NLDS are presented.

\subsection{Dielectric Theory Applicable to Biological Cells}

Biological material, including tissue and cell suspensions, can be described in terms of its dielectric properties: conductivity and permittivity. Conductivity is the proportionality factor between an electric field and current density. In biological matter, conductivity arises from ionic charge carriers and provides a measure of the ease with which ions move in the presence of an electric field. Conductance, denoted by $G$ (measured in Siemens S or $\mathrm{Ohms}^{-1}$ ), is given by

$$
G=\sigma \frac{A_{i}}{d_{i}}
$$


where $\sigma$ is the conductivity, $A_{i}$ is the cross-sectional area of the material, and $d_{i}$ is the length of the current path [23].

At low frequencies $(<0.1 \mathrm{MHz})$, conductivity occurs primarily in the electrolytic extracellular fluid. At higher frequencies cell membranes behave as a short circuit, resulting in the conductivity of the system approximating that of a suspension of nonconducting proteins in an electrolyte fluid [24].

The second material property, permittivity, is proportional to the ratio of the charge stored in a material and the electric field. This is a measure of the ability of local charges in a material to polarize in an electric field. In cellular suspensions the charges associated with permittivity are primarily those of the double layer of charge at the cell membrane, and those of dipolar molecules [25]. The double layer of charge arises from the electrochemical gradient between the intracellular and extracellular environments. In the yeast $S$. cerevisiae employed in the experimental work of this thesis, the electrochemical gradient is due to a concentration of negatively charged ions in the intracellular fluid, and a concentration of positively charged ions in the local extracellular fluid.

The equation describing the relationship between capacitance, denoted by $C$, and permittivity, ignoring field fringing effects, is

$$
\begin{aligned}
& C=\epsilon \frac{A_{c}}{d_{c}} \\
& C=\epsilon_{r} \epsilon_{0} \frac{A_{c}}{d_{c}}
\end{aligned}
$$

where $\epsilon_{r}$ is the relative permittivity of the material, $\epsilon_{0}$ is the permittivity of free space, $A_{c}$ is the area encompassing the charge, and $d_{c}$ is the separation between the double layer of charge [23].

Biological cellular material exhibits an out-of-phase loss factor, which can be described 
by the complex permittivity:

$$
\hat{\epsilon}=\epsilon^{\prime}-j \epsilon^{\prime \prime}
$$

where $\epsilon^{\prime}$ is the real part [23]. The imaginary part, denoted by $\epsilon^{\prime \prime}$ in [23], is

$$
\epsilon^{\prime \prime}=\frac{\sigma}{\epsilon_{0} \omega} .
$$

In (3.4), $\sigma$ denotes the conductivity, and $\omega$ denotes the angular frequency of the applied electromagnetic field. Conductivity $\sigma$ is a property of a material, and describes the mobility of charge carriers in the material in the presence of an externally applied electric field.

Permittivity and conductivity are both frequency dependant. In general, permittivity decreases with increasing frequency, and pioneering work by Schwan found this change occurred in three distinct steps known as the $\alpha, \beta$, and $\gamma$ dispersions [26]. The low frequency $\alpha$ dispersion, spanning milliHertz to kiloHertz, arises from ionic diffusion processes at the cell membrane. This dispersion exhibits a significantly large permittivity on the order of $10^{6} \mathrm{Fm}^{-1}$ to $10^{7} \mathrm{Fm}^{-1}$ in a suspension of cells, but the conductivity increase is only very slight at approximately $0.005 \mathrm{~S} / \mathrm{m}$ [24]. Despite the large permittivity values, the low frequencies here result in impedance being mainly resistive.

The radio frequency $\beta$ dispersion is found in the megaHertz range and results primarily from the charging of the capacitive double layer of the cell membrane. Permittivity values are typically in the range of $10^{3} \mathrm{Fm}^{-1}$ to $10^{4} \mathrm{Fm}^{-1}$ and the conductivity increase associated with this dispersion is approximately $0.4 \mathrm{~S} / \mathrm{m}[24]$.

Finally, the gigaHertz frequency range $\gamma$ dispersion arises from the dipolar relaxation of water molecules, which make up the greatest volume in soft tissues and cell suspensions. Permittivity values are typically in the range of $10^{2} \mathrm{Fm}^{-1}$ while the conductivity increase associated with this dispersion is around $70 \mathrm{~S} / \mathrm{m} \mathrm{[24].}$ 


\subsection{Biological Response}

Glucose is the primary source of fuel in living organisms. The breakdown of glucose into adenosine triphosphate (ATP) provides energy in a form that can be used by cells. It is this breakdown, or catabolism of glucose that is referred to as metabolic activity in this thesis. In searching for a biological response to glucose that can be used to make predictions about glucose concentration, one possibility is the electro-chemical behavior at the cell membrane through which nutrients and waste must pass. This movement of material occurs as part of the metabolic process. As described in Section 3.2, an applied electric field yields membrane effects in the $\alpha$ and $\beta$ dispersions. In particular, effects associated with ionic diffusion processes through the cell membrane are found in the low frequency $\alpha$ dispersion [24]. Thus a low frequency electric field applied to a biological sample could yield information about the movement of ions through the cell membrane, and both theoretical and experimental work has shown this to be the case $[27,3]$.

\subsubsection{Transport Enzymes}

To maintain homeostasis, materials must be able to pass through the plasma membrane in a controlled manner. The movement of materials through the plasma membrane can be simply described as the uptake of nutrients and the expulsion of wastes. Two processes enable this transport. The first process is a passive movement along an electrochemical gradient, while the other process is an active transport, or pumping against an electrochemical gradient. It is this active transport mechanism, specifically the $\mathrm{H}^{+}$ATPase proton pump, that is significant in the nonlinear phenomenon examined in the NLDS work of this thesis.

$\mathrm{H}^{+}$ATPase is a proton pump used to control intracellular $\mathrm{pH}$, and to create an electri- 
cal gradient to enable the uptake of nutrient ions such as $\mathrm{K}^{+}$[28]. The proton pump does this by binding $\mathrm{H}^{+}$on its intracellular side, then changing conformation or shape to physically transport the substance to the exterior. This transport mechanism can be described in general by two extreme conformation states, denoted by $E_{1}$ and $E_{2}$ respectively. Each state exhibits a different affinity for the transport ion, to enable binding and release. The yeast plasma membrane $\mathrm{H}^{+}$ATPase transport mechanism is similar to that of other fungi ATPases, as well as mammalian ATPases [29].

$\mathrm{H}^{+}$ATPase is a large dipolar molecule fixed in the plasma membrane. In the presence of an applied static electric field, a dipolar element will exhibit an attractive force on one pole, and a repulsive force on the other pole. If the dipole element is free to move, it will rotate in response to these attractive and repulsive forces to align its dipole moment with the applied electric field. In the presence of an applied AC electric field, the same dipolar element would oscillate, also to align its dipole moment with the oscillating applied electric field. The dipolar structure of the $\mathrm{H}^{+}$ATPase protein causes it to experience an oscillating force in the presence of an applied AC electric field. Because this protein is fixed in the cell membrane, it is unable to rotate to align its dipole moment with the applied field. Instead of rotating, it can change conformation to reduce the forces acting on it. Therefore, an applied AC field can cause the protein to oscillate between conformations, resulting in the transport of ions [30]. The time delay of the protein conformation changes due to frictional and other forces acting on the protein results in a nonlinear distortion of the electric field, which is observed at the recording electrodes.

Two distinct active transport energy sources can be defined.

1. In the metabolically quiescent case, active transport energy is supplied by an applied oscillating electrical field. 
2. In the metabolically active case, active transport energy is supplied by the breakdown of glucose into ATP.

In the metabolically quiescent case, the applied electric field causes the protein to do work, resulting in a characteristic distortion of the applied electric field $[3,27]$. In the metabolically active case, the ATP driven conformation changes result in another characteristic distortion of the applied electric field [3,27].

The relatively small magnitude of dipole moment of the $\mathrm{H}^{+}$ATPase protein would be difficult to observe at the record electrodes without the amplification effect of the cell membrane. The dipole moment of a typical protein is on the order of 100 to 400 debye $\left(3.34 \times 10^{-27}\right.$ to $\left.13.3 \times 10^{-27} \mathrm{Cm}\right)[30]$, a magnitude that would appear insignificant relative to the strength of applied fields typically employed in bioimpedance work ( 0.1 to 5 $\mathrm{V} \cdot \mathrm{cm}^{-1}$ ) [3]. The cell membrane provides an amplification of the electric field at the transmembrane protein that is dependant on cell size [30]. S. cerevisiae, being an approximately spherical cell 5 to $10 \mu \mathrm{m}$ in diameter, would cause the $\mathrm{H}^{+}$ATPase protein to experience a gain of approximately 1000 [30]. Thus the electrical response of a transmembrane protein can have an observable effect on an applied electrical field.

The theoretical development of a nonlinear response indicative of transmembrane protein state shows the magnitude of the second harmonic to be sensitive to substrate levels [27]. Experimental work, first presented by Woodward and Kell in [3], found that in a suspension of the yeast $S$. cerevisiae a repeatable harmonic pattern was observed in the presence of glucose, and a different harmonic pattern in the absence of glucose. The yeast suspension is defined as being in a quiescent state when there is no metabolizable energy source available. According to [3], this quiescent state is characterized by dominant odd harmonics. The yeast suspension is defined as being in an active state when a metaboliz- 
able source, such as glucose, is supplied to the yeast. According to [3], this active state is characterized by dominant even harmonics. The a priori metabolic state of yeast can then be determined by examining the behavior of the second and third harmonic magnitudes.

\subsection{Fundamental Work in NLDS}

In the application of NLDS, a stimulating sinusoidal input signal is typically applied to a substance in the form of an $\mathrm{AC}$ voltage or current through a pair of drive electrodes immersed in a suspension of cells. The suspension's response to this electric field is then measured as a voltage at a pair of recording electrodes. The standard four electrode configuration, as shown in Fig. 3.1, is typically employed, with the outer electrodes supplying the stimulus signal and the inner electrodes sensing the response.

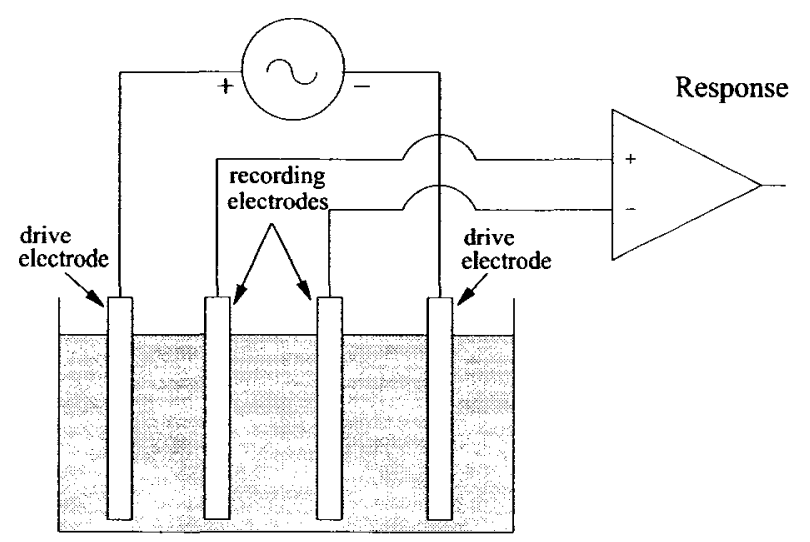

Figure 3.1: Four electrode NLDS apparatus: The four electrode NLDS apparatus injects current through the outer drive electrodes, while the inner recording electrodes measure the response voltage. 


\subsubsection{Voltage Clamp Work}

In this section, the pioneering NLDS work of [3] is described. This NLDS apparatus used a low frequency sinusoidal stimulus under voltage clamp conditions. The voltage at the stimulus electrodes was fixed, and the effective current supplied to the system was allowed to float in response to the system. To separate the biological response from system artifacts, a reference spectrum method was employed, requiring the spectrum of:

- the reference medium, consisting of a $0.1 \%$ salt solution in distilled water; and

- the test medium, consisting of a suspension of the yeast $S$. cereviaiae rehydrated in the reference medium.

The power spectral density (PSD) estimate was obtained for these two mediums using Welch's method with a window size of 512 samples, recorded at $500 \mathrm{~Hz}$ for a period of time spanning 10 windows. Data were gathered in parallel, and the conductivity of the reference medium was adjusted by adding distilled water to maintain the equality of conductivity for both the reference and test mediums.

The reference spectrum method used in [3] attempts to separate the nonlinearities due to the biological cells from the nonlinearities due to the electrode-electrolyte system. This extraction of the biological signal is done by subtracting the magnitude in $\mathrm{dBs}$ of the reference PSD from that of the test PSD. This method is not mathematically correct in that a linear operation is performed on a nonlinear system and does not isolate the true biological response from system artifacts [7]. However, the results presented in [3] indicate a repeatable harmonic signature which differs between the metabolically quiescent and active cases. 
The results of the reference spectrum method provide some useful information, which is described in the following list [3].

- The addition of yeast to the reference solution, referred to as the quiescent state, is characterized by a significant negative third harmonic, whose magnitude is related to the concentration of yeast cells, and small positive seventh harmonic. Destructive interference between the biologically generated third harmonic and nonlinearities in the signal source is stated as the cause of the negative sign of the third harmonic, but no evidence to confirm this is given.

- Boiling the quiescent yeast sample kills the cells. No harmonics are generated in this case.

- The biologically generated third harmonic has a significant magnitude only within specific frequency and voltage windows. For the applied voltage this window is between 0.6 and $2.1 \mathrm{~V}$. The source frequency window is maximal between 15 and 20 $\mathrm{Hz}$.

- The addition of glucose to the quiescent case transforms the yeast sample to the active state. This active state is characterized by increased second and fourth harmonics, with the odd harmonics of the quiescent case disappearing.

- As a control experiment, sodium metavanadate was added to the yeast suspensions. The activity of the $\mathrm{H}^{+}$ATPase enzyme is inhibited in the presence of sodium metavanadate. The purpose of this control was to determine if the nonlinear signal observed was due to $\mathrm{H}^{+}$ATPase enzyme or some other effect. The addition of vanadate to both the quiescent and active cases effectively prevented any nonlinear response. 
Time course data is not presented in [3], so the stability of any harmonic patterns is unknown.

\subsubsection{Current Clamp Work}

Rather than using a sinusoidal voltage source as the perturbating signal, the NLDS apparatus of [7] employs a sinusoidal current source. The stated advantage of this system is that any nonlinear drive electrode effects are minimized. The effective current in the region between the inner voltage sensing electrodes will therefore be sinusoidal.

Using a biological sample and electrolyte similar to those of [3], a constant current source apparatus with platinum electrodes produced results in which the harmonic magnitude varied greatly over an extended period [7]. Time-course data was not provided. This initial work of [7] could not repeat the results of [3], and no significant repeatable harmonics were observed. It was found that the addition of yeast to the electrolyte medium increased the nonlinearity of the system, but that the harmonic magnitudes exhibited substantial instability. It is not surprising that this work failed to repeat the results of [3] as a considerably different apparatus was used [31].

Work presented in [7], and extended in [32] suggested that harmonics observed in [3] were the result of cells modifying the electrode interface, but qualitative evidence was lacking. The authors of [31] countered this argument by reiterating that the control experiments in [3] strongly support the theory of a nonlinear response due to the $\mathrm{H}^{+}$ATPase enzyme. Furthermore, [5] suggested that the current clamp approach is not suitable in NLDS as it "forces an apparent linearity on the system". 


\subsection{Electrode-Electrolyte System}

The electrode-electrolyte component of the NLDS system is also a significant source of the nonlinearity measured at the voltage sensing electrodes.

The function of the electrodes can be twofold. The recording electrodes are used to measure the potential difference across them, which is quantified by some associated electronic circuit. For the recording electrodes the associated electronics are responsible for amplification, filtering and digital sampling. The role of the drive, or current injecting electrodes, is to conduct a current across the interface between the electrolyte solution and the associated electronic circuit. With the drive electrodes, the associated electronics provides the perturbating AC signal. Current flow in the electrolyte is ionic, while current flow in the electronic circuits is due to the movement of electrons. Thus the electrode must act as a transducer between these two types of charge carriers. The mechanism which enables this transduction is an oxidation-reduction (redox) chemical reaction. When current flows from electrode to electrolyte oxidation reactions are dominant. Reduction reactions are dominant when current flow is from electrolyte to electrode.

The immersion of an electrode into an electrolyte solution containing ions of the electrode starts a redox reaction at the interface. This redox reaction causes a local redistribution of charge until equilibrium is established. The resulting double layer of charge at the interface causes this region to be at a different potential than the bulk of the solution. This potential difference is known as the half-cell potential.

Electrode-electrolyte systems under low current conditions are typically treated as linear. High resolution measuring equipment can determine nonlinear electrode behavior at extremely low stimulating amplitudes [33]. This nonlinearity increases with current, and the level at which it becomes significant depends on the physical properties of the system, 
such as electrode material and electrolyte concentration [24]. The mechanism which causes this nonlinearity is electrode polarization. The polarization potential can be categorized by three basic overpotential mechanisms resulting in impedances within the system [34].

\subsubsection{Electrode Polarization}

The first polarization mechanism is the ohmic overpotential, which arises from the impedance of the electrolyte. This overpotential is the voltage drop along the current path in the electrolyte solution. The resistive component of electrolyte impedance can be a function of frequency and current, resulting in a nonlinear current-voltage relationship. This is particularly true in weak electrolytes [34] and in the presence of high electric fields [35].

The second mechanism contributing to the polarization potential is the concentration overpotential, which is caused by the redistribution of ions at the electrode-electrolyte interface under current flow conditions. This redistribution of ions causes the half-cell potential to be modified from its equilibrium state. Again, this impedance can be a function of current and therefore give rise to nonlinearities in the system [36, 37].

Finally, the activation overpotential is the result of the activation energy barrier that must be overcome for the redox reaction at the electrode interface to proceed. This barrier differs between oxidation and reduction processes, and therefore between anode and cathode as well.

When treated as linear, these three overpotentials are summed as impedances in series to give the polarization potential. Electrode polarization effects are proportional to drive current and electrode surface area, and at the current densities employed in NLDS, nonlinear electrode behavior can be significant [5]. The polarization layer is also unstable, which can result in spurious nonlinearities which from range from DC to $100 \mathrm{~Hz}$ [5]. 


\subsubsection{Polarizable and Nonpolarizable Electrodes}

In theory, electrodes can be placed in two ideal categories: perfectly nonpolarizable and perfectly polarizable.

Perfectly nonpolarizable electrodes are those in which current flow across the interface has no energy barrier to overcome. The advantage of this type of electrode in NLDS is that polarization effects at the interface are reduced. However, the tradeoff is that the redox reaction occurring at the interface alters the composition of the electrode over time, which can introduce other artifacts into the system. A commonly used example which approaches this ideal is the $\mathrm{Ag}-\mathrm{AgCl}$ electrode.

Perfectly polarizable electrodes, on the other hand, allow no actual charge to cross the interface, and all current is displacement current. In this case, the double layer of charge at the interface acts as a capacitor, and 3.2 applies. These types of electrodes exhibit a strong concentration overpotential [34]. The relatively inert nature of the metals that are considered to approach perfectly polarizable electrodes is advantageous because the electrode composition changes little over time. Examples of this type of electrode are platinum and gold electrodes.

\subsubsection{Electrode Types in NLDS}

In NLDS, electrodes of both polarizable and nonpolarizable types have been employed. Woodward and Kell [5] found gold electrodes to be the most stable in terms of harmonic magnitude, followed closely by $\mathrm{Ag}-\mathrm{AgCl}$. Their work also found the overall harmonic signature measured at the record electrodes in the $\mathrm{Ag}-\mathrm{AgCl}$ case to be reduced compared to the gold electrodes. The NLDS apparatus of [21] employed copper electrodes for both drive and sensing duties. A high-sensitivity magnetic field detector was also employed in 
[21] in place of the recording electrodes, providing the advantage of avoiding polarization effects that can appear in the record electrodes due to current leakage or electrode fouling.

Interestingly, lower linear impedance due to interfacial polarization does not necessarily mean that polarization harmonics are reduced as compared to electrodes with a higher linear impedance [5]. The example given is that platinum electrodes exhibit greater polarization harmonics than gold despite the lower polarization impedance. Platinum electrodes were used in the work presented in [7], in which a significant variation in harmonic magnitude was observed.

\subsubsection{Electrode Nonlinear Artifact Minimization}

Electrode polarization effects at the low frequencies used in NLDS of biological cells are a significant hindrance. The electrode-electrolyte impedances and those of the biological cells appear in the system as impedances in series, and as such, separating their respective nonlinear contributions to the measured response is difficult [38]. When measuring the response of the cells, confounding nonlinearities that may mask the biological signal of interest are introduced. Minimizing electrode polarization effects is therefore important. Hardware approaches to reduce electrode polarization effects typically include polishing electrode surfaces. The fractal nature of the electrode surface may affect polarization and is therefore minimized by polishing regimens [5]. Polishing can also remove surface contaminants which modify electrode polarization. A polymer electrode coating has shown promise in preventing electrode fouling and extending operating time before recleaning is required [39]. 


\subsection{Signal Analysis and Processing}

Signal analysis and processing techniques in NLDS can be divided into two classes. The first class includes techniques used to minimize system artifacts, thus emphasizing the biological response. The second class includes signal analysis techniques that are predictive in nature, and provide information about the metabolic state of the biological cells and glucose concentration from the measured response.

Fundamental to both classes of signal processing is the transformation of data from the time domain to the frequency domain. The frequency domain representation, or spectrum, reveals the harmonic components of the signal. Nonparametric transform methods using a fast Fourier transform (FFT) algorithm are typically used. Welch's method of timeaveraged modified periodograms with a Blackman window was used in [3] to produce an estimate of the power spectral density (PSD). Using a different windowing function, overlap, number of samples per data block, and/or the number of blocks can affect the PSD [40] and can therefore affect interpretation of relative harmonic magnitude.

The first class of signal processing to be discussed is the minimization, or separation of polarization and other electrode-electrolyte system artifacts from the biological signal. The reference spectrum method used in [3], in which the harmonic magnitudes of the reference spectrum are subtracted from the corresponding harmonic magnitudes of the biological sample, was described previously in Section 3.4.1. Work presented in [41] precluded the need for reference spectrum data by using principal components analysis (PCA) to identify systematic variations in the signal. This PCA approach treats artifacts as random noise.

Schwan has performed extensive work in the area of electrode polarization and the associated nonlinear phenomena $[36,42,38]$. Schwan's presentation of Sauer's nonlinear voltage divider [38] provided a treatment for modelling the nonlinear state of an electrode- 
tissue system. The nonlinear voltage divider separated the system into two series impedances:

1. a nonlinear impedance representing the electrode-tissue interface; and

2. a linear impedance representing the tissue impedance.

While the nonlinear voltage divider provides a means for modelling and separating the polarization nonlinearities from the biological impedance, it is not applicable to NLDS since a nonlinear tissue impedance is of interest. As mentioned earlier, separating the nonlinear contribution of electrode polarization from that of the biological cells from the recorded signal is difficult [38] and is a significant obstacle in overcoming the difficulties of NLDS [43].

The second class of signal processing and analysis is the prediction of glucose concentration using the nonlinear biological response. The authors in [5] applied partial least squares regression (PLS) analysis to yeast samples in vitro, sheep's blood in vitro, and a human subject in vivo, and also applied artificial neural networks (ANN) analysis to yeast samples in vitro. Neural network analysis with yeast samples was found to give similar predictions for both the reference spectrum method (the difference in magnitudes between the reference and the active yeast sample) and for the active yeast spectrum. This method appears to work well for nonlinear systems, and the published plots show a reasonable prediction of glucose concentrations for the active yeast case for data from within the same set.

PLS analysis was also performed on active yeast samples in [5]. This work also showed reasonable predictions of glucose concentration with good results within a data set, and the ability to predict trends amongst data sets. Tests were also conducted using sheep's blood in 
vitro, and modifying the glucose concentration. This sheep's blood work found a better prediction occurred in the presence of erythrocytes (red blood cells), but was unable to predict glucose concentration when erythrocytes were absent. The erythrocyte enzyme responsible for this result was not determined. Finally, PLS analysis was done on data obtained noninvasively from the forearm of a live human subject. Gold electrodes were fastened to the skin, and an oral glucose tolerance test was done. Blood glucose was measured using a Reflolux blood glucose monitor. Predictions were not as good as those obtained with the yeast samples and with the sheep's blood, exhibiting considerable noise and poor repeatability.

From the work of [5] it can be concluded that if electrode polarization artifacts remain stable, which is more likely within a data set, a reasonable prediction of glucose concentration can be made using NLDS. Secondly, the reference spectrum method when combined with PLS or ANN does not provide any benefit over analysis of the sample spectrum alone. This is significant in that an NLDS apparatus can be simplified and experimental protocols made more flexible and better suited to noninvasive blood glucose determination if the reference spectrum can be neglected.

\subsection{Summary}

This chapter examined the fundamental work in NLDS and provided the necessary background information on biological systems, electrode-electrolyte systems and data analysis techniques related to NLDS.

This background research found that there is a theoretical development relating a nonlinear biological response to metabolic activity. Following up on this theory, the experimental work of others used a relative change in harmonic magnitude as an indicator of metabolic state. This observation though was questioned by other researchers. 


\section{Chapter 4}

\section{NLDS Experimental Work}

\subsection{Overview}

The initial experimental work in this thesis was undertaken with the goal of repeating part of the work of completed by Woodward and Kell, which was presented in their first paper on NLDS of yeast cells [3], and ultimately to evaluate the technique of NLDS as a possible method for the noninvasive determination of blood glucose concentration. Thus the methods and materials employed in the first set of experiments are closely aligned with those found in [3]. Improvements were made to the experimental apparatus and protocol, and a second and third set of experiments were undertaken.

The following list highlights some of the questions that the experimental work of this thesis endeavors to answer.

- Does the addition of the yeast $S$. cerevisiae to an electrolyte medium affect the relative magnitude of the harmonics?

- Does the addition of glucose to a quiescent suspension of $S$. cerevisiae affect the relative magnitude of the harmonics?

- Does the addition of glucose to an electrolyte solution affect the relative magnitude 
of the harmonics?

- Do nonlinear effects differ between living and dead cells?

- Are nonlinear effects due to biological responses, or are system artifacts masking the biological signal?

- Furthermore, are these nonlinear effects repeatable, and do they match the results published in [3]?

\subsection{Materials and Methods}

\subsubsection{Apparatus}

A four electrode system was employed as shown in Fig.4.1 under voltage clamp conditions, with the outer two electrodes supplying the perturbating current and the inner two electrodes recording the response voltage. Electrodes were gold plated pins with a square cross-section measuring $0.64 \mathrm{~mm}$ by $0.64 \mathrm{~mm}$, and a total length of $14 \mathrm{~mm}$. Electrodes were spaced apart $2.54 \mathrm{~mm}$ on-center and were immersed in the test sample to a depth of 8 $\mathrm{mm}$.

The sinusoidal drive input at $1.5 \mathrm{~V}$ was supplied by a function generator (Agilent 33120A, Agilent Technologies, Palo Alto, California). Characterization of this function generator with a resistive load indicated that harmonic content was more than $75 \mathrm{~dB}$ below the fundamental. In [3], the authors used an input sinusoid at $20 \mathrm{~Hz}$, but in the experimental work of this dissertation the input was set at 16 or $17 \mathrm{~Hz}$ to prevent harmonics from falling on $60 \mathrm{~Hz}$ power mains noise. This choice of input frequency precluded the need to filter power mains noise, and allowed analysis to ignore any spectral components at 60 $\mathrm{Hz}$. Drive current was monitored by sampling the voltage across a $470 \Omega$ resistor $R_{m}$, in 


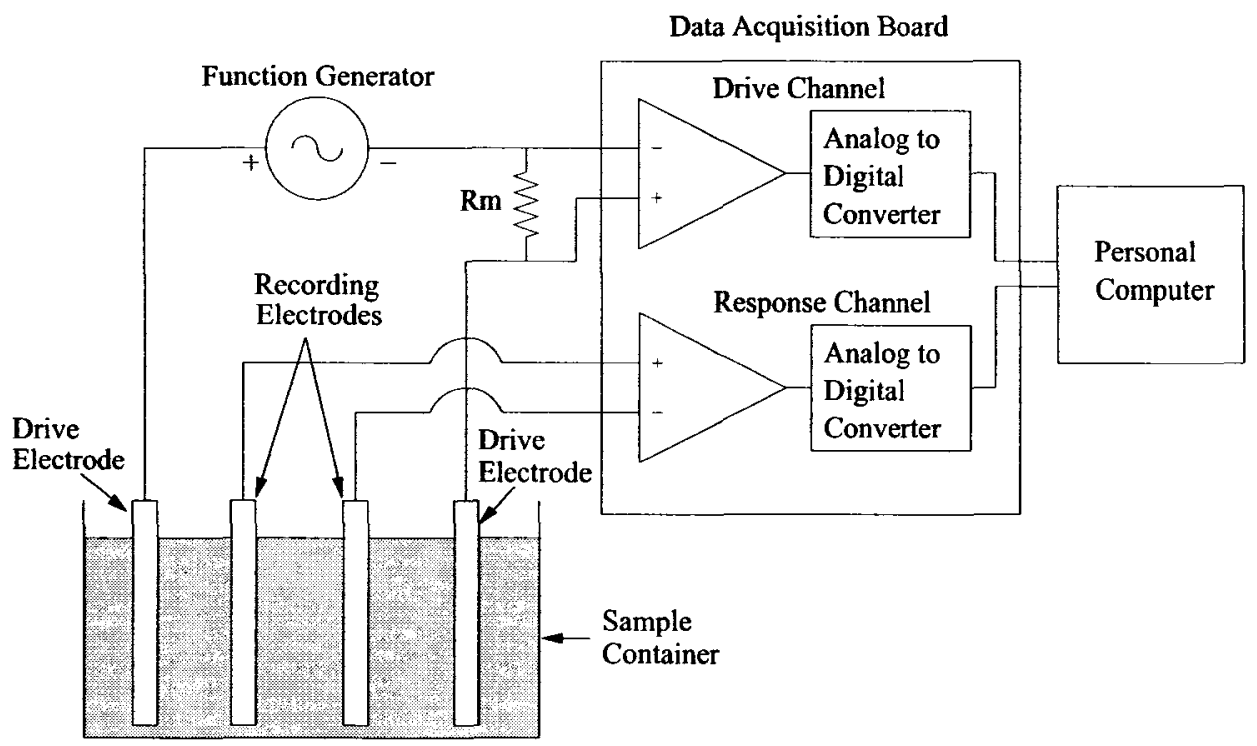

Figure 4.1: NLDS experimental apparatus.

series with the signal source. The value of $R_{m}$ was chosen to limit current in the range of 1 $\mathrm{mA}$ to prevent electrolysis. This current monitoring resistor $R_{m}$ was a novel addition to the NLDS apparatus, and was not found in the apparatus of others. The system response was measured by sampling the voltage across the inner recording electrodes. Amplification, sampling and analog to digital conversion were done using a multi-channel data acquisition board (National Instruments PCI 6023E, National Instruments Corp., Austin, Texas) with 12 bit resolution, operating at a sampling rate of $2048 \mathrm{~Hz}$, and controlled through a PC running LabVIEW software (LabVIEW 7.2, National Instruments Corp., Austin, Texas).

Apparatus characterization revealed that concurrent sampling of the drive and response channels with this hardware resulted in crosstalk noise between channels. This prohibited the interleaving of channel samples, so data in this initial work were collected for one channel at a time. This limitation prevented the comparison of the drive and response signals at the same point in time. 


\subsubsection{Medium and Cell Suspension}

Biological suspensions were prepared in an electrolyte medium composed of $20 \mathrm{mmol} \cdot \mathrm{L}^{-1}$ of $\mathrm{KH}_{2} \mathrm{PO}_{4}, 30 \mathrm{mmol} \cdot \mathrm{L}^{-1}$ of $\mathrm{KCl}$, and $20 \mathrm{mmol} \cdot \mathrm{L}^{-1}$ of $\mathrm{MgCl}_{2}$ in distilled water, as given in [3]. The biological cells were the yeast $S$. cerevisiae, rehydrated in the medium from a

freeze-dried powder (Muntons Active Brewing Yeast) at a concentration of $50 \mathrm{mg} \cdot \mathrm{mL}^{-1}$, and allowed to stand for one hour. The quiescent case has no metabolizable substrate added to the yeast. In the active case, glucose at a concentration of $170 \mathrm{mmol} \cdot \mathrm{L}^{-1}$ is dissolved in the medium and mixed in with the yeast. The reference case consist of only the electrolyte medium. The sample container was a $50 \mathrm{~mL}$ glass beaker with a diameter of $30 \mathrm{~mm}$. Sample sizes of $30 \mathrm{~mL}$ were pipetted into the sample container.

\subsubsection{Signal Processing}

Signal processing was performed on a PC running MATLAB software (MATLAB 7.0.4, The Mathworks Inc., Natick, Massachusetts). Frequency domain analysis was done by estimating the power spectral density using Welch's averaged, modified periodogram method. Signals were windowed with a Hamming window of length 1024 samples and overlapped by $50 \%$.

\subsection{Experimental Test Cases}

To answer the questions posed in Section 4.1, a number of different experiments were undertaken in which the nonlinear behavior of the system under test was examined. These test cases are described in the following subsections. 


\subsubsection{Electrolyte Medium}

The electrolyte medium provides a suitable environment in which yeast can be either metabolically quiescent or active. It does this by providing the necessary ingredients to synthesize ATP, the compound catalyzed by ATPase to release energy into the cell. The ions of this salt solution are also the charge carriers responsible for current flow through the test sample.

The spectrum of the medium provides a reference baseline, and can be a means of gauging the extent to which the electrode-electrolyte system produces artifacts in the biological response. The data were required in order to perform the reference spectrum analysis of [3].

This test was performed by pipetting the electrolyte medium into the sample container and recording the response and current monitoring channels.

\subsubsection{Quiescent Yeast Suspension}

The purpose of this test case is to acquire the spectrum of a metabolically inactive suspension of $S$. cerevisiae under the influence of the perturbating AC field. This spectrum will yield the harmonic signature of the transmembrane protein $\mathrm{H}^{+}$ATPase as it oscillates between the conformation states $E_{1}$ and $E_{2}$ in response to the applied electric field. The measured response also includes the system artifacts.

This test was performed by adding freeze-dried $S$. cerevisiae cells to the electrolyte medium. The cells were allowed to rehydrate for twenty minutes, after which the suspension was stirred. 


\subsubsection{Active Yeast Suspension}

The purpose of this test case is to acquire the spectrum of a metabolically active suspension of $S$. cerevisiae under the influence of the same perturbating AC field of the previous two test cases. The response signal will yield the harmonic signature of the transmembrane protein $\mathrm{H}^{+}$ATPase as it actively pumps hydrogen ions from the intracellular fluid while metabolic activity occurs.

This test was performed by stirring in glucose at a concentration of $170 \mathrm{mmol} \cdot \mathrm{L}^{-1}$ to the quiescent yeast suspension of the previous test case. S. cerevisiae is a top-fermenting yeast, meaning that when metabolically active, the cells rise to the solution surface. Thus after the initial stirring this suspension was left undisturbed for all data sets as the yeast tended to re-suspend with metabolic activity.

\subsubsection{Boiled Active Yeast Suspension}

This test case was performed by gently boiling the active case suspension for three minutes, allowing the suspension to cool, and then adding distilled water to replace that lost through evaporation. The purpose of this test case is to determine if a suspension of dead cells exhibits the same properties as a live suspension.

\subsubsection{Electrolyte Medium and Glucose}

This test case was undertaken to examine the response of the electrolyte solution with the addition of $170 \mathrm{mmol} \cdot \mathrm{L}^{-1}$ of glucose; the same glucose concentration used in the active yeast case.

This test was performed by pipetting the electrolyte medium into the test cell, and 
stirring in glucose at a concentration of $170 \mathrm{mmol} \cdot \mathrm{L}^{-1}$.

\subsection{Sequential Experimental Work}

This section presents the first experimental NLDS results in which data for each test case were collected sequentially. The details of the experimental materials and methods used in this first work are described in Section 4.2. The goal of this initial experimentation was to acquire data under similar conditions to those of [3], and to compare results.

\subsubsection{Experimental Protocol}

Data for each of the different test cases were collected under similar conditions at approximately $22^{\circ} \mathrm{C}$ using the same sample sizes for three experimental runs. Experimental runs took place over several days. Within a test case, one data set consists of six consecutive two minute trials. Data sets for the drive and response channels were acquired consecutively. Therefore a data set for both the drive and response channels spans approximately 24 minutes. This allowed recording two data sets for each channel every hour, with 12 minutes available for equipment set-up and adjustment. The total duration of each test case varied depending on the test case goal. Experiments with no biological matter were sampled over a one hour time period, while the more dynamic biological systems were sampled over a six hour period.

New electrodes were used for each data set in order to reduce electrode fouling, and the solution or suspension and electrodes were left undisturbed for the total 12 minute duration.

Signal processing was done by windowing each two minute data record into one second segments to correspond with the method of [3]. The PSD of each segment was then calculated and the average PSD over ten seconds was plotted, also as in [3] . Error bars on the 
following plots indicate three standard deviations of the harmonic magnitudes calculated over the two minute data record.

\subsubsection{Sequential Experimental Results}

\section{Electrolyte Medium}

The PSD plot of the electrolyte solution measured at the response electrodes is shown in Fig. 4.2. As shown by the error bars, the fundamental and the fourth harmonic exhibit reasonably stable behavior. All other spectral components showed considerable instability. The primary feature of interest in [3] is the relative magnitude of the second and third harmonics, for which the electrolyte test case was characterized by a third harmonic greater than the second. Again, as shown by the error bars on the second and third harmonics, over a two minute data record, periods in which the second harmonic was greater than the third were observed. Periods in which the third harmonic was greater than the second were also observed in some of the repetitions. The spectral component occurring at $60 \mathrm{~Hz}$ was attributed to power mains noise and was ignored in the analysis.

The plot of Fig. 4.3 shows the spectral components of data collected across the current monitoring resistor $R_{m}$. The harmonics observed in the drive signal indicate that effective current in the system is nonlinear. The harmonic magnitudes also display instability, as the shown by the error bars. The instability in the fundamental indicates that the system is transient and has not settled to an equilibrium point. Since the response and drive signals were not recorded simultaneously, correlating the transient behavior between the two signals is not feasible. 


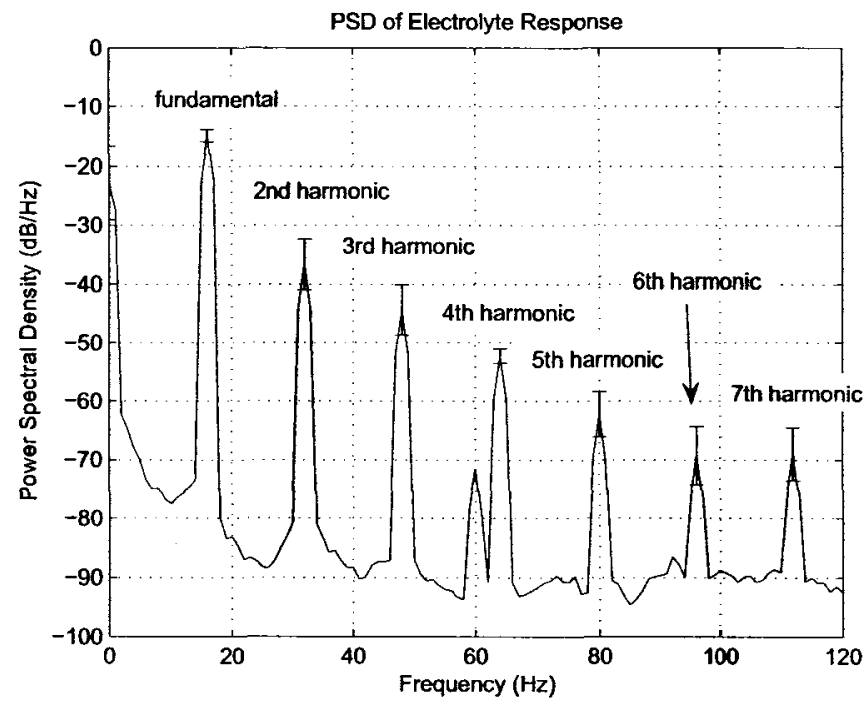

Figure 4.2: PSD of electrolyte response: The electrolyte solution showed a second harmonic greater than the third when averaged over ten seconds. The error bars, with a length of three standard deviations, provide a measure of the instability observed in the harmonics.

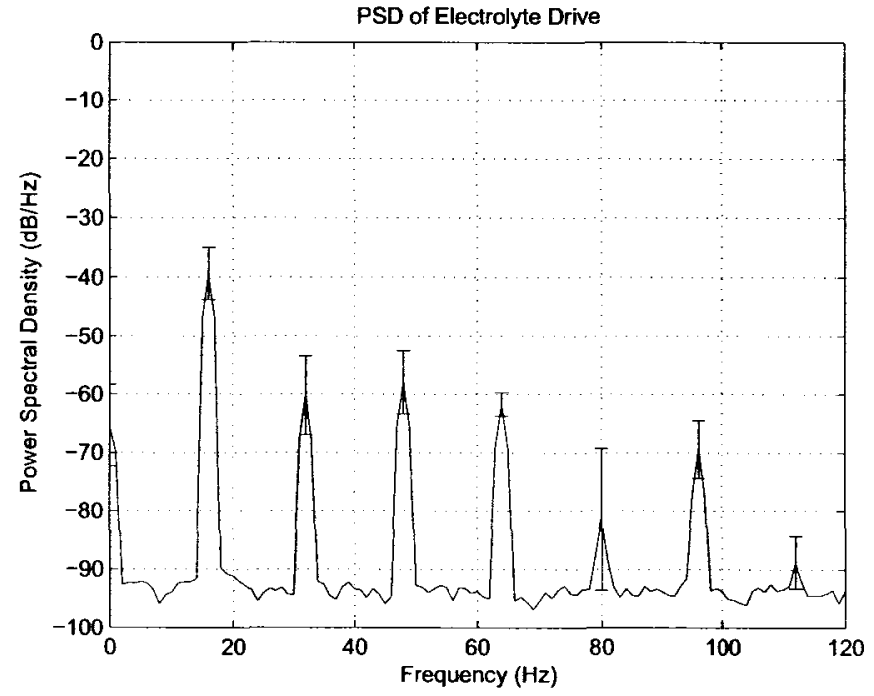

Figure 4.3: PSD of electrolyte drive: The PSD of the drive signal was calculated from the voltage signal across the resistor $R_{m}$. The error bars, with a length of three standard deviations, provide a measure of the instability observed in the harmonics. 


\section{Quiescent Yeast Suspension}

The second test case examined was that of the quiescent yeast suspension. The harmonic instability in the response signal of this case, shown in Fig 4.4, was less than that which was

observed in the electrolyte case. The PSD of the drive signal, given in Figure 4.5, displays a third harmonic magnitude greater than the second. This is quite different from the response signal, which yielded a second harmonic greater than the third. This difference between the response and drive signals indicates that spectral content can vary with impedance. The spectral component occurring at $60 \mathrm{~Hz}$ was attributed to power mains noise and was ignored in the analysis.

\section{Active Yeast Suspension}

Data collected for the active yeast suspension also showed considerable harmonic instability. The average response PSD, calculated over ten seconds two hours after the addition of glucose, is presented in Fig. 4.6. The error bars represent three standard deviations of the harmonic magnitudes, and reveal the instability described earlier. Some instability was expected, since the sample must undergo a transition from quiescent to active.

The high level of instability resulted in a fluctuation between dominant second and dominant third harmonics. The observed harmonic pattern, after averaging, did not differ greatly from the electrolyte and quiescent response cases, with harmonic magnitudes for the three test case response PSDs decreasing as harmonic number increased. Again, the power mains noise occurring at $60 \mathrm{~Hz}$ was ignored in the analysis.

The PSD of the drive signal, for which the average is shown in Fig. 4.7, also displayed instability. The second and third harmonic magnitudes are closer in value than was observed in the electrolyte and quiescent cases for the drive signals. 


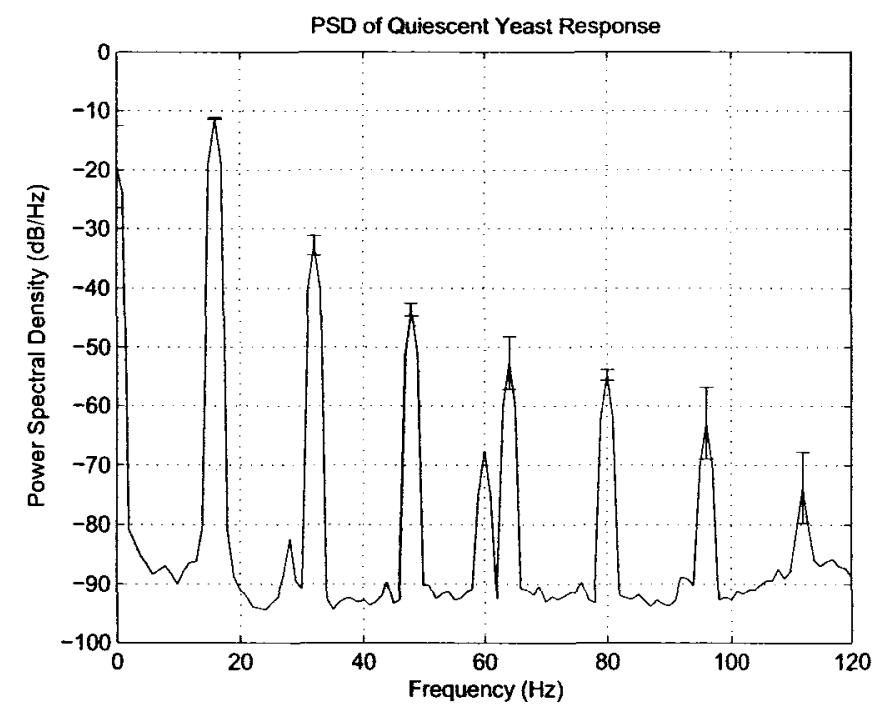

Figure 4.4: PSD of quiescent yeast response: The quiescent yeast suspension showed a second harmonic greater than the third when averaged over ten seconds. The error bars, with a length of three standard deviations, provide a measure of the instability observed in the harmonics.

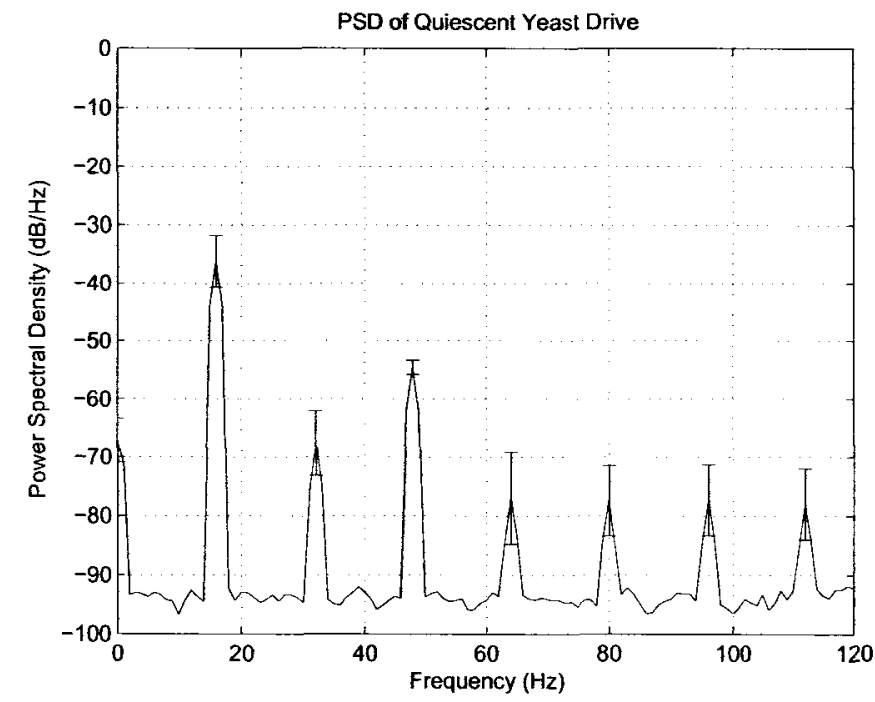

Figure 4.5: PSD of quiescent yeast drive: The quiescent yeast suspension drive signal yielded a third harmonic greater than the second when averaged over ten seconds. The error bars, with a length of three standard deviations, provide a measure of the instability observed in the harmonics. 


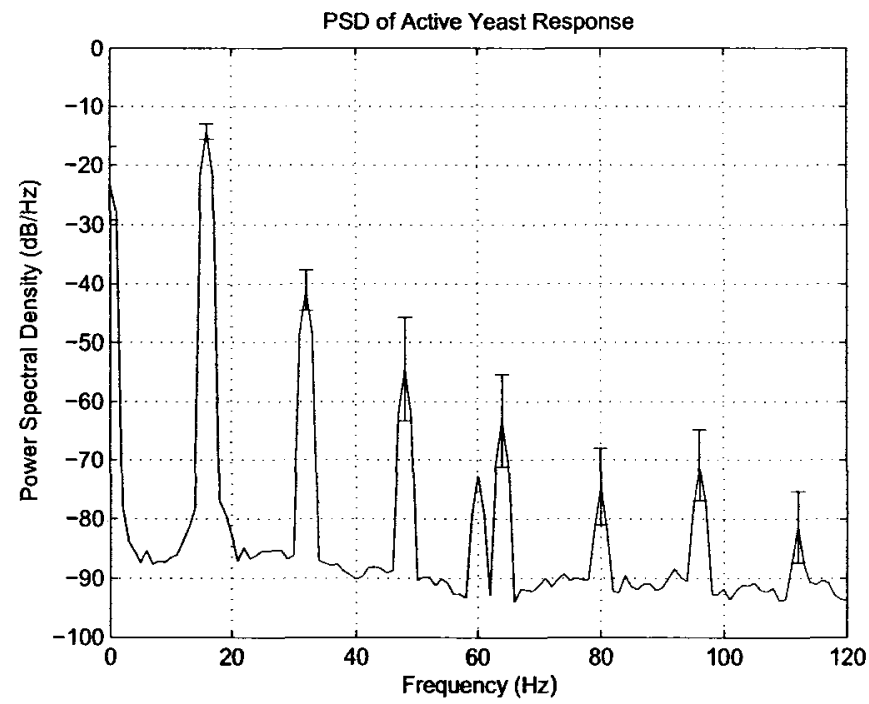

Figure 4.6: PSD of active yeast response: The PSD of the active yeast suspension response signal yielded a second harmonic greater than the third when averaged over ten seconds. The error bars, with a length of three standard deviations, provide a measure of the instability observed in the harmonics.

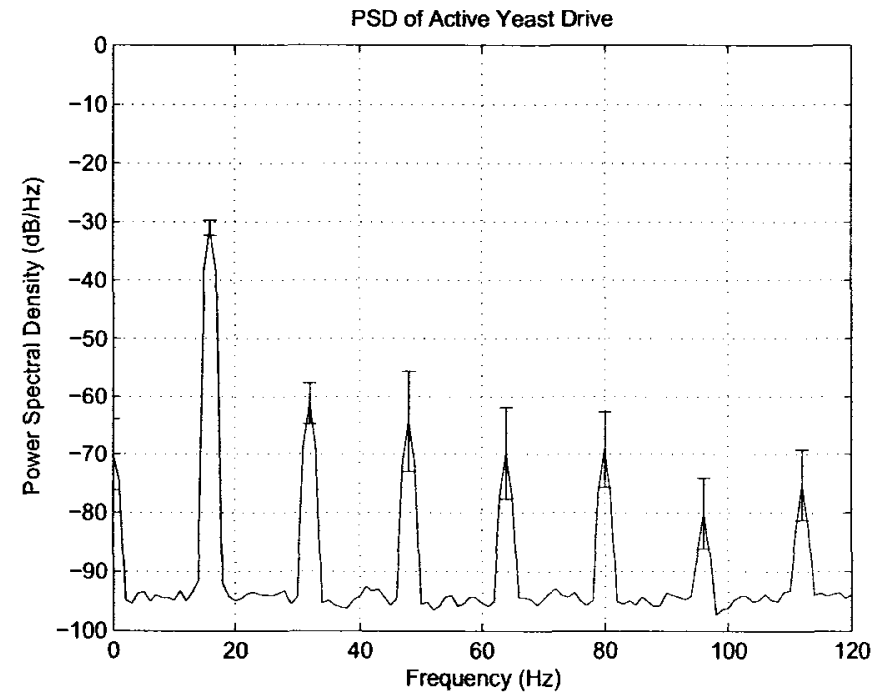

Figure 4.7: PSD of active yeast drive: The PSD of the active yeast suspension drive signal yielded a second harmonic greater than the third when averaged over ten seconds. The error bars, with a length of three standard deviations, provide a measure of the instability observed in the harmonics. 


\section{Reference Spectrum Method Results}

The reference spectrum analysis employed in [3] was repeated in this thesis work as a means to compare the experimental results with the published results. In this method (see Sec. 3.4.1) the PSD of the electrolyte response is subtracted from the respective yeast test case PSDs in the logarithmic scale.

For the quiescent case, according to [3], it was expected that the addition of yeast to the electrolyte would result in a decrease in the magnitude of the third harmonic in the response signal, while the other harmonic magnitudes would remain constant. The result of applying the reference spectrum method to the experimental quiescent data is presented in Fig. 4.8. The notable differences between the experimental results and the results of [3] are found at the third harmonic $(48 \mathrm{~Hz})$, and at the seventh harmonic $(112 \mathrm{~Hz})$. The experimental results for the third harmonic are approximately $19 \mathrm{~dB}$ higher than the published result, while the seventh harmonic is approximately $14 \mathrm{~dB}$ lower than the published result. Therefore, the quiescent case results obtained did not agree with the primary features for this case as given in [3].

For the active case, according to [3], it was expected that the application of the reference spectrum method would result in the second and fourth harmonics dominating while the other harmonic magnitudes would be negligible. The result of applying the reference spectrum method to the experimental active data is presented in Fig. 4.9. As this plot indicates the experimental results do not agree with the primary features for this case as given in [3]. 


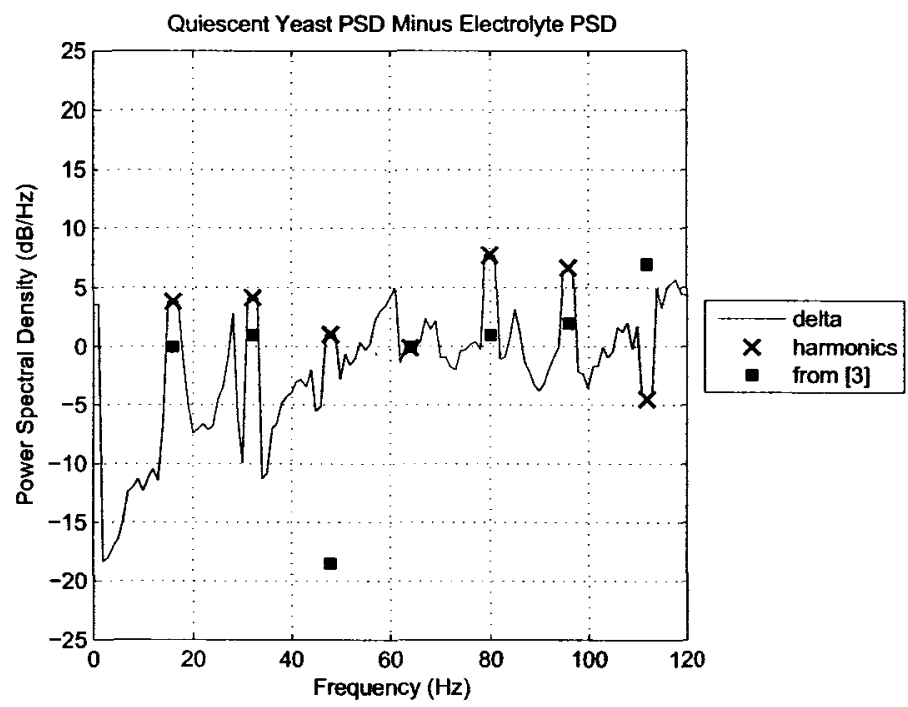

Figure 4.8: Quiescent yeast reference spectrum results: The PSD of the electrolyte response was subtracted from the quiescent yeast response PSD, and is denoted as "delta". The " $x$ " markers indicate the experimental results of the reference spectrum method at the harmonics. The $\mathbf{m a r k e r s ~ i n d i c a t e ~ t h e ~ r e s u l t s ~ o f ~ [ 3 ] . ~}$

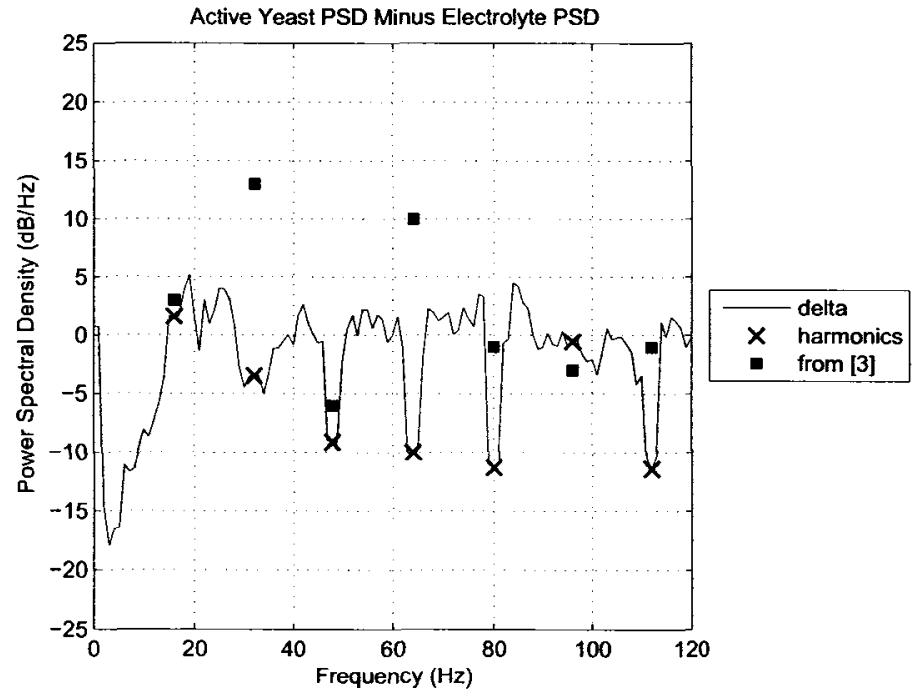

Figure 4.9: Active yeast reference spectrum results: The PSD of the electrolyte response was subtracted from the active yeast response PSD, and is denoted as "delta". The " $x$ " markers indicate the experimental results of the reference spectrum method at the harmonics. The $\mathbf{m a r k e r s}$ indicate the results of [3]. 


\subsubsection{Discussion of Results}

The results of the reference spectrum method applied to the experimental data did not agree with the results of [3]. The instability of the harmonic magnitudes in the electrolyte case makes obtaining meaningful results using this method difficult. The objective of the reference spectrum method is to remove the baseline harmonic content of the electrolyte from the yeast test cases. Since the data for each of the test cases were not recorded in parallel, it is not possible to apply the method using electrolyte and yeast data recorded at the same point in time. With the observed instability, the choice of data sets used will affect the outcome of the method. If the harmonic magnitudes of the electrolyte case were stable, then the electrolyte baseline could be subtracted without affecting the outcome.

This instability was also encountered in the experimental work of $[39,41,43]$. In [39], the authors dealt with this problem by discarding any data that showed instability, cleaning the electrodes and then restarting data collection. In the experimental work of this section, using new electrodes did not prevent the instability from occurring.

An additional problem with recording data sequentially rather than in parallel is that ambient conditions and noise can differ between the test cases. Differences in temperature can alter the conductivity of the samples [35]. The reference spectrum method as applied in [3] required that conductivity for the samples be matched.

To counter the observed instability, the first improvement that must be made to the experiment is to enable the collection of data for the test cases in parallel. The data acquisition system used in this initial work has the shortcoming of being unable to reliably record multichannel data. This prohibits the comparison of the different test case signals at the same point in time. Additionally, this shortcoming prohibited recording data for extended periods, as the drive and response channels had to be sampled sequentially. Recording 
data over a longer time period will allow any transient behavior to stabilize, reducing the instability of the harmonic magnitudes.

The following Sec. 4.5 continues with the NLDS work, with a modified apparatus that enabled data collection in parallel over an extended time period.

\subsection{Parallel Experimental Work}

\subsubsection{Improvements}

In light of the system instability problem found in the initial set of experiments, a second set of experiments was run using an improved protocol with more flexible data acquisition hardware. This allowed data to be collected more frequently and over a much longer time period without any system disturbance.

The second experimental setup used a DAQPad 6020E data acquisition device (National Instruments DAQPad 6020E, National Instruments Corp., Austin, Texas) connected to a PC for data acquisition. This equipment was capable of sampling multiple channels consecutively. Amplification, sampling, and analog to digital conversion were done with this new device using twelve bit resolution, operating at a sampling rate of $2048 \mathrm{~Hz}$. The DAQPad was controlled through the MATLAB software environment (MATLAB 7.0.4, The Mathworks Inc., Natick, Massachusetts), which enabled the interleaving of data collection and analysis. This simplified the automation of data collection and processing, allowing data to be collected overnight.

To more accurately reveal the variation in the PSD estimate, the length of data record was reduced from two minutes to two seconds. This record length allowed 38 cycles at the fundamental frequency to be included in the PSD estimate. Since the seventh harmonic is the highest harmonic of interest in NLDS of yeast samples, at a fundamental frequency of 
$16 \mathrm{~Hz}$, the highest frequency of interest is $133 \mathrm{~Hz}$. Thus the sampling rate of $2048 \mathrm{~Hz}$ is well above the Nyquist frequency. Data records were taken every seven seconds. The total duration of each experiment was sixteen hours.

The sequential experimental work of Sec. 4.4.2 used only one sample container. This limitation meant that different test cases could not run in parallel; rather, data were collected on different days for the different test cases. A significant improvement to the experimental system was the assembly of four separate sample containers, each with a separate four electrode configuration. Four independent drive current monitoring resistors were also included, with resistance matched to better than $1 \%$. The four new drive circuits were connected in parallel to the stimulating voltage source, ensuring each sample would experience the same $\mathrm{AC}$ signal. Two data acquisition channels were used per sample container; one for the response electrodes, and one for the current monitoring resistor. In total, eight channels were required. The data acquisition and processing software were modified so that four separate test cases could be run in parallel; all with a common voltage source but with independent data acquisition channels. Running the test cases in parallel had the primary advantage of allowing tests to be performed under the same environmental conditions, which makes comparisons between them more accurate. Additionally, this reduced the number of days required to collect data by a factor of four.

\section{Presentation of Data}

To analyze the 8000 records per channel produced over a sixteen hour period, algorithms were developed in the MATLAB environment to perform this task. Data were recorded in the time domain and saved to disk. The PSD was then estimated as described in Section 4.2.3. The maximum value of the spectrum, within a narrow window centered at the fre- 
quencies of interest (harmonics two through seven, as well as at the fundamental and at DC), were then determined. These harmonic magnitudes were then plotted over time.

This analysis method simplifies the analysis of the three dimensional time-frequency data illustrated in the example shown in Fig. 4.10. This three dimensional plot shows frequency on the $x$-axis, time (normalized to one hour) on the $y$-axis, and the magnitude of the PSD on the z-axis. The peaks at the fixed and known harmonic frequencies contain the data needed. These peaks at the harmonic frequencies have magnitudes which vary with time. Since the harmonic frequencies are known, the analysis method can omit the frequency axis with no loss of information. Only the peak values of the harmonics over time need

to be shown. This effectively transforms the time-frequency data illustrated in Fig. 4.10 to a time-course representation of harmonic magnitudes, to simplify data analysis. These time-course plots are used in the remainder of this thesis to analyze harmonic magnitude variation over time.

The results for the test cases described earlier in Section 4.3 are discussed in the following section, as well, additional control experimental are presented. The improved experimental protocol is able to clearly show the behavior of the test cases over an extended period of time. Since the second and third harmonics are the harmonics of main interest, the plots presented in this section are simplified to include only the fundamental, second, and third harmonics.

\subsubsection{Parallel Experimental Results}

Three trials were run while data were recorded for both the drive and response channels. Each trial sampled data from the electrolyte test case, the quiescent yeast test case, and the active yeast test case in parallel. The plots in which this data are presented are grouped 


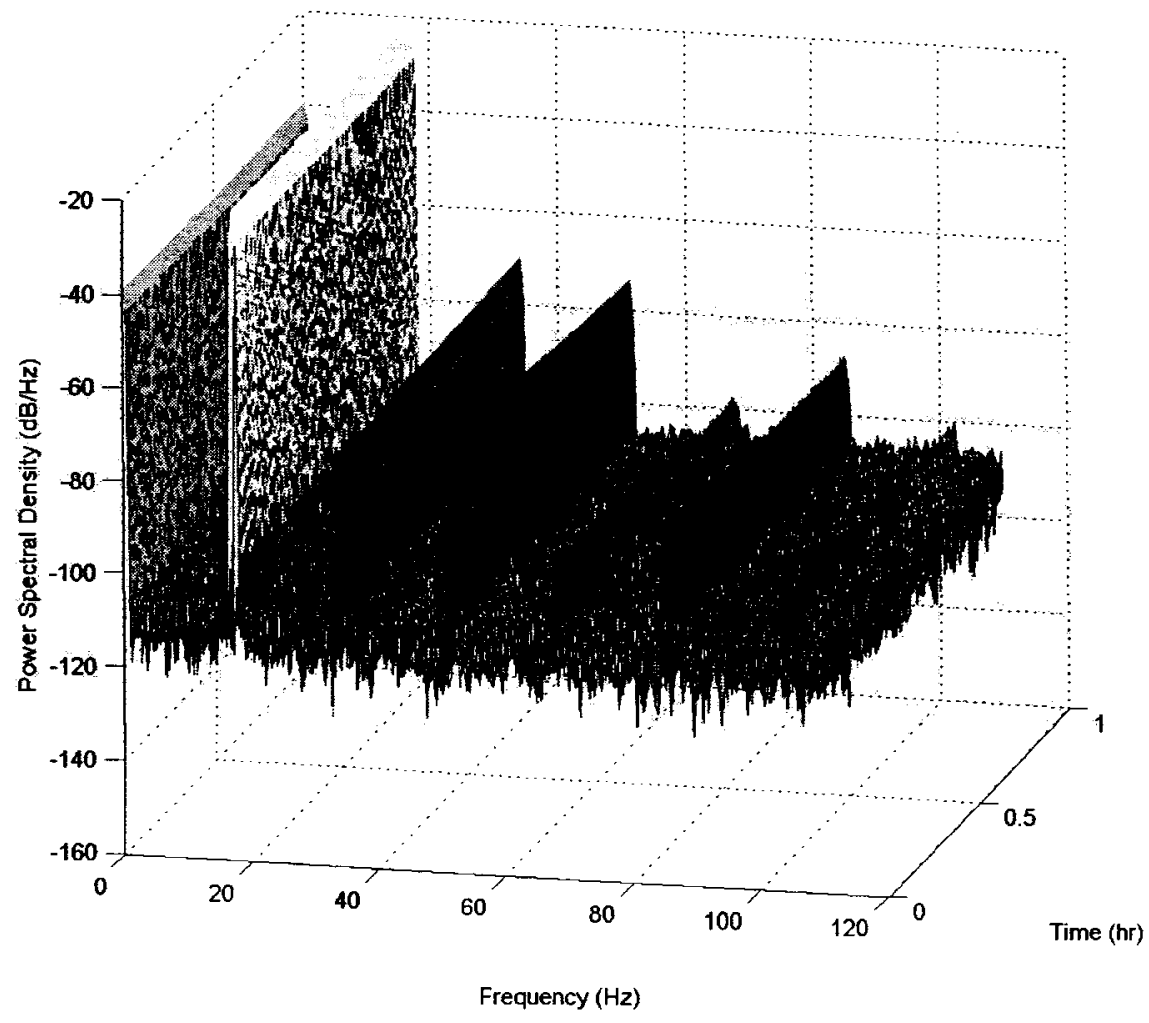

Figure 4.10: Three dimensional time-frequency representation. 
according to test case, rather than by trial. To reveal the relationship between the test cases of each trial, the results of applying the reference spectrum method of [3] will later be presented.

\section{Electrolyte Medium}

The electrolyte test case data for the response channel are first presented in Fig. 4.11. Some harmonic instability is apparent in the first fifteen minutes of this test case. After this initial instability the magnitudes do not remain constant, but instead tend to drift. This drift behavior is not the same when the test cases are compared. The magnitudes reveal a clear trend in terms of relative magnitude, with the fundamental dominating, followed by the second and third harmonics respectively.

The electrolyte drive signal harmonics, given in the plots of Fig. 4.12, display the initial instability and drift behavior as is observed in the response data. Each test case is unique in terms of drift and relative difference between the second and third harmonics.

\section{Quiescent Yeast}

The quiescent yeast test case data for the response channel are presented in Fig. 4.13. The harmonic magnitudes remain relatively constant, with only trial 3 showing initial instability. The pattern of relative harmonic magnitude is similar to that of the electrolyte case with a dominant fundamental, followed by the second and third harmonics respectively.

The quiescent yeast drive signal harmonics, given in the plots of Fig. 4.14, display a different harmonic pattern than is observed in the response data. In these data the fundamental is dominant, followed by the third and second harmonics respectively. 

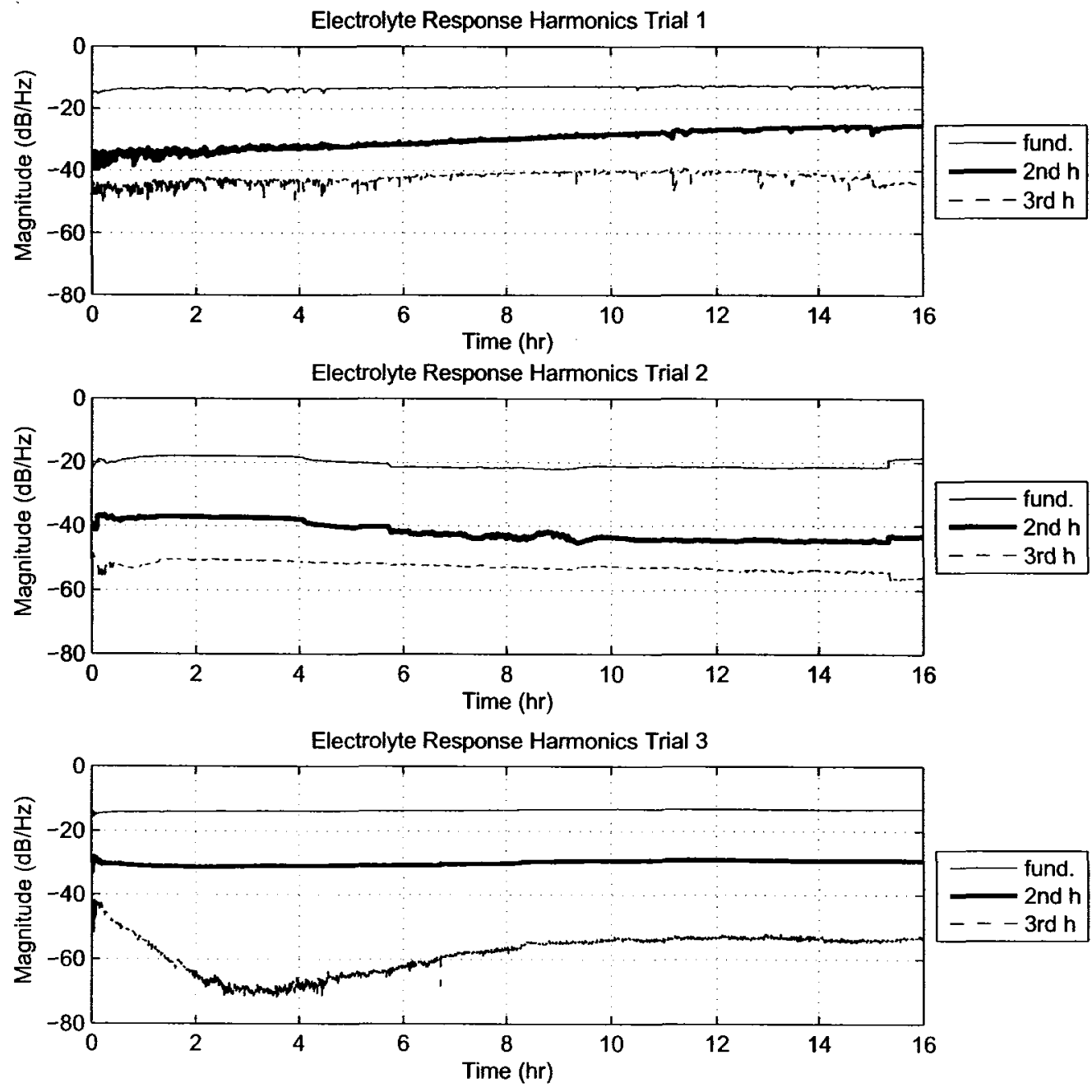

Figure 4.11: Electrolyte response time-course spectral results: The time course magnitudes of the fundamental, second harmonic, and third harmonic are shown for three electrolyte test cases as observed at the recording electrodes. Trial 1 is shown in the top plot, while the middle and bottom plots are for the second and third trials respectively. All plots show a similar trend in terms of the relative magnitudes of the second and third harmonics. 


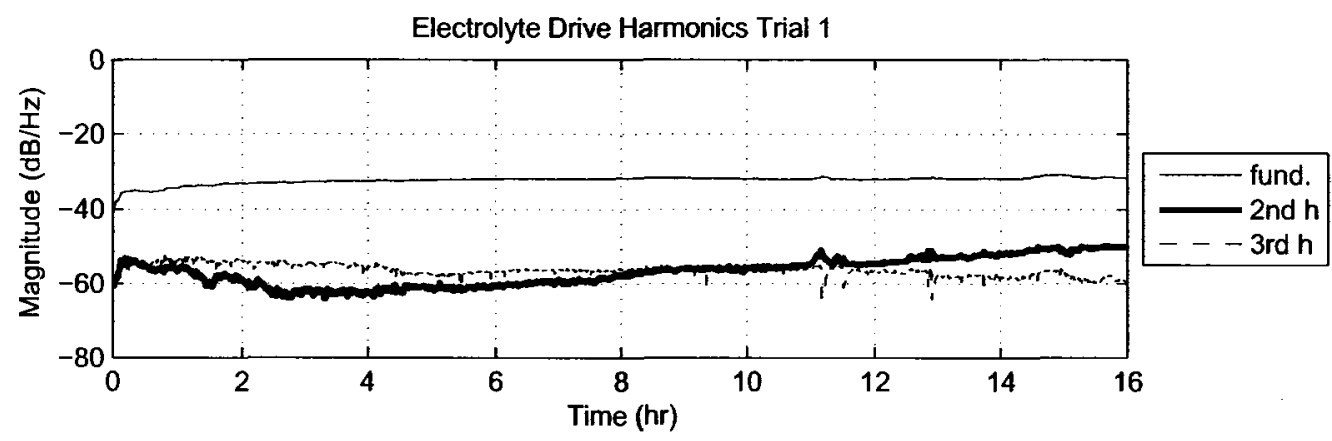

Electrolyte Drive Harmonics Trial 2

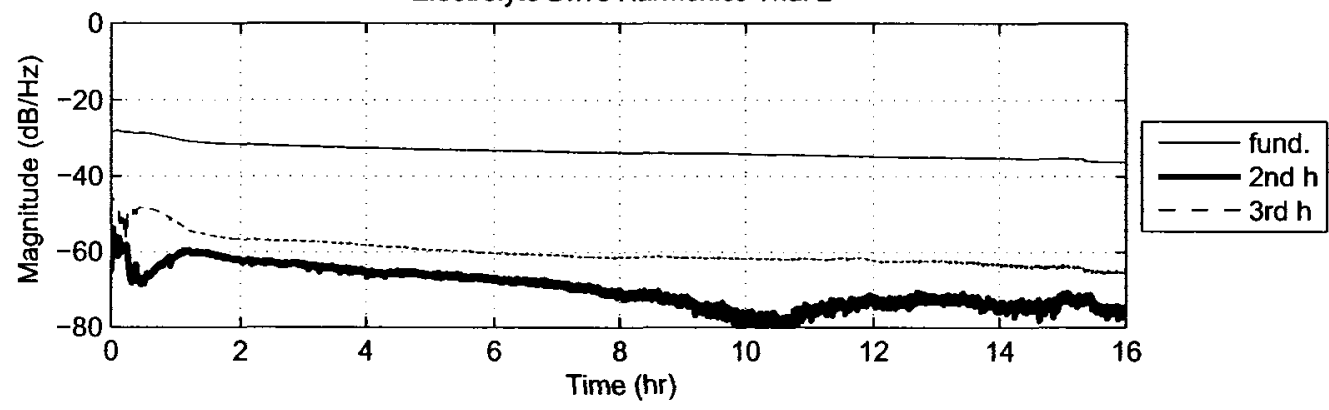

Electrolyte Drive Harmonics Trial 3

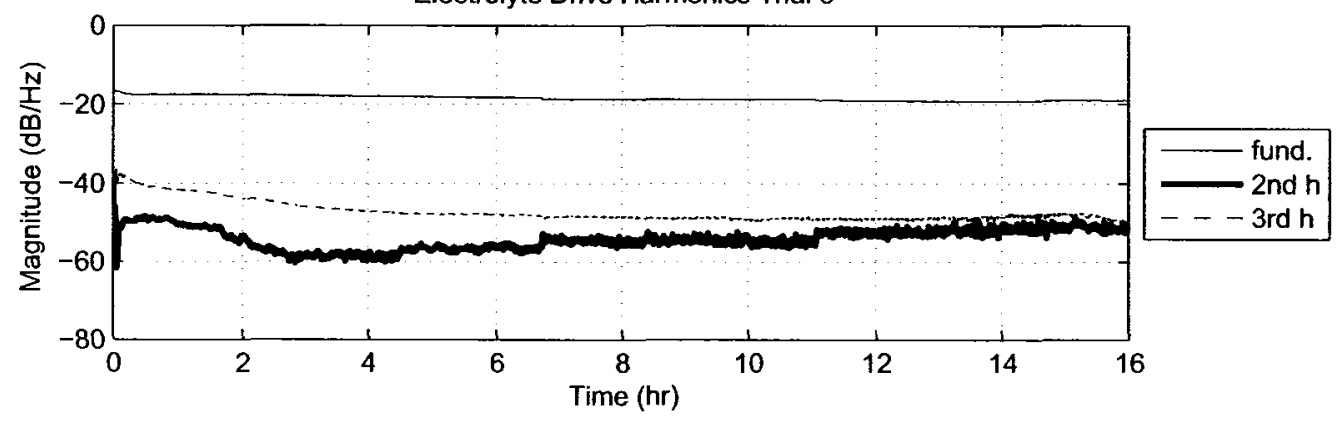

Figure 4.12: Electrolyte drive time-course spectral results: The time course magnitudes of the fundamental, second harmonic, and third harmonic are shown for three electrolyte test cases as observed at the current monitoring resistor. Trial 1 is shown in the top plot, while the middle and bottom plots are for the second and third trials respectively. 

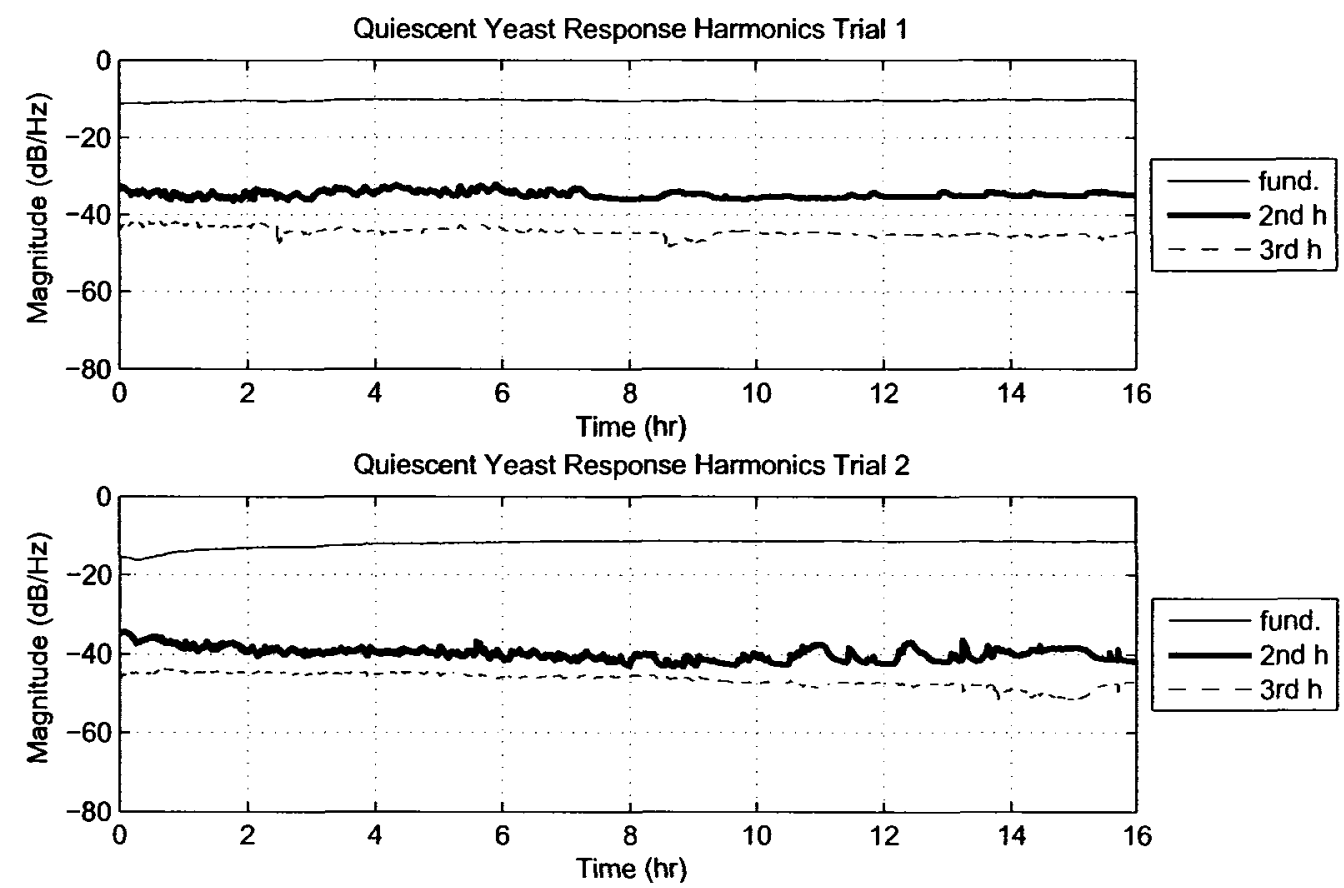

Quiescent Yeast Response Harmonics Trial 3

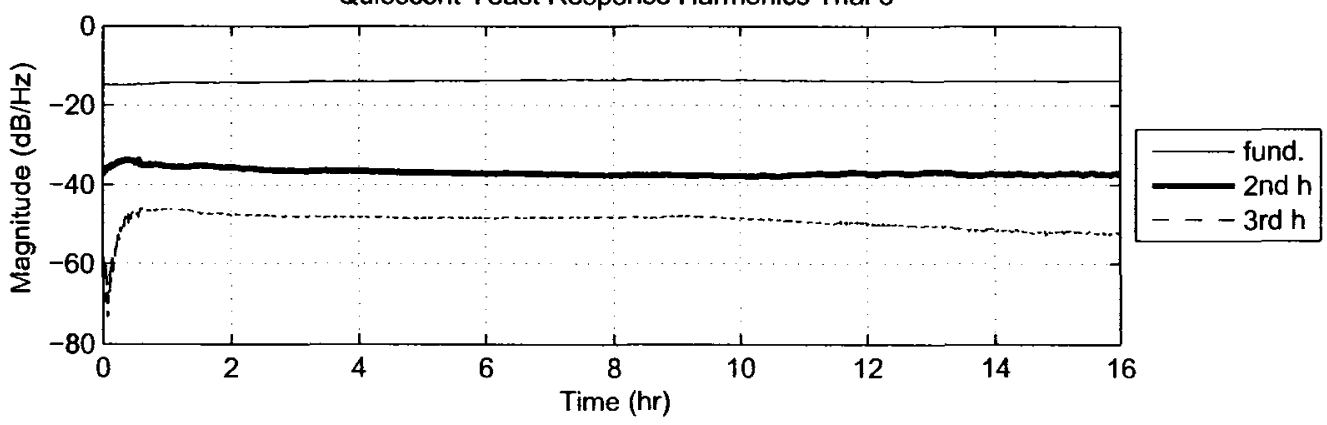

Figure 4.13: Quiescent yeast response time-course spectral results: The time course magnitudes of the fundamental, second harmonic, and third harmonic are shown for three quiescent yeast test cases as observed at the recording electrodes. Trial 1 is shown in the top plot, while the middle and bottom plots are for the second and third trials respectively. All plots show a similar trend in terms of the relative magnitudes of the second and third harmonics. 

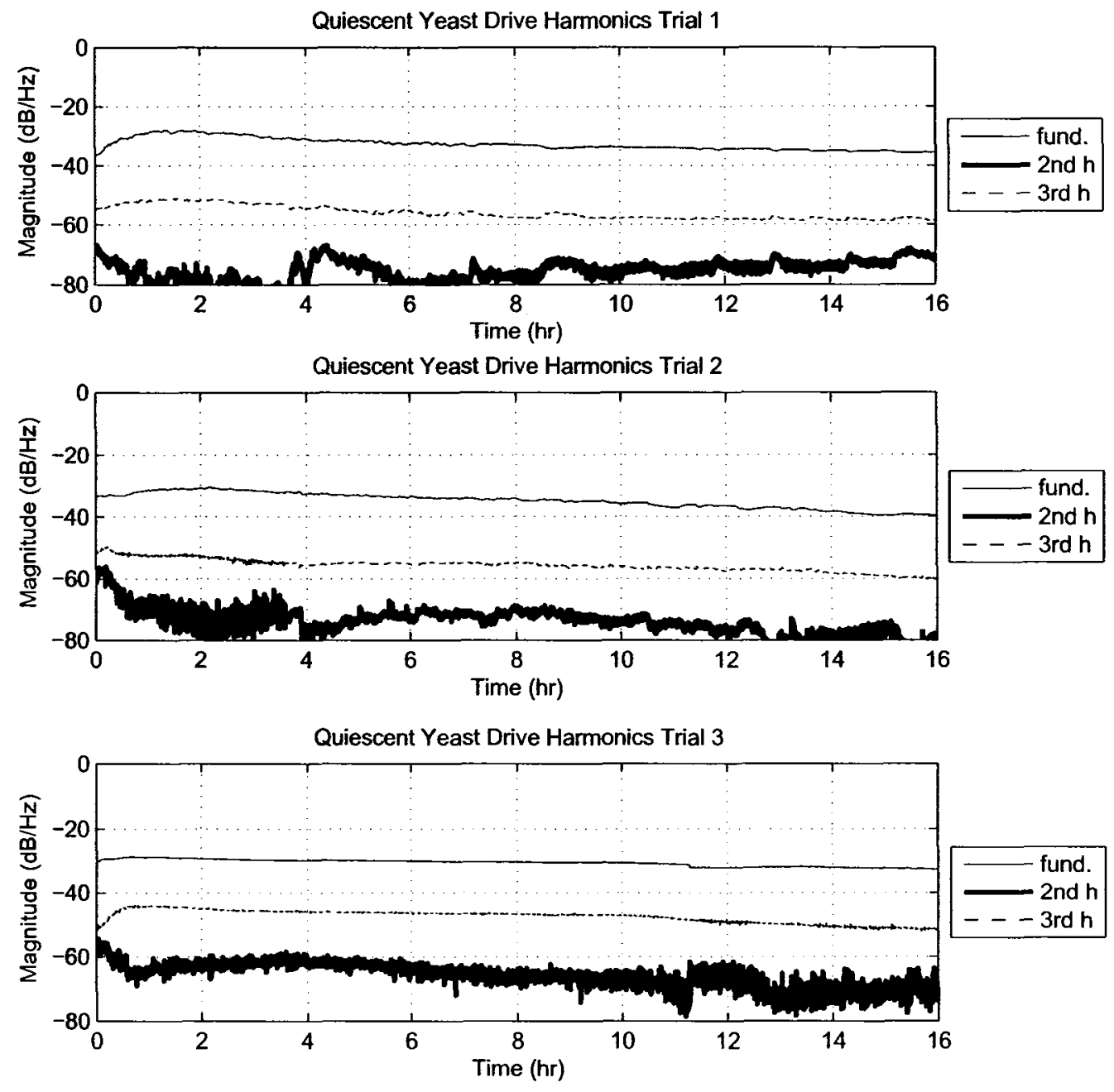

Figure 4.14: Quiescent yeast drive time-course spectral results: The time course magnitudes of the fundamental, second harmonic, and third harmonic are shown for three quiescent yeast test cases as observed at the current monitoring resistor. Trial 1 is shown in the top plot, while the middle and bottom plots are for the second and third trials respectively. All plots show a similar trend in terms of the relative magnitudes of the second and third harmonics. 
Active Yeast

The active yeast test case data for the response channel are presented in Fig. 4.15. In this test case, initial instability is observed until approximately hour 2 in trials 1 and 3, and until approximately hour 3.5 in trial 2 . After the initial instability, the harmonic magnitudes show a small variation, with the fundamental increasing by at least $3 \mathrm{~dB}$. A clear harmonic pattern in terms of relative magnitude is apparent in the response signal of this test case, with the fundamental dominating, followed by the second and third harmonics respectively.

The active yeast drive signal harmonics, given in the plots of Fig. 4.16, display a different harmonic pattern than is observed in the response data. In the first and second trials the fundamental is dominant, followed by the third and second harmonics respectively, after a period of initial instability. In the third trial the latter half of the test displays a second and third harmonic of approximately the same magnitude. The first half of the test shows two reversals in terms of dominant second and third relative harmonic magnitudes.

\section{Reference Spectrum Method Results}

To provide a means of comparing the data to that of [3], the reference spectrum method described in Sec. 3.4.1 and applied in the previous experimental work (see Sec.4.4.2) was applied to these data. In the reference spectrum method, the PSD of the electrolyte response is subtracted from the active yeast PSD in logarithmic scale. The results of applying the reference spectrum method to the parallel experimental data for the quiescent case are presented in Fig. 4.17, and for the active yeast in Fig. 4.18. In these figures, the top plot shows the results of [3], extrapolated over the 16 hour test period based on the 10 seconds of data presented in the paper.

According to [3], it was expected that the quiescent yeast, as is shown in the top plot, 


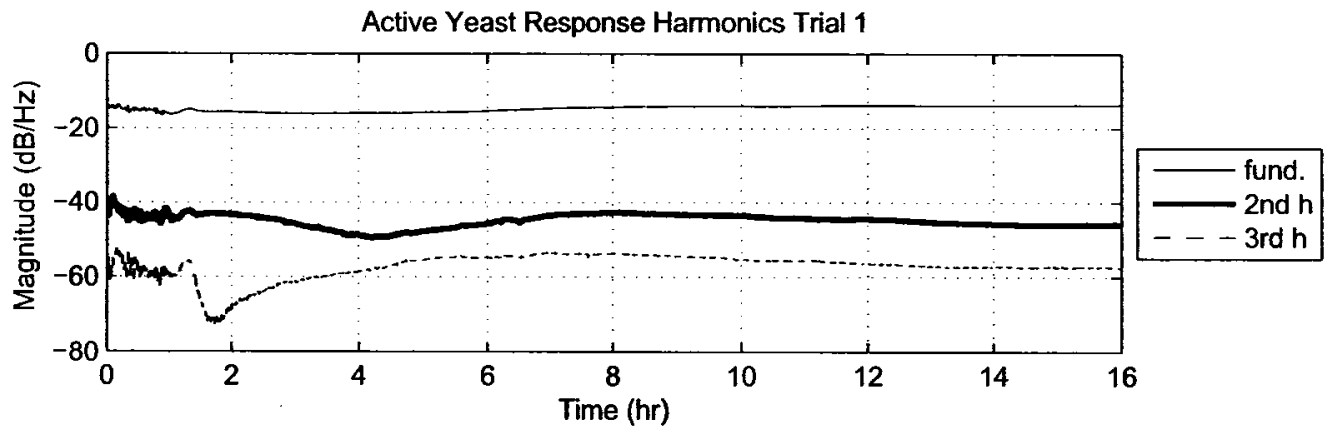

Active Yeast Response Harmonics Trial 2

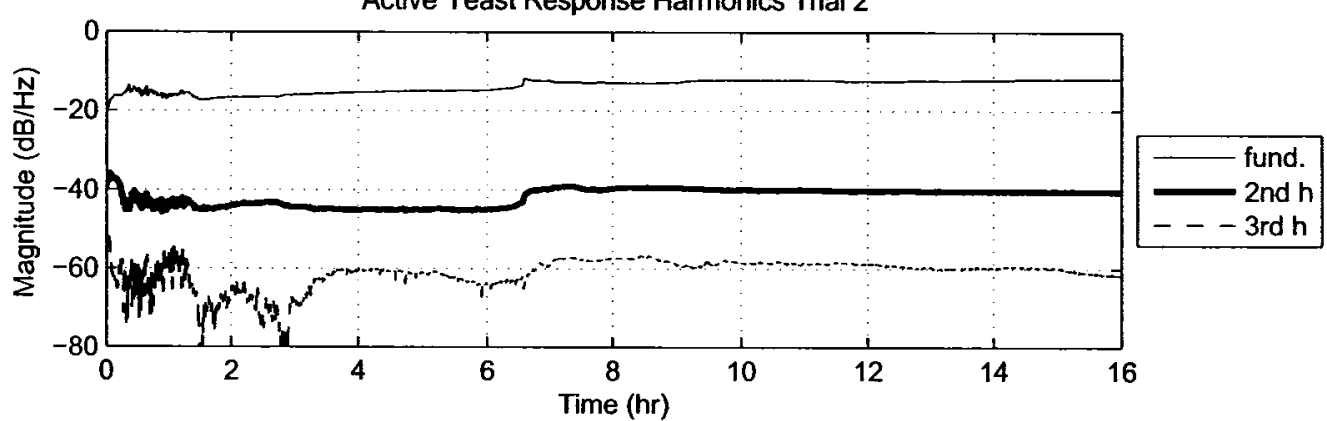

Active Yeast Response Harmonics Trial 3

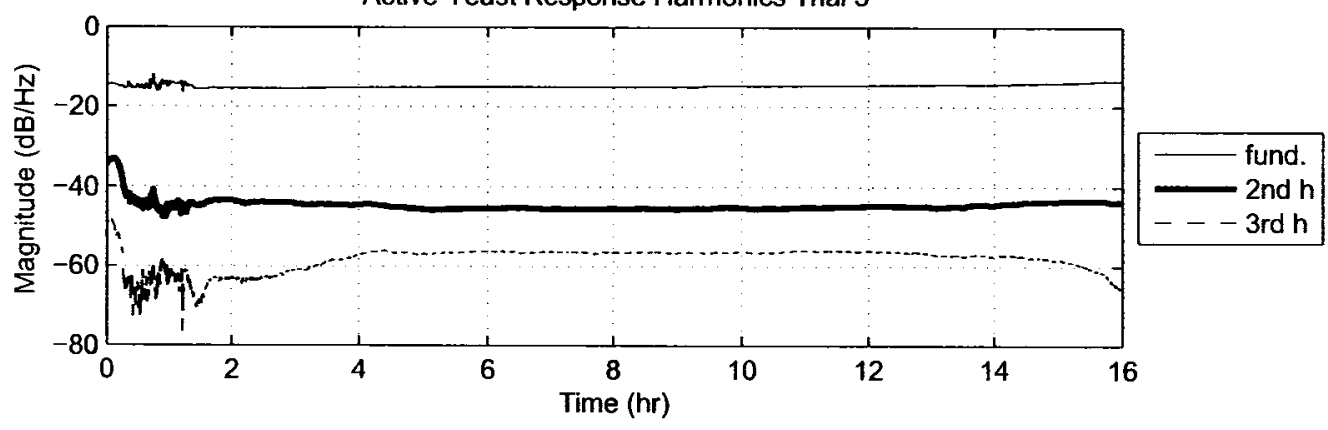

Figure 4.15: Active yeast response time-course spectral results: The time course magnitudes of the fundamental, second harmonic, and third harmonic are shown for three active yeast test cases as observed at the recording electrodes. Trial 1 is shown in the top plot, while the middle and bottom plots are for the second and third trials respectively. All plots show a similar trend in terms of the relative magnitudes of the second and third harmonics. 


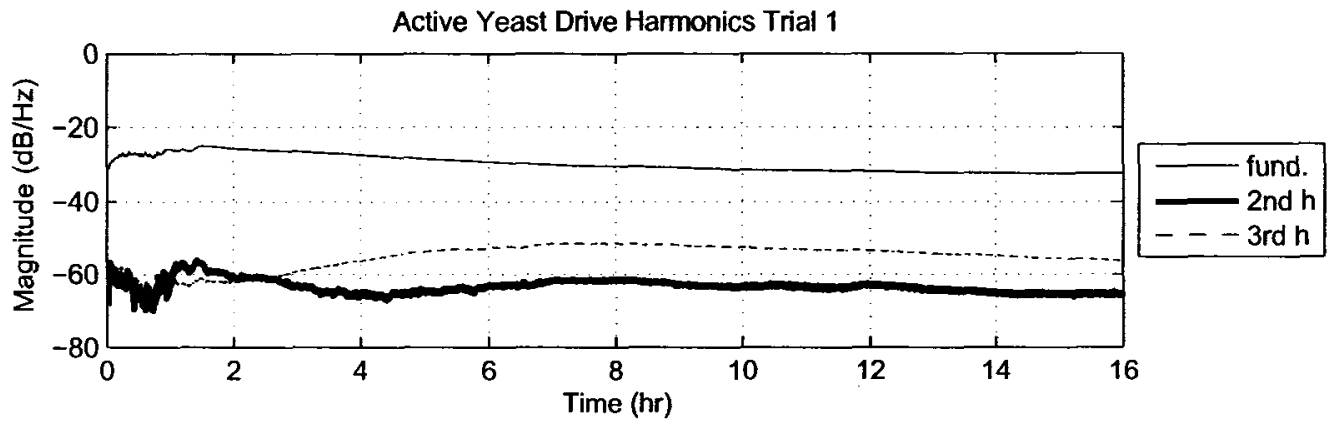

Active Yeast Drive Harmonics Trial 2

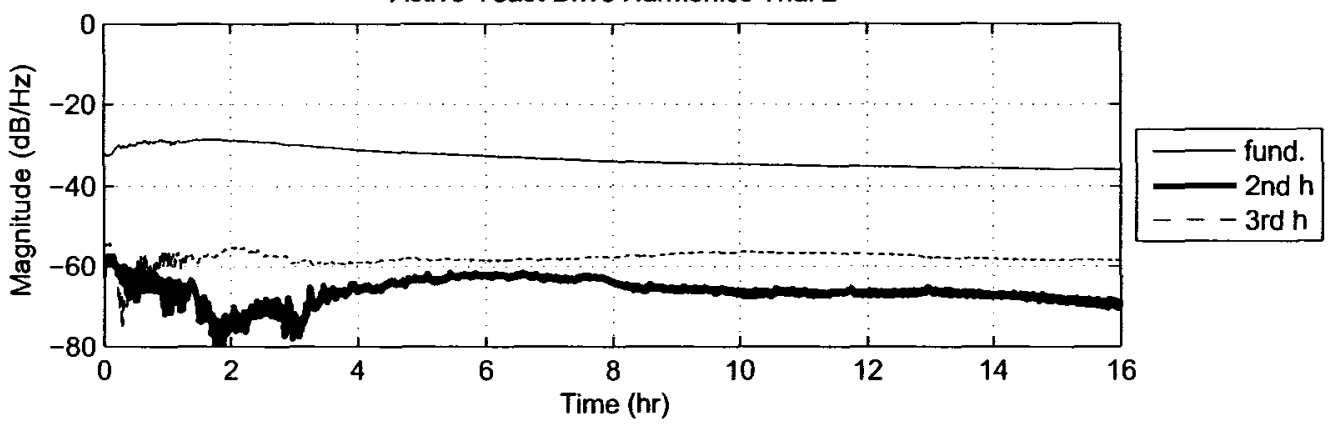

Active Yeast Drive Harmonics Trial 3

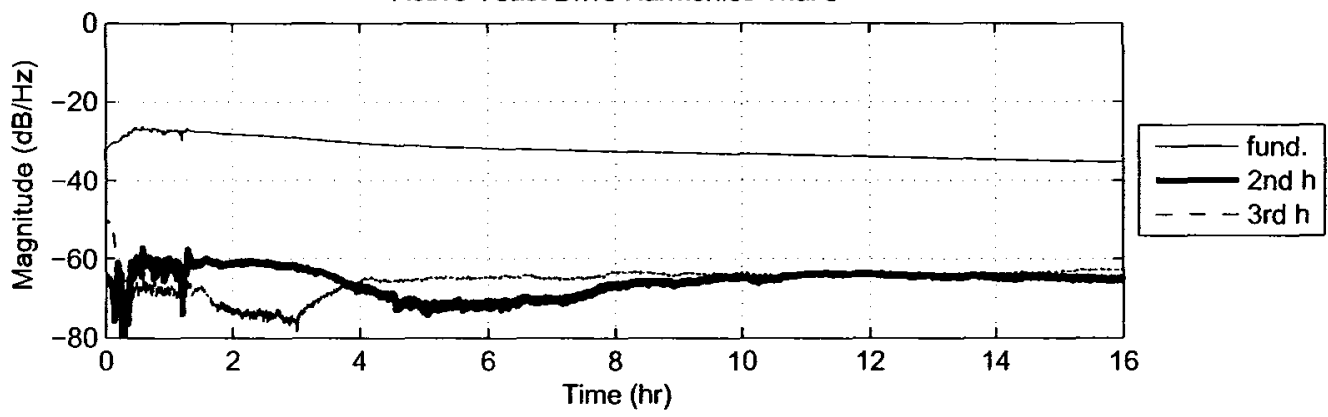

Figure 4.16: Active yeast drive time-course spectral results: The time course magnitudes of the fundamental, second harmonic, and third harmonic are shown for three active yeast test cases as observed at the current monitoring resistor. Trial 1 is shown in the top plot, while the middle and bottom plots are for the second and third trials respectively. 
would exhibit a strong negative third harmonic in the reference spectrum results. This was not observed in any of the three trials. For the active yeast case, the results of [3] are characterized by a strong positive second harmonic and a weaker negative third harmonic (see the top plot of Fig. 4.9). The active yeast results of this experimental work did not display this characteristic.

\section{Effect of Boiling Yeast}

To examine the time-course spectral components of a suspension of dead cells, samples were prepared by boiling a suspension of quiescent and active yeast. Temperatures of 50 ${ }^{\circ} \mathrm{C}$ are required to kill yeast cells, and will also result in the thermal denaturation (loss of structural shape) of cell proteins [44]. The tough cell wall of the yeast S. cerevisiae typically requires more aggressive measures than boiling to lyse [44]. Therefore, the effect of boiling the yeast cells will result in the cell macro-structure being maintained, while the proteins such as the $\mathrm{H}^{+}$ATPase will lose their structural shape and cease to function.

Samples were prepared by gently boiling the test suspension for three minutes at 100 ${ }^{\circ} \mathrm{C}$, allowing the suspension to cool, and then adding distilled water to replace that lost through evaporation. The purpose of this test is to determine if a suspension of denatured dead cells exhibits the same properties as a live suspension. The time-course response PSD data for the boiled suspensions are shown in the top plot of Fig. 4.19.

The boiled quiescent yeast exhibits less noise in the third harmonics as compared to the living quiescent suspensions shown earlier in Fig. 4.13. The harmonic pattern in terms of relative magnitude is similar between the living and boiled, with a the fundamental dominating, followed by the second and third harmonics respectively. This indicates that the boiled quiescent yeast is slightly more stable at the third harmonic than a living quiescent 

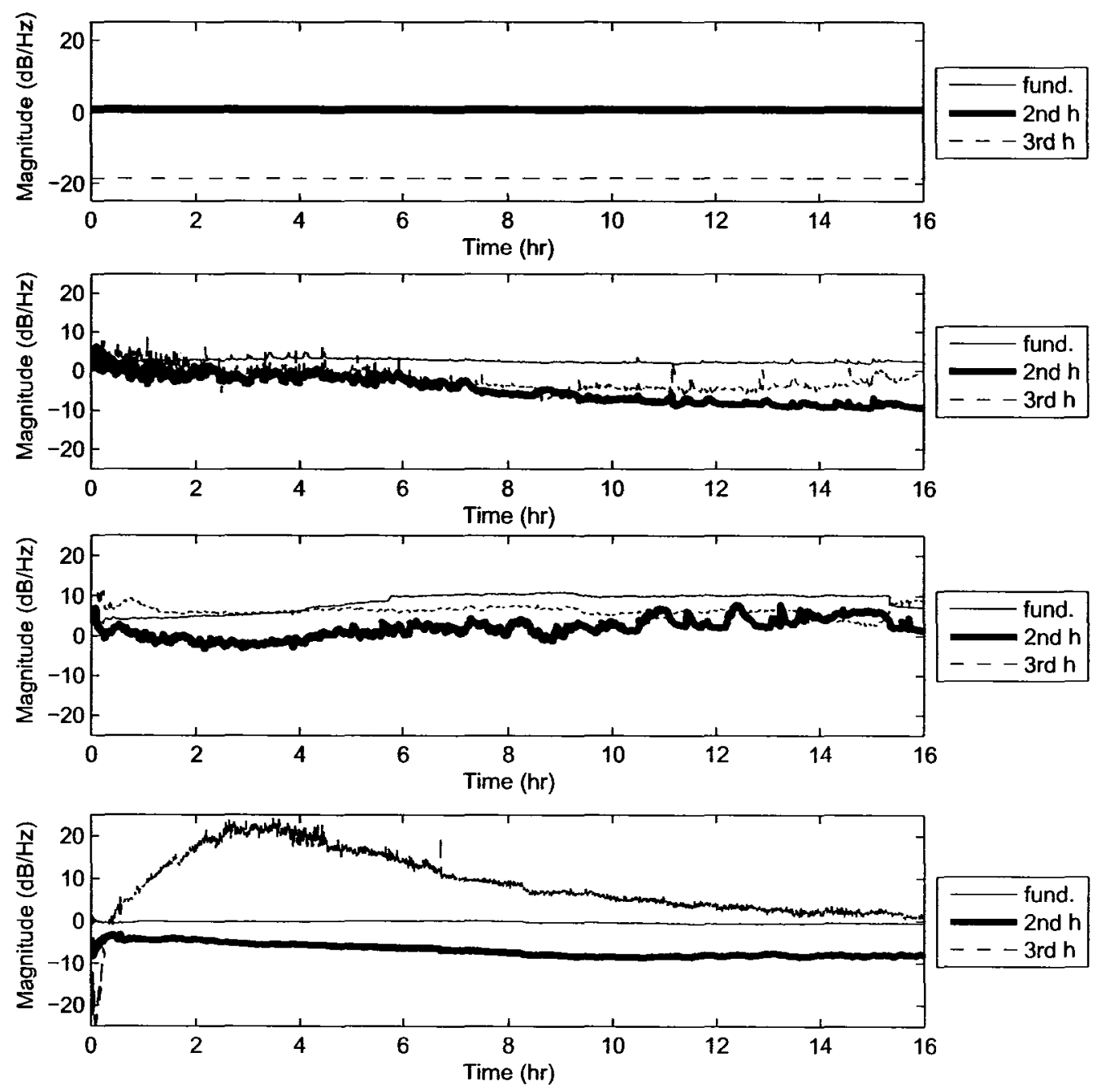

Figure 4.17: Quiescent yeast reference spectrum time-course results: The time course magnitudes of the reference spectrum output for the three quiescent yeast trials are shown in the lower three plots. The top plot shows the results of [3], extrapolated over the 16 hour test period based on the 10 seconds of data presented in the paper. In this top plot the fundamental and the second harmonic are at approximately the same magnitude. The lower three plots, in descending order, are for trials one through three. From these plots it is apparent that the experimental results for the quiescent yeast do not agree with those of [3]. 

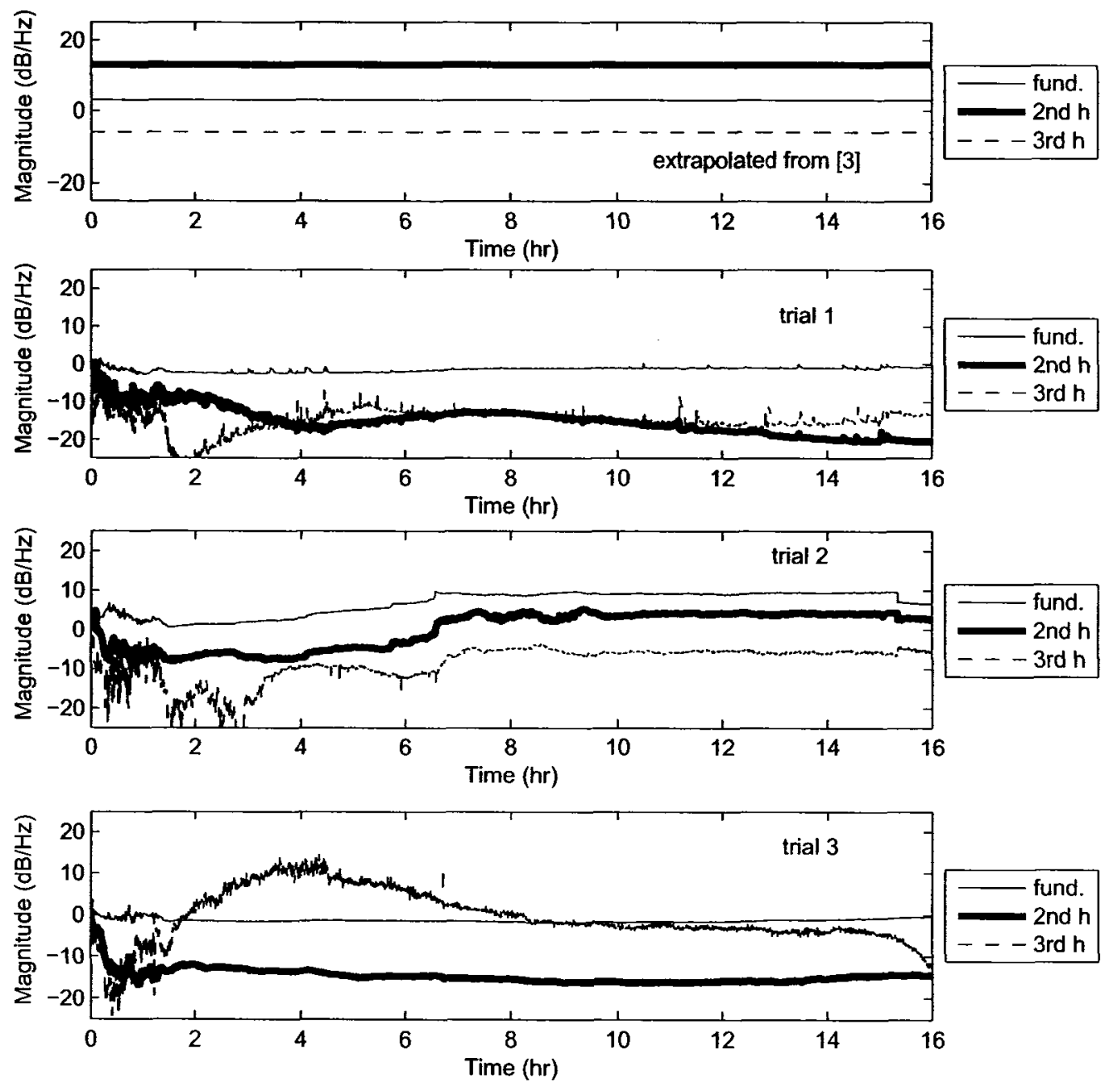

Figure 4.18: Active yeast reference spectrum time-course results: The time course magnitudes of the reference spectrum output for the three active yeast trials are shown in the lower three plots. The top plot shows the results of [3], extrapolated over the 16 hour test period based on the 10 seconds of data presented in the paper. The lower three plots, in descending order, are for trials one through three. From these plots it is apparent that the experimental results for the active yeast do not agree with those of [3]. 
suspension, but that the two do not display a notably different nonlinear behavior.

The boiled active yeast sample does not exhibit the considerable harmonic variation observed in the living active yeast suspension. This comparison can be made by visually examining the boiled yeast data presented in the bottom plot of Fig. 4.19 and the data for the active yeast trial shown earlier in Fig. 4.15. This examination indicates that a different nonlinear behavior occurs in a living active suspension than occurs in a dead active suspension.
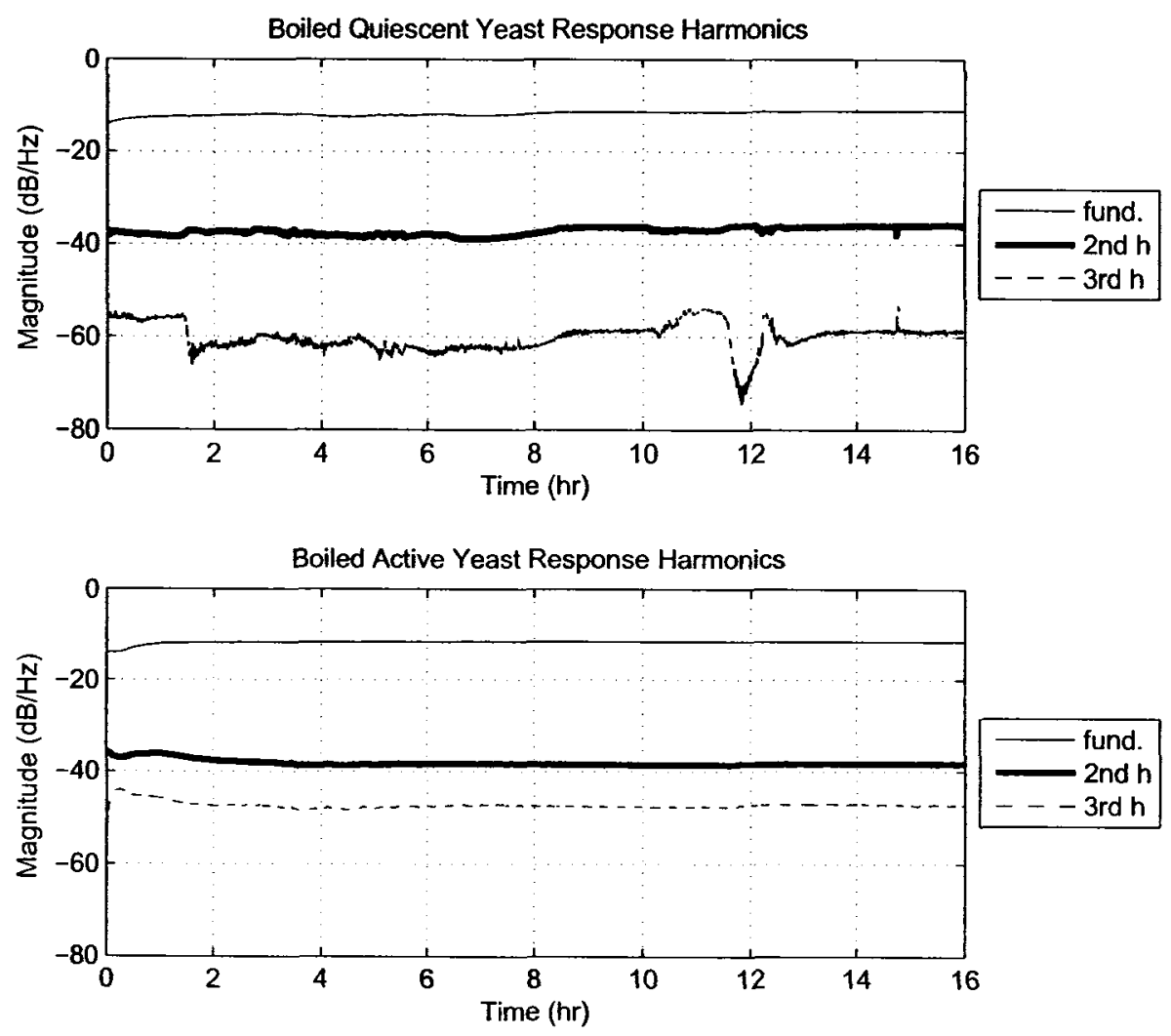

Figure 4.19: Effect of boiling yeast: The top plot shows the response of a boiled suspension of formerly quiescent yeast. The bottom plot shows the response of a boiled suspension of formerly active yeast. 


\section{Effect of Glucose on Electrolyte}

The next control experiment was performed to examine the effect of adding glucose to the electrolyte medium and comparing it to the electrolyte without glucose. The purpose of this experiment was to determine if glucose itself was responsible for the variation in harmonic magnitudes, rather than a biological response to glucose. As the the plots of Fig. 4.20 indicate, the harmonic magnitudes are similar for the electrolyte whether glucose is present or not. The electrolyte with glucose added does show some initial instability in the first hour, after which it closely matches the harmonic pattern for the no glucose test case. From this it can be concluded that glucose alone does not change the nonlinear nature of the system.

\subsubsection{Discussion of Results}

This set of experiments in which data for the different test cases were recorded in parallel over sixteen hours provides a superior means of examining the relative harmonic magnitudes of the test cases as compared to the protocol used in [3] and in Sec. 4.4.2. Acquiring data every six seconds over an extended time period enables observing phenomena such as instability and drift in harmonic magnitudes, and provides a better indication of system behavior as compared to averaging the PSD over ten seconds at one time point.

The first aspect to consider is the initial instability observed in some of the trials. In the work of this section, the test samples were not disturbed nor were the electrodes changed after the experiment began. In the previous work of Sec. 4.4.2, new electrodes were used every thirty minutes and the samples were stirred. A large standard deviation in harmonic magnitude was typically observed in the samples of Sec. 4.4 .2 which had been disturbed. In the work of this section, where initial instability was observed in cases other than the active 

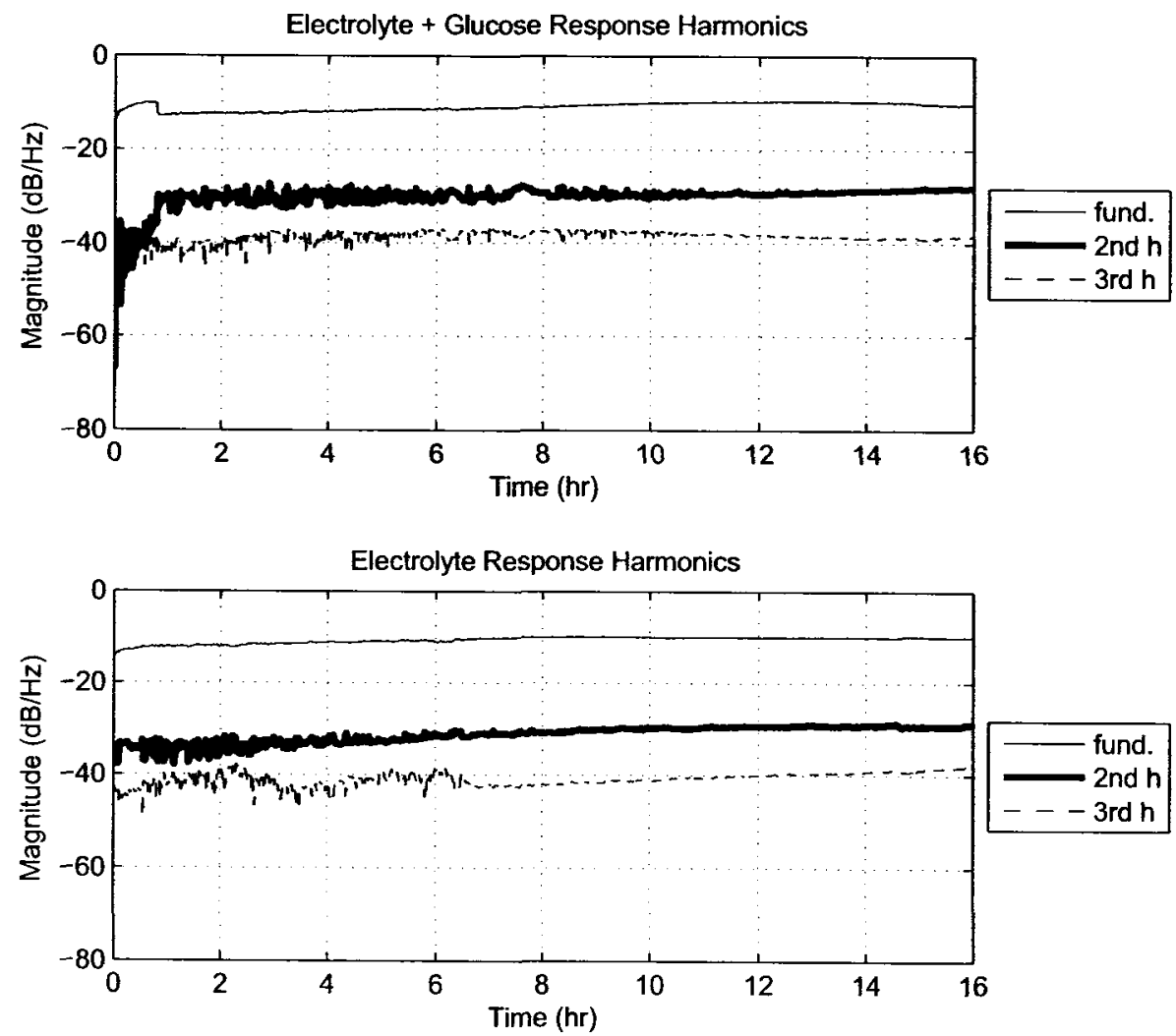

Figure 4.20: Effect of glucose on electrolyte: The top plot shows the response of the electrolyte medium. The bottom plot shows the response of the electrolyte medium with a glucose concentration of $170 \mathrm{mmol} \cdot \mathrm{L}^{-1}$ added. Data were recorded in parallel. 
yeast, it tended to stabilize in the first hour. This suggests that either the electrodes require a stabilization period, or the physical motion within the test sample modified the harmonic magnitudes. Work in [45] employed gold electrodes to probe suspensions of sediments, and found that gold electrodes can exhibit a considerable stabilization time. Additionally, cleaning and reinserting gold electrodes into the sediment suspension could alter the electrode half-cell potential (see Sec. 3.5) by hundreds of millivolts. The authors suggested a one hour stabilization period as being practical, as determined empirically. Mechanical disturbance of the electrode by the sediment was also considered as an influencing factor in instability, but the authors found quantifying this effect to be difficult.

Mechanical disturbance is a strong possibility in the yeast test cases. The quiescent yeast tends to settle out of suspension over time as can be determined visually. The active yeast suspension displays considerable physical movement within the sample. S. cerevisiae is a top-fermenting yeast, meaning that when metabolically active, the cells rise to the solution surface. As metabolic activity decreases the cells tend to settle out of suspension (see Sec. 4.3.3). While quantifying this mechanical disturbance is difficult, visual observations made of the active yeast indicated that yeast cells collected at the surface of the medium from hours one through six. After hour six the yeast cells began settling out of suspension, and by hour twelve the medium was visually less opaque with a slurry of cells on the container floor. While this analysis of cellular movement is not rigorous, it does provide a reference against which the harmonic variation can be correlated. The quiescent yeast trials, in which most of the settling occurred prior to data being recorded (a twenty minute settling time was allowed for these trials) show little variation in the harmonic magnitudes. The active yeast cases have a greater variation in harmonic magnitude in the early hours of the test. While the transmembrane protein effect afforded to the differences in 
harmonic magnitudes between the active and quiescent yeast cases in [3] may contribute to the observed harmonic patterns, mechanical disturbance cannot be discounted.

The second aspect to consider is the results of the reference spectrum method. The reference spectrum method provides a means of normalizing the quiescent and active response data to enable comparison with the results of [3]. The results of the reference spectrum method for both the quiescent and active cases did not agree with the results of [3]. One difficulty in applying this comparison is that only ten seconds of data are presented in [3]. Effects such as electrode instability, mechanical disturbance, as well as subtle differences in apparatus may be factors which account for the disagreement between results. As such, the work to follow in this dissertation will not focus on repeating the work of [3], but will instead employ a modified apparatus and different techniques to elucidate any nonlinear biological response.

While electrode instability and mechanical disturbance can contribute artifacts to the recorded data, electrode polarization effects must also be given careful consideration. Electrode processes can be highly nonlinear depending on the signal levels due to the underlying mechanism of electrode polarization (see Sec. 3.5.1). Examining the magnitude of the harmonics as compared to the fundamental in the electrolyte medium provide a means of gauging the nonlinearity of the electrode-electrolyte system. In the electrolyte medium of this work the strongest harmonic is approximately $20 \mathrm{~dB}$ down from the fundamental. This level of polarization-induced harmonics may serve to mask the biological signal of interest. Therefore the following experimental work will reevaluate the choice of electrode material to reduce electrode polarization and improve electrode stability.

The third aspect to consider is the results of the control experiments. The first control experiment examined the difference in spectral magnitudes between living and dead cells. 
Little difference was observed between living and dead quiescent cells in terms of relative magnitude of the harmonics. Killing a suspension of active cells led to a considerably different harmonic pattern than the living cells exhibited. This difference could be accounted for either by the lack of mechanical disturbance, or by the lack of a biological response occurring in the dead cells. Further work is required to determine the respective contributions of these two effects.

With the knowledge gained from this set of parallel experimental work, the following set of experimental work was undertaken to reduce electrode polarization artifacts and electrode instability to assist in revealing a transmembrane protein response.

\subsection{Ag-AgCl Electrode Experimental Work}

\subsubsection{Improvements}

The next major improvement to the NLDS apparatus was the replacement of the gold electrodes with $\mathrm{Ag}-\mathrm{AgCl}$ electrodes. $\mathrm{Ag}-\mathrm{AgCl}$ electrodes approach the nonpolarizable ideal, and as such should minimize polarization artifacts. Additionally, $\mathrm{Ag}-\mathrm{AgCl}$ electrodes have a more stable half-cell potential than gold [35]. Electrodes were prepared by chloriding 22 gauge (640 $\mu \mathrm{m}$ diameter) silver wire (99.99\% pure, A-M Systems, Sequim, Washington) in sodium hypochlorite for twelve hours at $22^{\circ} \mathrm{C}$. Preparation was done in batches to allow comparison of test cases using electrodes from the same batch.

Another modification which improved the apparatus was to reduce the diameter of the test container, and to decrease the sample size a corresponding amount. Sample sizes of 2 $\mathrm{mL}$ were pipetted into open glass cylinders with a diameter of $16 \mathrm{~mm}$. The electrode length was increased to $20 \mathrm{~mm}$, with $15 \mathrm{~mm}$ being immersed in the test sample. This allowed the electrodes to penetrate the test sample from the sample surface to a point $2 \mathrm{~mm}$ above the 
bottom of the test container. This was important in allowing the current to fully permeate the cellular material even when the settling of the cells occurred.

The total duration of all tests was set at twelve hours. From the results of Sec. 4.5.2 it was expected that no additional meaningful information would be obtained after the twelfth hour. To observe any initial stability due to mechanical disturbance the yeast suspensions were stirred immediately prior to the recording of data.

\subsubsection{Ag-AgCl Electrode Experimental Results}

Three trials were ran with data being recorded for both the drive and response channels. Each trial sampled data from the electrolyte test case, the quiescent yeast test case, and the active yeast test case in parallel. The plots in which these data are presented are grouped according to trial, as opposed to test case. This grouping was chosen to enable a visible assessment of harmonic patterns in the three test cases as the reference spectrum method will no longer be used. The following six plots, from Fig. 4.21 through Fig. 4.26 present the response and drive PSD harmonic magnitudes for the three trials.

\section{Reduction of Polarization Harmonics}

The first aspect to consider in the analysis of the plots of Fig. 4.21 through Fig. 4.26 is the reduction of harmonics in the electrolyte case as compared to the data of Sec. 4.5.2. To consolidate the data from Sec. 4.5.2 and the $\mathrm{Ag}-\mathrm{AgCl}$ data of this section Table 4.1 and Table 4.2 are provided for the response and drive data respectively. These tables contain the average of the harmonic magnitudes as well as the standard deviation for the three primary test cases of all trials. In calculating the values for these tables the first twenty minutes of

data for the $\mathrm{Ag}-\mathrm{AgCl}$ data were discarded so that the initial conditions in terms of settling 

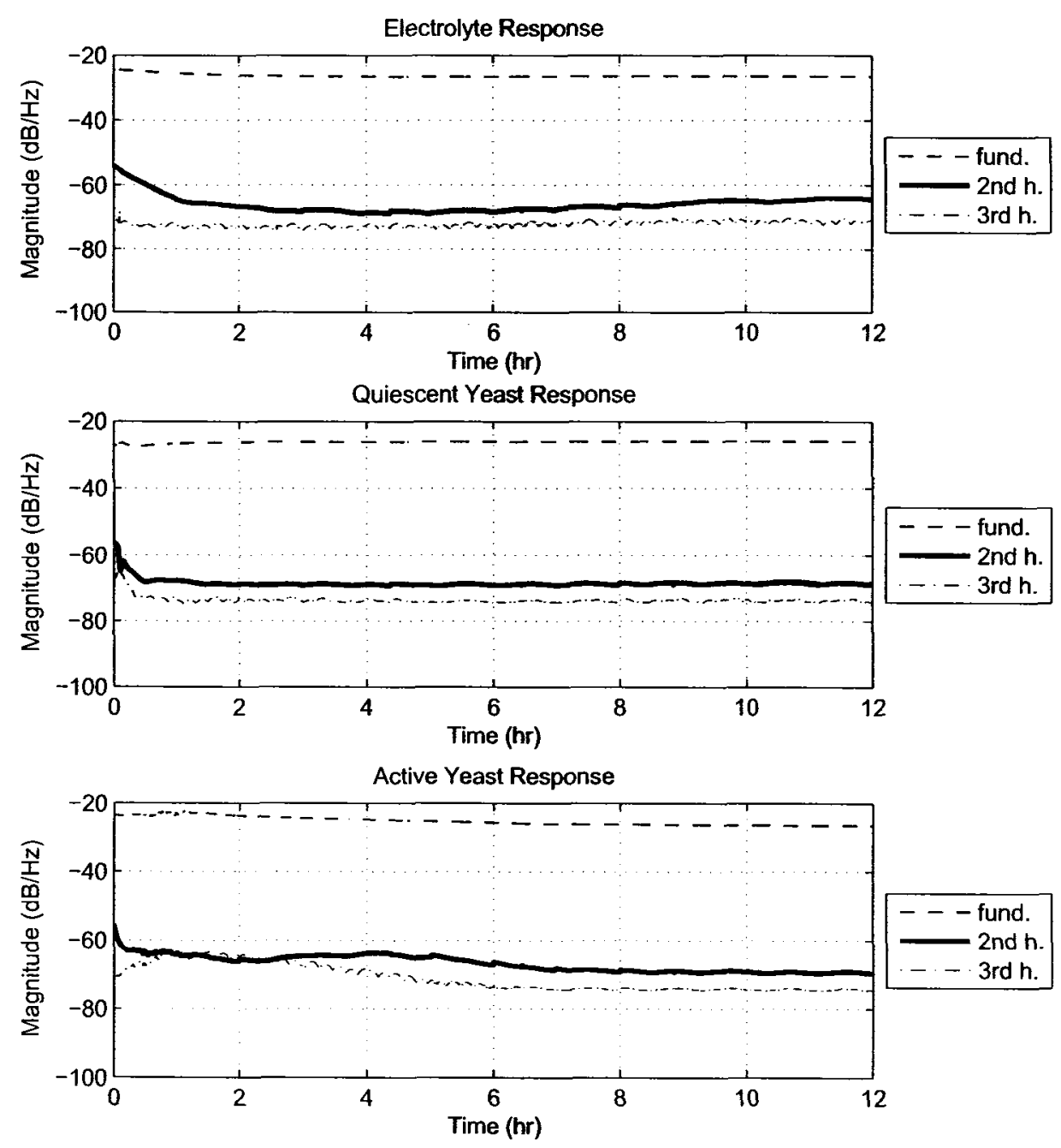

Figure 4.21: Trial 1 response time-course spectral results: Data were collected for the three primary test cases in parallel using $\mathrm{Ag}-\mathrm{AgCl}$ electrodes. The plots show the response PSD magnitudes of the fundamental, second harmonic and third harmonic for the electrolyte, quiescent, and active cases respectively. 

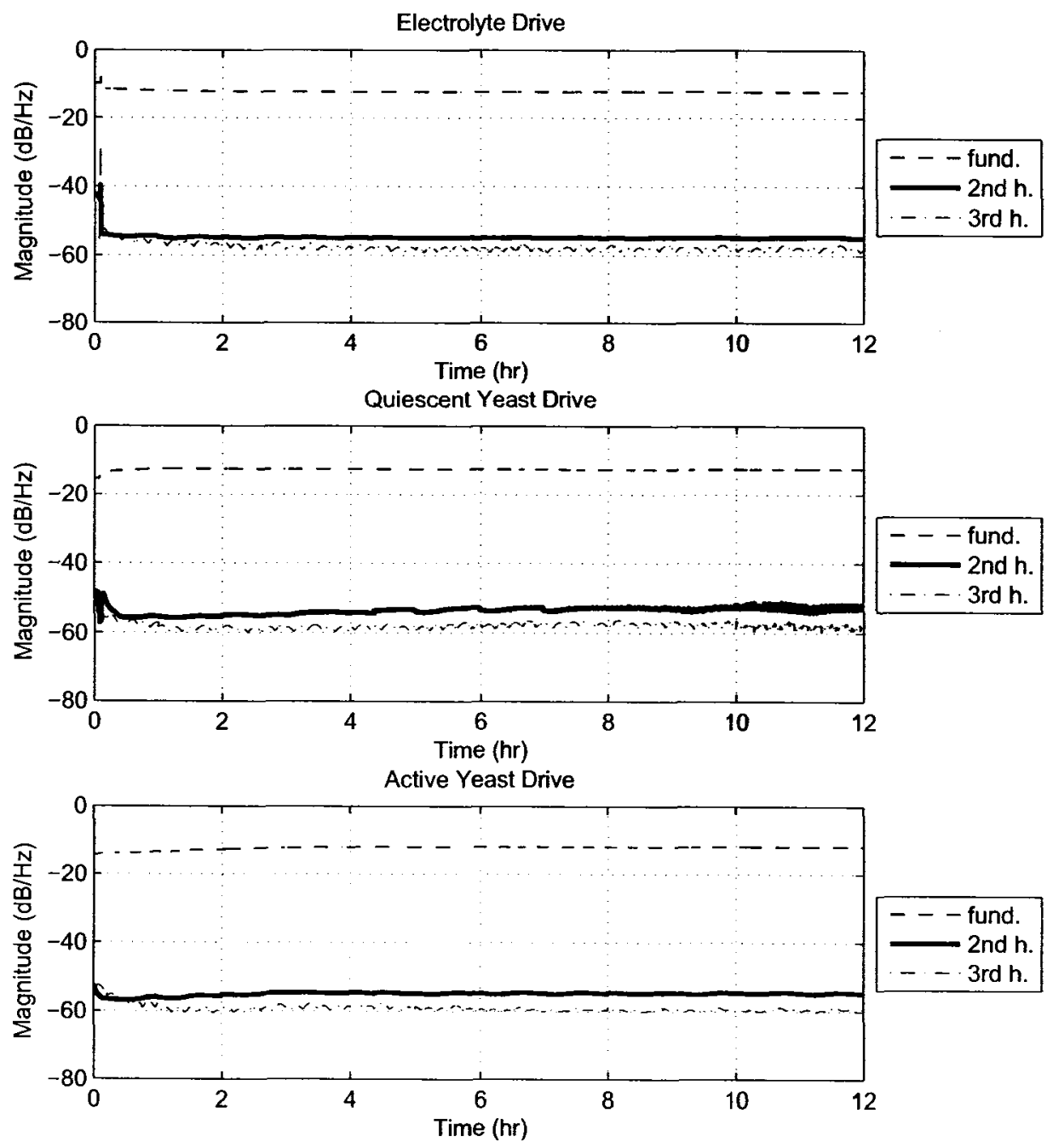

Figure 4.22: Trial 1 drive time-course spectral results: Data were collected for the three primary test cases in parallel using $\mathrm{Ag}-\mathrm{AgCl}$ electrodes. The plots show the drive PSD magnitudes of the fundamental, second harmonic and third harmonic for the electrolyte, quiescent, and active cases respectively. 

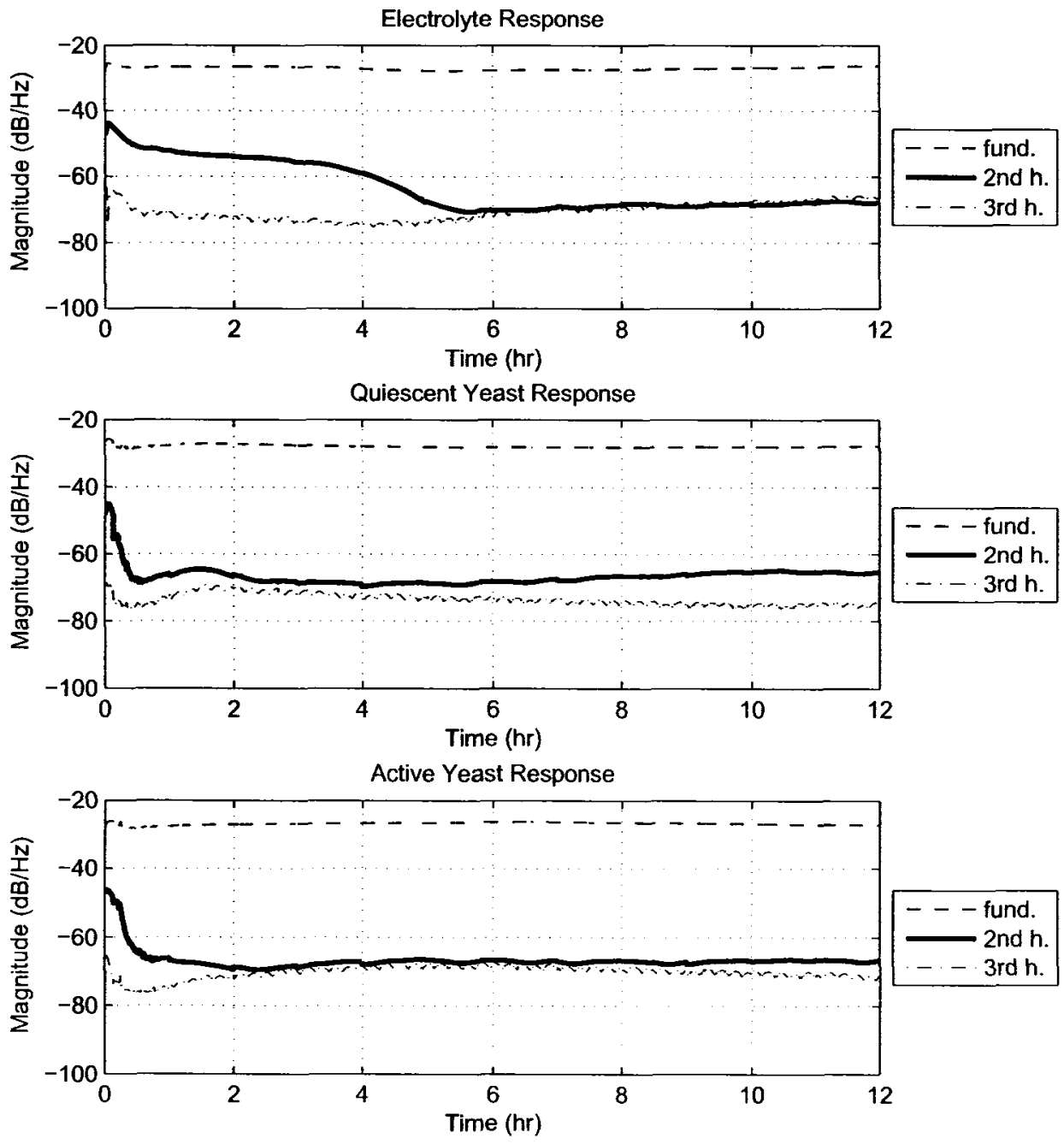

Figure 4.23: Trial 2 response time-course spectral results: Data were collected for the three primary test cases in parallel using $\mathrm{Ag}-\mathrm{AgCl}$ electrodes. The plots show the response PSD magnitudes of the fundamental, second harmonic and third harmonic for the electrolyte, quiescent, and active cases respectively. 

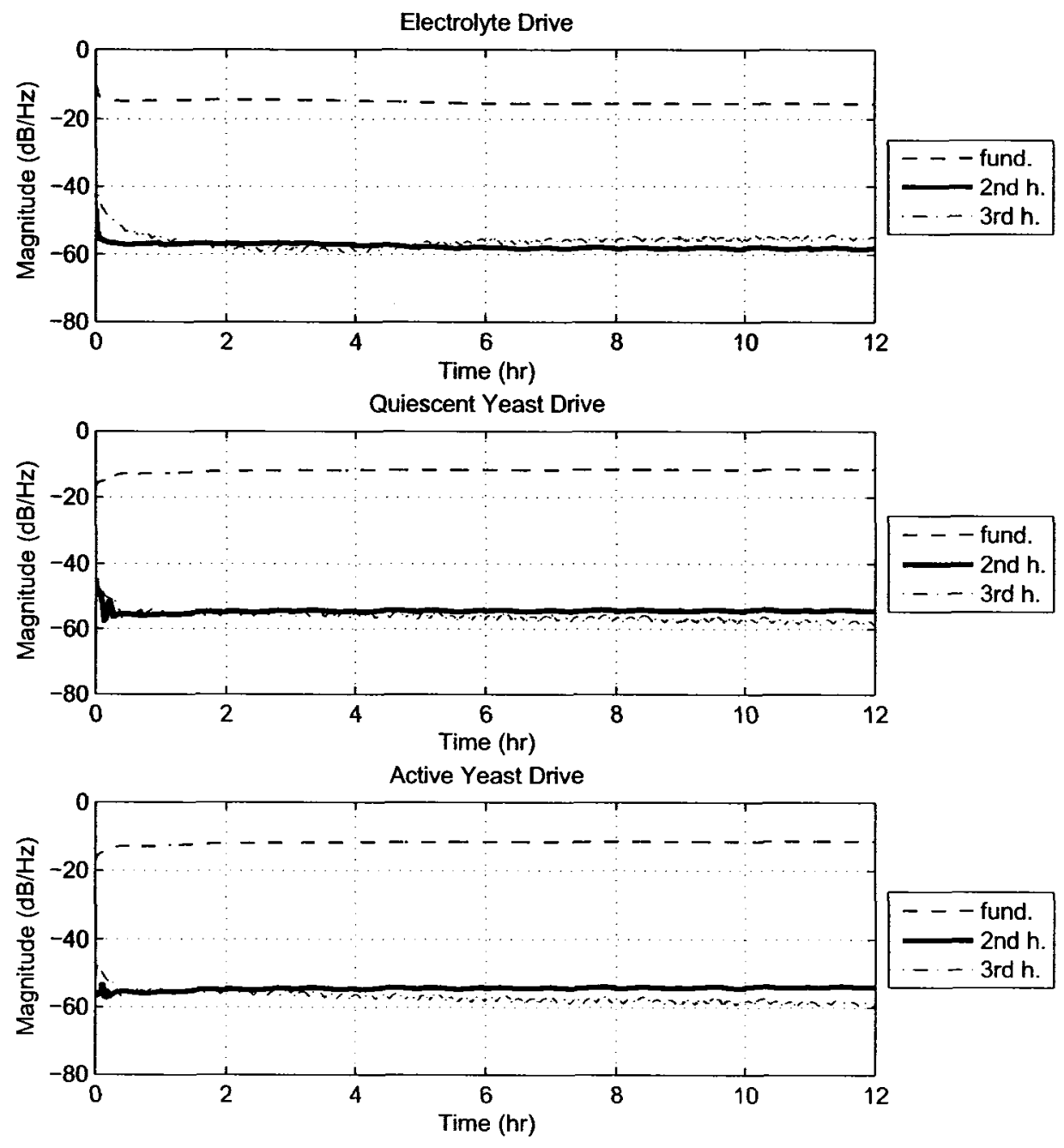

Figure 4.24: Trial 2 drive time-course spectral results: Data were collected for the three primary test cases in parallel using $\mathrm{Ag}-\mathrm{AgCl}$ electrodes. The plots show the drive PSD magnitudes of the fundamental, second harmonic and third harmonic for the electrolyte, quiescent, and active cases respectively. 

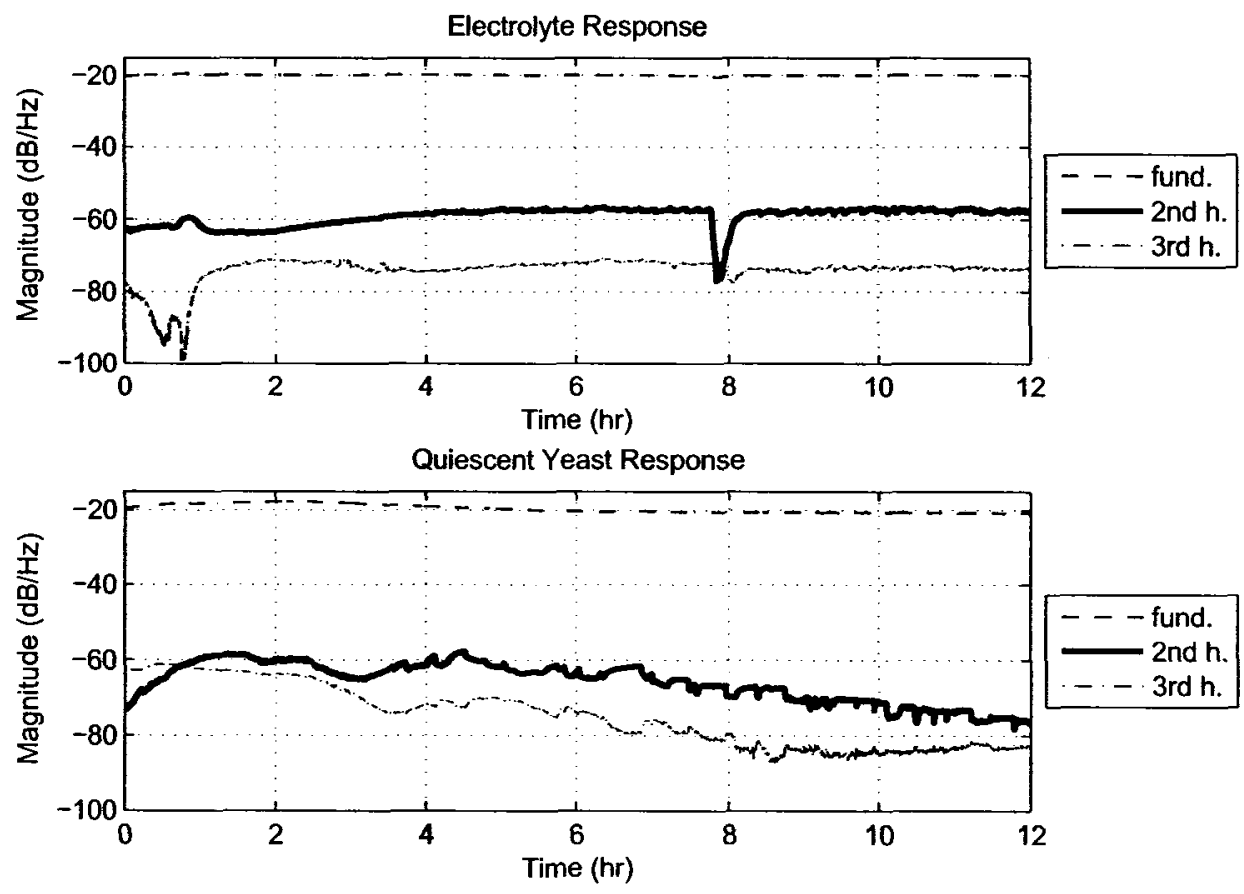

Active Yeast Response

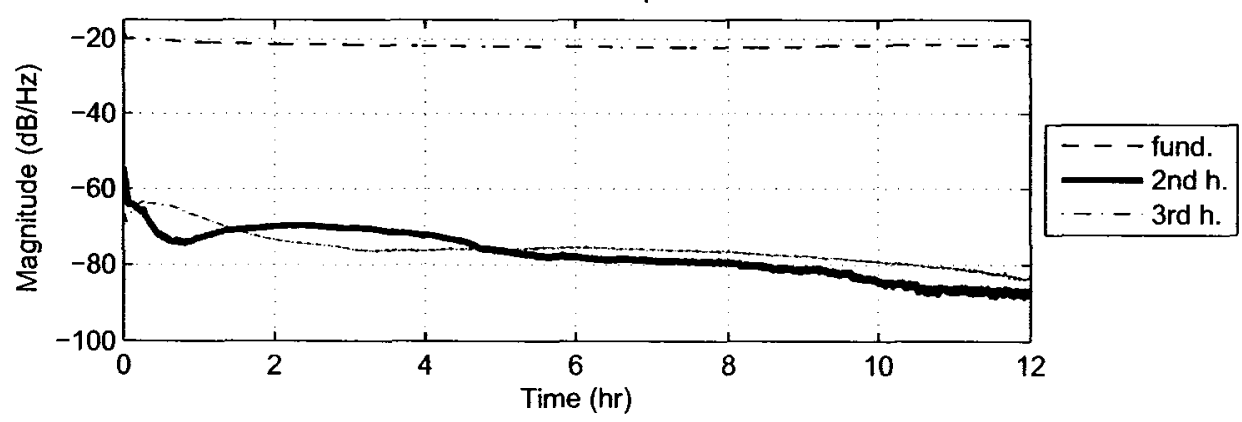

Figure 4.25: Trial 3 response time-course spectral results: Data were collected for the three primary test cases in parallel using $\mathrm{Ag}-\mathrm{AgCl}$ electrodes. The plots show the response PSD magnitudes of the fundamental, second harmonic and third harmonic for the electrolyte, quiescent, and active cases respectively. 


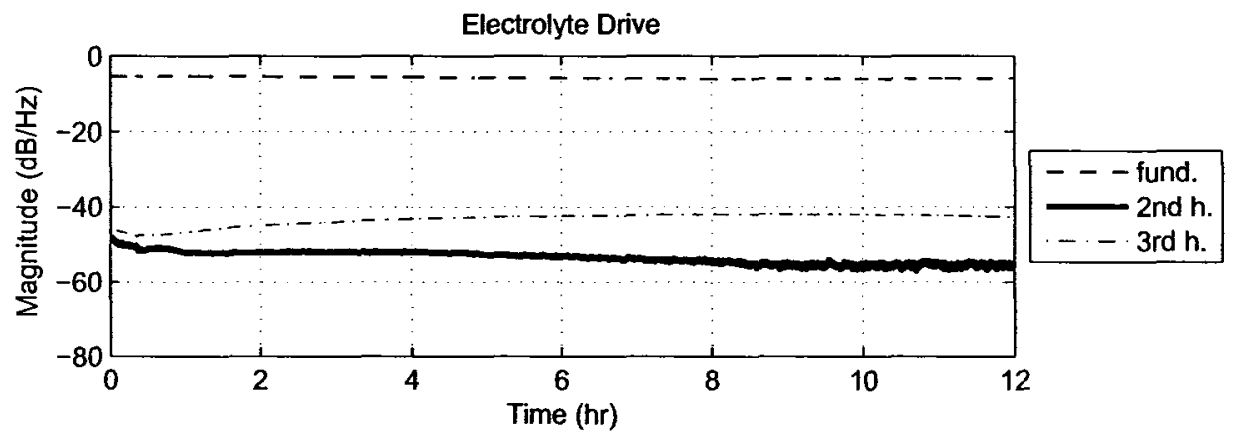

Quiescent Yeast Drive
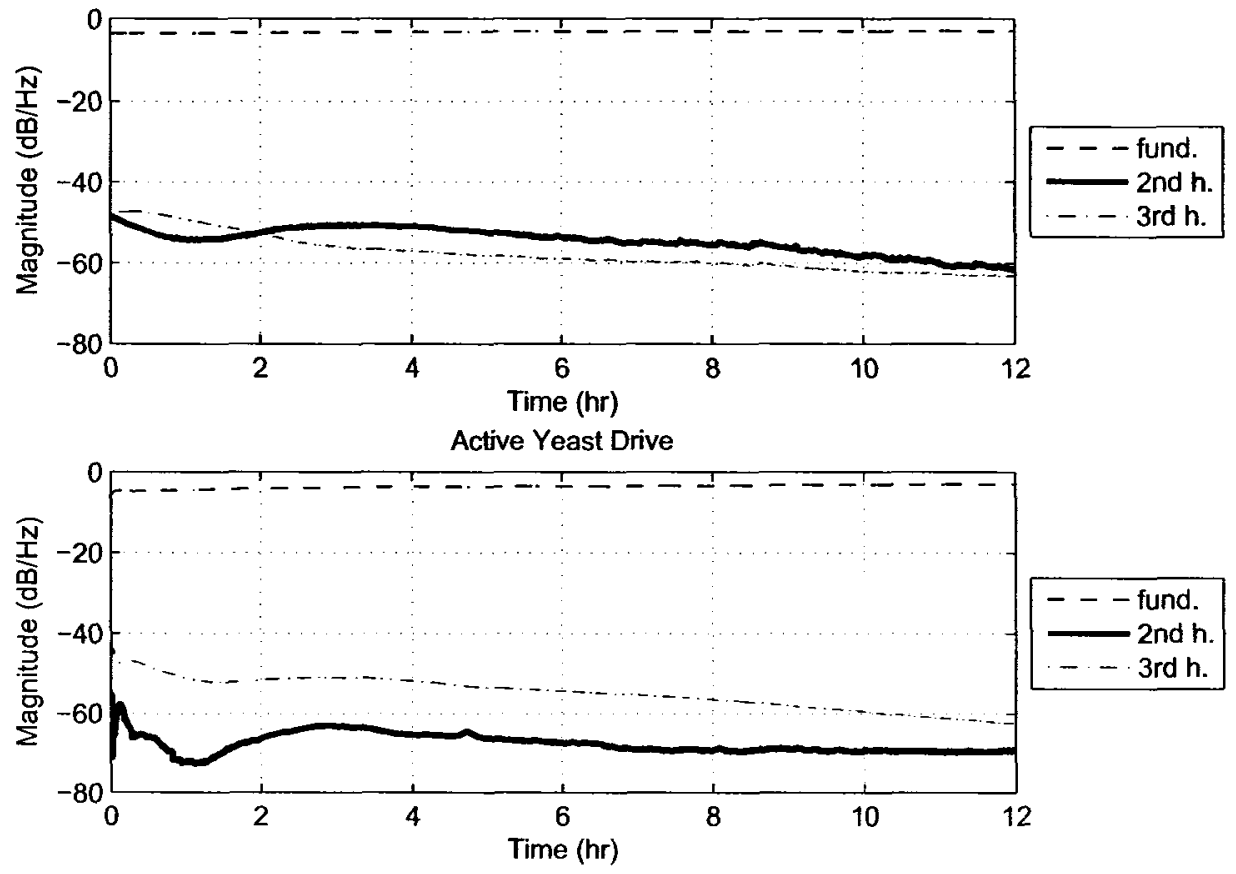

Figure 4.26: Trial 3 drive time-course spectral results: Data were collected for the three primary test cases in parallel using $\mathrm{Ag}-\mathrm{AgCl}$ electrodes. The plots show the drive PSD magnitudes of the fundamental, second harmonic and third harmonic for the electrolyte, quiescent, and active cases respectively. 
time would correspond with those of the gold data. The last four hours and twenty minutes of the gold data were discarded to enable comparison using the same time base as the $\mathrm{Ag}-\mathrm{AgCl}$ data.

Using values from Table 4.1, in the work of this section in which $\mathrm{Ag}-\mathrm{AgCl}$ electrodes were employed, the strongest harmonic is on average $38.5 \mathrm{~dB}$ down from the fundamental in the response signal of the electrolyte medium. The earlier work with gold electrodes found the strongest response harmonic to be approximately $18.5 \mathrm{~dB}$ down on average. Therefore, using $\mathrm{Ag}-\mathrm{AgCl}$ electrodes has improved the reduction of electrode polarization artifacts by approximately $20 \mathrm{~dB}$. This reduction is important in minimizing the effect of polarization artifacts which could mask any nonlinear transmembrane protein response.

\section{Electrode Stability}

The second aspect to consider is electrode stability. The fast time-base variation observed with the gold electrodes is not apparent with the $\mathrm{Ag}-\mathrm{AgCl}$ electrodes. The drift in the harmonic magnitudes which can be observed in the gold data also occurs in the $\mathrm{Ag}-\mathrm{AgCl}$ data. From Table 4.1, the means of the standard deviation of the fundamental, second harmonic, and third harmonic for the electrolyte response for the gold and $\mathrm{Ag}-\mathrm{AgCl}$ data are $1.97 \mathrm{~dB}$ and $2.21 \mathrm{~dB}$ respectively. The greater standard deviation of the $\mathrm{Ag}-\mathrm{AgCl}$ electrolyte response indicates that response electrode stability is marginally worse as compared to the gold electrodes. Applying the same analysis to the drive data of Table 4.2 a different outcome is obtained. The average of the standard deviations here are $2.42 \mathrm{~dB}$ and $0.775 \mathrm{~dB}$ for the gold and $\mathrm{Ag}-\mathrm{AgCl}$ respectively. This indicates that the effective current spectral components are considerably more stable with the $\mathrm{Ag}-\mathrm{AgCl}$ electrodes. It is likely that the absolute magnitude of the spectral components affects the standard deviation, and 
further work is required to understand this. Regardless of this, using $\mathrm{Ag}-\mathrm{AgCl}$ electrodes has reduced the polarization harmonics without compromising stability. As such, the data recorded in this section can be considered superior to that of Sec. 4.5 .2 in terms of nonlinear artifact reduction.

\section{Correlation with Metabolic Activity}

With a better understanding of the electrode properties in place, the third aspect to consider is if some harmonic pattern can be correlated with metabolic activity. It is first necessary to clarify the status of the biological samples over time. For the quiescent yeast two phases can defined:

- Phase 1 (hours 0 to 1 ): This phase, as determined visually, is characterized by the rapid settling out of suspension of the yeast cells.

- Phase 2 (hours 1 to 12 ): This phase is characterized by no metabolic activity, or very low levels of metabolism of endogenous energy stores.

For the active yeast three phases can be defined:

- Phase 1 (hours 0 to 1): This phase, as determined visually, is characterized by the rapid settling out of suspension of the yeast cells.

- Phase 2 (hours 1 to 5): This phase, as determined visually, is characterized by a high level of metabolic activity as glucose is consumed. Cells rise to the surface and the formation of gas bubbles is apparent.

- Phase 3 (hours 5 to 12): This phase, as determined visually, is characterized by lesser metabolic activity, with cells settling out of suspension. Glucose concentration measured at hour 12 of trial 3 was $25.5 \mathrm{mmol} \cdot \mathrm{L}^{-1}$, a decrease of $85 \%$. 


\begin{tabular}{|c|c|c|c|c|c|}
\hline \multicolumn{6}{|c|}{ Response Harmonic Statistics } \\
\hline & & \multicolumn{2}{|c|}{ Gold } & \multicolumn{2}{|c|}{$\mathrm{Ag}-\mathrm{AgCl}$} \\
\hline & & $\begin{array}{r}\text { Mean } \\
(\mathrm{dB})\end{array}$ & $\begin{array}{r}\text { St. Dev. } \\
\text { (dB) }\end{array}$ & $\begin{array}{r}\text { Mean } \\
(\mathrm{dB})\end{array}$ & $\begin{array}{r}\text { St. Dev. } \\
\text { (dB) }\end{array}$ \\
\hline \multirow{10}{*}{ Electrolyte } & Trial 1 fund. & -13.28 & 0.46 & -26.39 & 0.38 \\
\hline & Trial 2 fund. & -20.24 & 1.53 & -27.05 & 0.45 \\
\hline & Trial 3 fund. & -13.61 & 0.37 & -19.97 & 0.14 \\
\hline & Trial 1 2nd harm. & -31.49 & 2.49 & -66.29 & 1.93 \\
\hline & Trial 2 2nd harm. & -40.82 & 2.72 & -63.73 & 6.80 \\
\hline & Trial 3 2nd harm. & -30.33 & 0.78 & -58.90 & 2.70 \\
\hline & Trial 1 3rd harm. & -42.54 & 1.86 & -72.12 & 0.91 \\
\hline & Trial 2 3rd harm. & -51.98 & 1.03 & -70.58 & 2.57 \\
\hline & Trial 3 3rd harm. & -59.52 & 6.44 & -73.82 & 4.04 \\
\hline & Mean & -33.76 & 1.97 & -53.21 & 2.21 \\
\hline \multirow{10}{*}{ Quiescent } & Trial 1 fund. & $-10.5 \overline{3}$ & 0.25 & -26.18 & 0.28 \\
\hline & Trial 2 fund. & -12.22 & 1.18 & -27.95 & 0.30 \\
\hline & Trial 3 fund. & -13.79 & 0.34 & -19.69 & 1.12 \\
\hline & Trial 1 2nd harm. & -34.70 & 0.93 & -68.59 & 0.40 \\
\hline & Trial 2 2nd harm. & -39.75 & 1.71 & -67.05 & 1.45 \\
\hline & Trial 3 2nd harm. & -36.76 & 0.90 & -65.65 & 5.23 \\
\hline & Trial 1 3rd harm. & -44.16 & 1.18 & -73.76 & 0.39 \\
\hline & Trial 2 3rd harm. & -45.62 & 1.03 & -73.49 & 1.70 \\
\hline & Trial 3 3rd harm. & -48.39 & 2.19 & -75.08 & 7.96 \\
\hline & Mean & -31.77 & 1.08 & -55.27 & 2.09 \\
\hline \multirow{10}{*}{ Active } & Trial 1 fund. & -14.94 & 0.86 & -25.36 & 1.21 \\
\hline & Trial 2 fund. & -46.00 & 1.59 & -72.47 & 4.29 \\
\hline & Trial 3 fund. & -48.89 & 1.69 & -76.14 & 4.71 \\
\hline & Trial 1 2nd harm. & -51.78 & 1.78 & -79.81 & 5.12 \\
\hline & Trial 2 2nd harm. & -54.67 & 1.88 & -83.48 & 5.54 \\
\hline & Trial 3 2nd harm. & -57.57 & 1.98 & -87.15 & 5.95 \\
\hline & Trial 13 rd harm. & -60.46 & 2.07 & -90.82 & 6.37 \\
\hline & Trial 2 3rd harm. & -63.35 & 2.17 & -94.49 & 6.79 \\
\hline & Trial 3 3rd harm. & -66.25 & 2.27 & -98.16 & 7.20 \\
\hline & Mean & -51.55 & 1.81 & -78.65 & 5.24 \\
\hline
\end{tabular}

Table 4.1: Response harmonics means and standard deviations: The mean and standard deviation of the response spectral components for both the gold and $\mathrm{Ag}-\mathrm{AgCl}$ electrodes are presented in this table. This enables comparing electrode performance for the test cases. 


\begin{tabular}{|c|c|c|c|c|c|}
\hline \multicolumn{6}{|c|}{ Drive Harmonic Statistics } \\
\hline & & \multicolumn{2}{|c|}{ Gold } & \multicolumn{2}{|c|}{$\mathrm{Ag}-\mathrm{AgCl}$} \\
\hline & & $\begin{array}{r}\text { Mean } \\
(\mathrm{dB}) \\
\end{array}$ & $\begin{array}{r}\text { St. Dev. } \\
\text { (dB) }\end{array}$ & $\begin{array}{r}\text { Mean } \\
(\mathrm{dB}) \\
\end{array}$ & $\begin{array}{r}\text { St. Dev. } \\
\text { (dB) }\end{array}$ \\
\hline \multirow{10}{*}{ Electrolyte } & Trial 1 fund. & -32.62 & 1.09 & -12.20 & 0.14 \\
\hline & Trial 2 fund. & -32.79 & 1.53 & -15.09 & 0.48 \\
\hline & Trial 3 fund. & -18.19 & 0.53 & -5.72 & 0.23 \\
\hline & Trial 1 2nd harm. & -58.55 & 2.84 & -54.77 & 0.14 \\
\hline & Trial 2 2nd harm. & -68.25 & 5.44 & -57.73 & 0.61 \\
\hline & Trial 3 2nd harm. & -54.92 & 2.62 & -53.50 & 1.55 \\
\hline & Trial 1 3rd harm. & -55.74 & 1.31 & -57.55 & 0.87 \\
\hline & Trial 2 3rd harm. & -58.82 & 3.60 & -56.27 & 1.45 \\
\hline & Trial 3 3rd harm. & -46.74 & 2.79 & -43.23 & 1.50 \\
\hline & Mean & -47.40 & 2.42 & -39.56 & 0.78 \\
\hline \multirow{10}{*}{ Quiescent } & Trial 1 fund. & -31.95 & 1.92 & -12.68 & 0.09 \\
\hline & Trial 2 fund. & -33.55 & 1.75 & -11.82 & 0.34 \\
\hline & Trial 3 fund. & -30.12 & 0.68 & -3.14 & 0.15 \\
\hline & Trial 1 2nd harm. & -76.62 & 4.04 & -53.58 & 1.06 \\
\hline & Trial 2 2nd harm. & -72.07 & 3.49 & -54.62 & 0.39 \\
\hline & Trial 3 2nd harm. & -64.20 & 2.84 & -54.60 & 3.04 \\
\hline & Trial 1 3rd harm. & -55.14 & 2.23 & -57.90 & 0.82 \\
\hline & Trial 2 3rd harm. & -54.76 & 1.50 & -56.25 & 1.06 \\
\hline & Trial 3 3rd harm. & -46.37 & 1.18 & -57.95 & 4.14 \\
\hline & Mean & -51.64 & 2.18 & -40.28 & 1.23 \\
\hline \multirow{10}{*}{ Active } & Trial 1 fund. & -29.01 & 2.06 & $-12 . \overline{16}$ & 0.53 \\
\hline & Trial 2 fund. & -32.26 & 2.08 & -11.68 & 0.39 \\
\hline & Trial 3 fund. & -31.14 & 2.05 & -3.45 & 0.44 \\
\hline & Trial 1 2nd harm. & -62.87 & 2.00 & -54.89 & 0.57 \\
\hline & Trial 2 2nd harm. & -65.82 & 3.55 & -54.43 & 0.46 \\
\hline & Trial 3 2nd harm. & -66.20 & 3.84 & -67.64 & 2.27 \\
\hline & Trial 1 3rd harm. & -55.50 & 4.04 & -59.18 & 0.67 \\
\hline & Trial 2 3rd harm. & -57.90 & 1.86 & -57.17 & 1.32 \\
\hline & Trial 3 3rd harm. & -66.20 & 3.77 & -55.06 & 3.72 \\
\hline & Mean & -51.88 & 2.81 & -41.74 & 1.15 \\
\hline
\end{tabular}

Table 4.2: Drive harmonics means and standard deviations: The mean and standard deviation of the drive spectral components for both the gold and $\mathrm{Ag}-\mathrm{AgCl}$ electrodes are presented in this table. This enables comparing electrode performance for the test cases. 
By applying a value of one to represent the period of maximum metabolic activity, and a value of zero to represent lesser or no metabolic activity in a test case, a model roughly approximating the metabolic activity over time can be formed. The step function model for the active yeast, shown in Fig. 4.27 can then be correlated with the magnitude of the spectral components. This approach provides a method of obtaining a numerical relationship between the harmonic magnitudes and the visually determined metabolic activity.

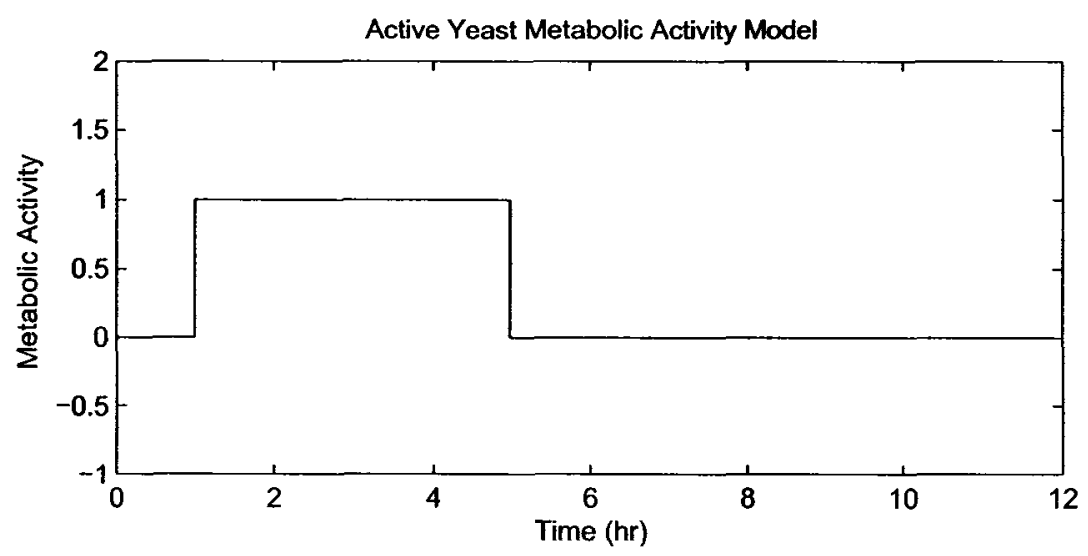

Figure 4.27: Metabolic activity step function model: To enable correlating harmonic magnitudes with metabolic activity, a step function model of metabolic activity was formed. The active yeast displayed strong metabolic activity over hours one to five.

This correlation between the metabolic activity model and the spectral magnitudes was applied for the three trials using tools available in the MATLAB environment. Active yeast data were correlated with the data of Fig. 4.27. The results for both the gold and $\mathrm{Ag}-\mathrm{AgCl}$ response electrode data are presented in Table 4.3. According to the plots of [3] the second and third harmonics show an increase when metabolically active. Therefore the correlation for the second and third harmonics in the active case should be positive and large. As a control the quiescent harmonics were also correlated to the active model. Ideally there should be little correlation in the quiescent case. 
By examining the correlation coefficients of Table 4.3 some conclusions can be drawn. The gold electrode data show a predominant negative correlation in the active case. This is counter to what was expected, but indicates that some effect can be observed. For the quiescent case, the correlation is considerably smaller. It can be concluded that the gold electrode data are correlated with the step function metabolic model, but not in the manner that was expected. For the $\mathrm{Ag}-\mathrm{AgCl}$ electrode data, the active case shows some positive correlation in two of the cases. Unfortunately the quiescent case, being used as a control here, displays approximately the same level of correlation. Thus the step function model is not correlated with the $\mathrm{Ag}-\mathrm{AgCl}$ data. The harmonic patterns in the $\mathrm{Ag}-\mathrm{AgCl}$ response plots show a different pattern between the active and quiescent cases when examined by eye. It is therefore likely that the model used in the correlation is too simplistic to reveal the relationship between harmonic magnitude and metabolic activity.

\begin{tabular}{|c|l|r|r|}
\hline \multicolumn{4}{|c|}{ Response Correlation Coefficients } \\
\hline \multirow{4}{*}{ Quiescent (Control) } & Gold & Ag-AgCl \\
\hline & Trial 1 2nd harm. & 0.37 & -0.21 \\
& Trial 1 3rd harm. & 0.34 & -0.11 \\
& Trial 2 2nd harm. & 0.30 & -0.24 \\
& Trial 2 3rd harm. & 0.61 & 0.77 \\
& Trial 3 2nd harm. & 0.35 & 0.65 \\
& Trial 3 3rd harm. & 0.19 & 0.59 \\
\hline \multirow{5}{*}{ Active } & Trial 1 2nd harm. & -0.62 & 0.59 \\
& Trial 1 3rd harm. & -0.59 & 0.74 \\
& Trial 2 2nd harm. & -0.78 & -0.28 \\
& Trial 2 3rd harm. & -0.57 & -0.01 \\
& Trial 3 2nd harm. & 0.06 & 0.67 \\
& Trial 3 3rd harm. & -0.39 & 0.22 \\
\hline
\end{tabular}

Table 4.3: Correlation coefficients: The second and third response harmonics were correlated with the step function model of metabolic activity. 


\section{Fundamental Frequency}

The fourth aspect to consider in this set of experimental work is the observations made of the spectral component at the fundamental frequency. While repeatability across experimental runs was difficult to achieve when considering the second and third harmonics, the general behavior of the fundamental for the $\mathrm{Ag}-\mathrm{AgCl}$ data was repeatable. This section will examine the behavior of the fundamental using the data of trial 3 as representative of the general behavior.

The plots of Fig. 4.28 show the magnitude of the fundamental for the three primary test cases for which the harmonic magnitudes were shown earlier in Figure 4.25. Each case exhibits a different time-course behavior. The electrolyte fundamental remains fairly constant over the twelve hour period, other than in the first hour. The quiescent case is characterized by a rise and fall of approximately $2 \mathrm{~dB}$ over the first six hours, after which the fundamental remains constant. Finally, the active case exhibits a decrease of approximately $2 \mathrm{~dB}$ over the twelve hours.

The variation in the fundamental magnitude of the PSD is an interesting and repeatable characteristic that will be examined in more detail in this thesis. As a first step in understanding this variation, the drive current PSD fundamental is shown in Fig.4.29. From this plot it can be seen that effective current at the fundamental frequency increases the most in the active yeast case, increases a small amount in the resting yeast case, and decreases a small amount in the medium alone. Since current is proportional to conductivity when the system is approximated as linear, it appears that conductivity is changing over time, with a significant increase in the active case. This effect will be analyzed further in Chapter 5 . 


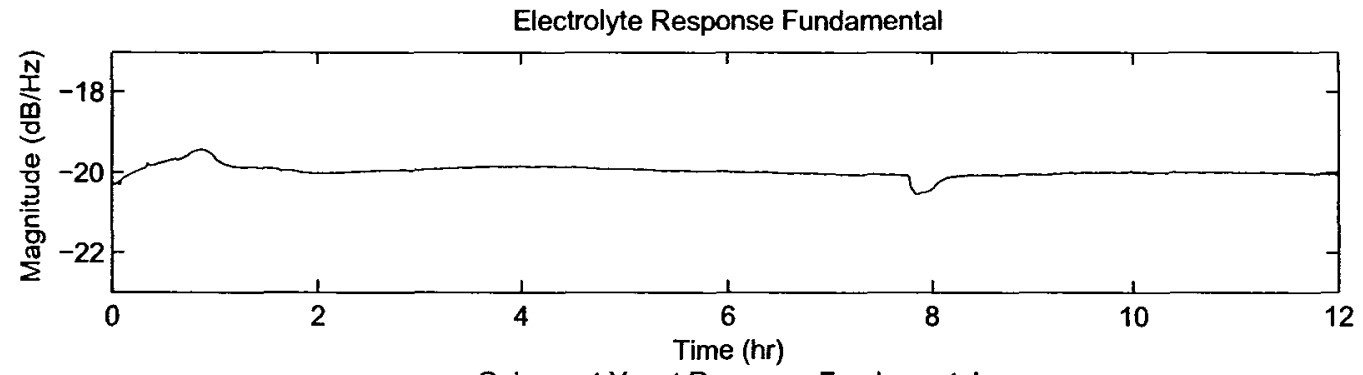

Quiescent Yeast Response Fundamental
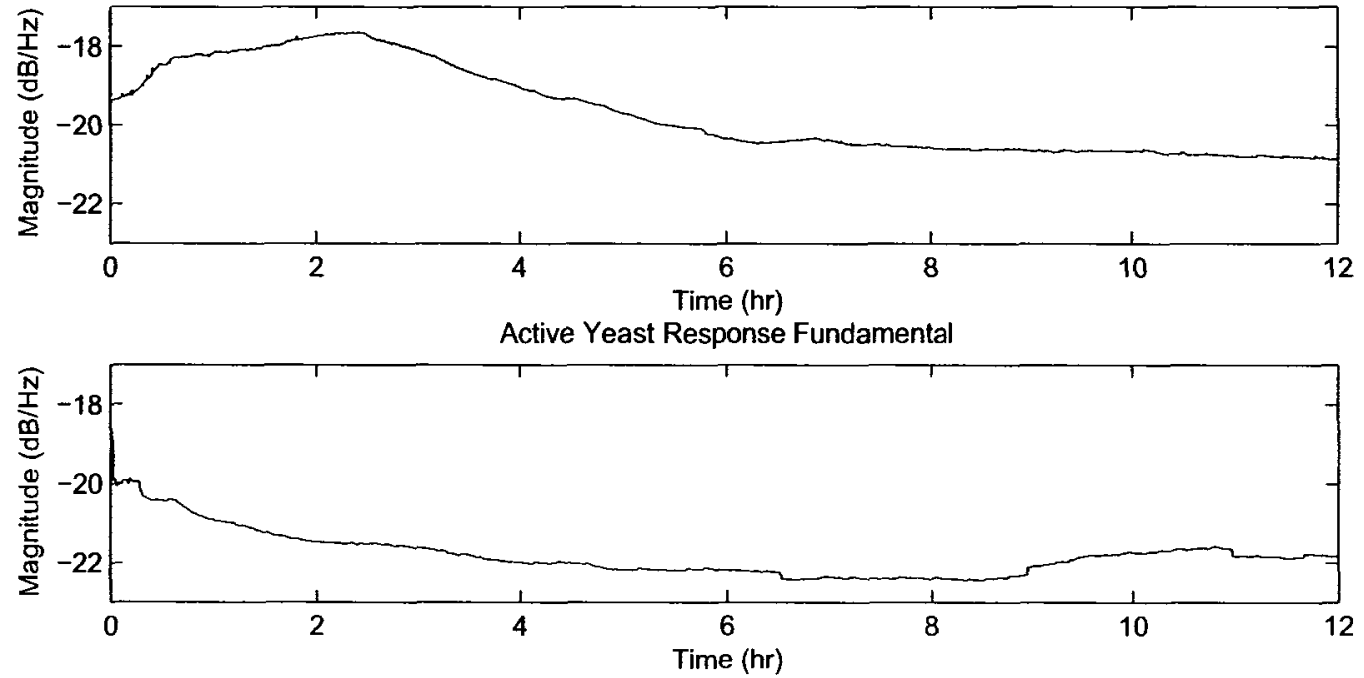

Figure 4.28: Trial 3 time-course response fundamental: The plots show the magnitude of the response PSD fundamental for the electrolyte, quiescent, and active cases respectively. 


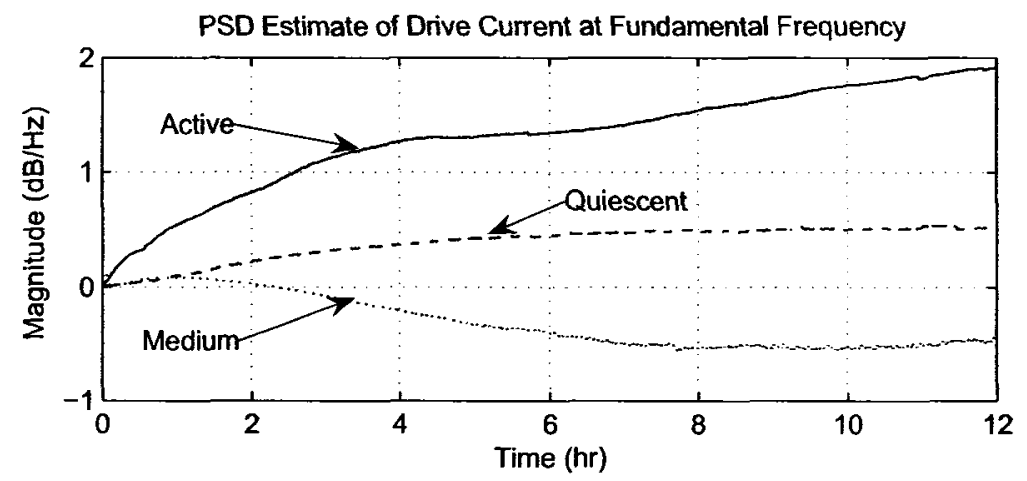

Figure 4.29: Trial 3 time-course drive current fundamental: The voltage across the series resistor $R_{m}$ was sampled to obtain a signal proportional to effective current. The PSD was normalized to account for small variations in initial conductivity. The results show that effective current over time differs between the active yeast, quiescent yeast and electrolyte medium.

\subsubsection{Discussion of Results}

The change of electrode material from gold to $\mathrm{Ag}-\mathrm{AgCl}$ improved the quality of data in terms of reducing nonlinear electrode polarization artifacts. In the experimental work of Section 4.5.2, in which gold electrodes were used, the second harmonic of the electrolyte case was only $18.5 \mathrm{~dB}$ below the fundamental. The improved $\mathrm{Ag}-\mathrm{AgCl}$ electrode apparatus showed the second harmonic of the electrolyte case to be $38.5 \mathrm{~dB}$ below the fundamental. The reduction of nonlinearity in the electrolyte when using the $\mathrm{Ag}-\mathrm{AgCl}$ electrodes indicates that polarization effects are reduced with this material.

While this work indicates that there is a different harmonic signature between the quiescent and active cases, results obtained with this modified experimental apparatus did not agree with [3]. In [3], odd harmonics dominated in the quiescent case, while even harmonics dominated in the active case. Again, results in this last set of experiments did not show this. This difference may be explained by the fact that a different experimental apparatus with $\mathrm{Ag}-\mathrm{AgCl}$ electrodes, rather than gold electrodes, was used. Also, a different size 
sample and test cell were used in these experiments.

Ambient temperature and conditions were not tightly controlled across the different trials and could therefore vary somewhat between the repeated experiments. This may have had some effect on the variation seen between the trials.

\subsection{Chapter Summary}

This chapter discussed the experimental apparatus and protocol used to apply the technique of NLDS to a suspension of the yeast $S$. cereviaiae, and then presented the results that were obtained. Three primary test cases were considered. The first of these was the electrolyte solution, which acts as the medium for the tests containing biological cells. The second test case was the quiescent yeast suspension, which was metabolically inactive. The third primary test cases was the active yeast suspension, which had a metabolizable glucose source.

The sequential and parallel experimental work employed gold electrodes and was performed with the goal of repeating part of the work of [3]. Results obtained did not agree with those of [3]. The nonlinearity due to electrode polarization was observed to be large, occurring at $18.5 \mathrm{~dB}$ down from the fundamental.

To reduce the observed polarization effects, a third set of experiments was run using $\mathrm{Ag}-\mathrm{AgCl}$ electrodes, a type of electrodes that approach the nonpolarizable ideal. Results obtained using these electrodes differed from the results acquired with gold electrodes and revealed a different harmonic pattern between the three primary test cases. Repeatability of harmonic patterns was difficult to achieve between experimental trials, but each of the three parallel test cases displayed a different harmonic pattern. This implies that the prediction of glucose concentration may be possible amongst one experimental data set, but will be 
difficult amongst different trials. The considerable difference in results between the data of the gold electrodes and that of the $\mathrm{Ag}-\mathrm{AgCl}$ electrodes indicates that electrode effects may be masking the transmembrane protein response that is of interest. This will be considered in detail in the following chapter.

The current monitoring resistor used in this apparatus was a novel addition to the NLDS system. This modification enabled the characterization of the effective current, and provided significant data. Effective current was found to differ between the three primary test cases, with a significant increase in the active case.

Before answering the questions posed at the beginning of this chapter, further analysis of the results, as well as additional experimentation, is required. This additional work will be presented in the following chapters. 


\section{Chapter 5}

\section{Analysis of Experimental Results}

\subsection{Overview}

One objective of the NLDS work of this thesis is to correlate a nonlinear biological response with metabolic activity. To do this, an understanding of how the electrode-electrolyte system can introduce artifacts into the recorded data is necessary. Without an understanding of system artifacts, there is the possibility that metabolic activity will be correlated with nonlinear system artifacts, rather than with the biological response of interest.

Electrode polarization effects are known to introduce nonlinearity into electrode-electrolyte systems under some conditions [6]. As such, it is particularly important to understand the nonlinear behavior of electrode polarization and how it can introduce artifacts in the data.

This section first presents a linearized circuit model of the electrode-electrolyte system. Linearization enables modelling the system using passive linear circuit components, simplifying analysis. The consequences of applying this linear simplification are then considered.

With a suitable model formed, experimental data from two different electrode geometries will then be examined. This will provide information about the linearity of the elec- 
trolyte bulk, and about nonlinear artifacts arising from the polarization impedance at the electrode-electrolyte interface. In particular, the effect that the addition of yeast to the electrolyte has on polarization impedance is analyzed.

Next, the effect of metabolic activity is considered as another area where artifacts may be induced. The average power over time is analyzed to obtain information about variation in conductivity for the electrolyte, quiescent, and active cases.

A hypothesis is then presented to account for the change in conductivity, and an analysis of how current variation affects harmonic magnitude is done. Finally, the physical effect of air bubbles in a suspension of quiescent yeast will be examined to simulate the physical effect of metabolic byproduct gases on the harmonic response.

\subsection{Circuit Model}

\subsubsection{Electrode Model}

The experimental results for the electrolyte case revealed nonlinearities in the response signal. Because there is no biological material present, the nonlinearities can be due to either electrode effects or to electrolyte effects, or a combination of the two. It is important to understand the nonlinear contribution of the electrode-electrolyte system when interpreting the biological response. Therefore, a more thorough analysis of the electrolyte case is required. This begins with an examination of electrode polarization.

In the background work of this thesis, some discussion of electrode polarization was given in Sec. 3.5.1. In this previous discussion, electrode polarization was described as consisting of three overpotentials in series, and encompassed the complete electrodeelectrolyte system. The three overpotentials making up the total electrode polarization result from series impedances in the electrode-electrolyte system [34]. Therefore the total 
electrode polarization impedance, denoted by $Z_{p}$, is:

$$
Z_{p}=R_{o}+Z_{c}+Z_{a}
$$

where $R_{o}$ is the ohmic overpotential due to the electrolyte bulk. $Z_{c}$ and $Z_{a}$ are the concentration overpotential impedance and activation energy overpotential impedance respectively. Both $Z_{c}$ and $Z_{a}$ occur at the electrode-electrolyte boundary and are therefore referred to as interfacial polarization impedance [36]. An interfacial polarization impedance term $Z_{i}$ can be defined as

$$
Z_{i}=Z_{c}+Z_{a}
$$

$R_{o}$ can be considered linear below a very high applied electric field threshold [35]. $Z_{i}$ is a function of current flow across the interface and is therefore nonlinear [35]. It is expected then that $Z_{i}$ will contribute some nonlinear artifacts to the system, and that $R_{o}$ could as well.

In the four electrode configuration, the assumption can be made that electrode polarization overpotentials are negligible in the recording electrodes. These electrodes are in series with a high impedance amplifier (according to the instrument specifications, an input impedance of $100 \mathrm{G} \Omega \| 50 \mathrm{pF}$ ) so current flow in the recording pair is negligible, and is due only to leakage currents. The recording electrodes are therefore assumed to be not externally polarized [35], and are therefore transparent.

To include electrode-electrolyte effects in the drive circuit, a Warburg equivalent circuit model of the electrode can be included. This electrode equivalent circuit, shown in Fig. 5.1 consists of the electrode half-cell potential $E_{h c}$, the parallel capacitance and resistance of the double layer of charge at the electrode-electrolyte interface $C_{d}$ and $R_{d}$, and the series resistance $R_{s}$ of the interface and the electrolyte bulk [34]. The half-cell potential $E_{h c}$ 
describes the potential difference between the electrode-electrolyte interface and the bulk of the electrolyte solution when no current is flowing. $E_{h c}$ is relatively stable for $\mathrm{Ag}-\mathrm{AgCl}$ electrodes as compared to gold.

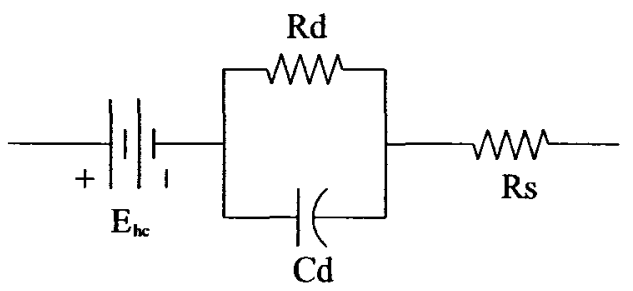

Figure 5.1: Warburg electrode equivalent circuit: The electrode half-cell potential is denoted by $E_{h c}$. The parallel capacitance and resistance of the double layer of charge at the electrode-electrolyte interface are denoted by $C_{d}$ and $R_{d}$. The series resistance of the interface and the electrolyte bulk is denoted by $R_{s}$.

At the low frequencies employed in the experimental work, $C_{d}$ will be assumed to be an open-circuit and can be neglected. The two series resistances $R_{s}$ and $R_{d}$ can then be separated into a series resistance due to the interface, denoted by $R_{i}$, and the series resistance of the electrolyte bulk, noted earlier as the ohmic polarization impedance $R_{o}$. The interfacial polarization impedance $Z_{i}$, now considered as purely resistive at low frequencies is transformed to $R_{i}$.

As mentioned earlier $R_{i}$ is well known to exhibit a nonlinear I-V relationship. A plot of the general I-V relationship of $R_{i}$ under $\mathrm{DC}$ conditions for $\mathrm{Ag}-\mathrm{AgCl}$ electrodes is shown in Fig. 5.2, as illustrated in [35]. With no current flowing, the half-cell potential $E_{h c}$ is observed. Any small deviation from $E_{h c}$, achieved by applying a DC voltage across the electrodes, results in a substantial current flow. An applied AC voltage would be superimposed on the DC bias, resulting in a nonlinear current signal. In the experimental work, no additional DC bias voltage is applied as a pure sinusoidal voltage source is used. The low frequency of $17 \mathrm{~Hz}$ used in the experimental work does suggest that some DC-like behavior could be observed in the system. This would cause the DC bias point to deviate from the 
$E_{h c}$ point, into a more nonlinear portion of the resistance curve. It is also apparent from this plot that the larger an applied AC signal is, the greater the nonlinear behavior will be.

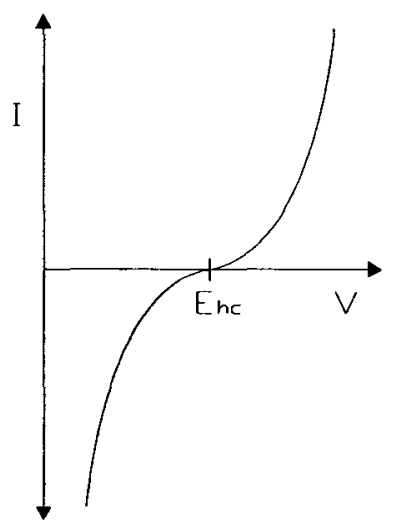

Figure 5.2: DC interfacial impedance I-V curve: The nonlinear nature of the interfacial impedance is illustrated by the $\mathrm{I}-\mathrm{V}$ relationship of an $\mathrm{Ag}-\mathrm{AgCl}$ electrode in a $0.9 \% \mathrm{NaCl}$ solution [35].

\subsubsection{Drive Circuit Model}

Incorporating the modified electrode equivalent circuit in to the drive circuit of the experimental apparatus yields the circuit of Fig. 5.3. In this model $E_{h c}$ is omitted to reflect the AC nature of the input voltage.

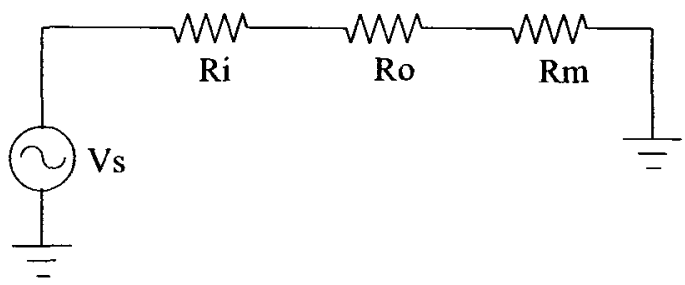

Figure 5.3: Drive circuit: $R_{i}$ is the interfacial polarization impedance, while $R_{o}$ is the ohmic polarization impedance due to the electrolyte bulk. The external current monitoring resistor is denoted by $R_{m}$.

Analyzing circuit behavior requires obtaining data from a set of the accessible terminals 
of the circuit. Accessible terminals are found at each electrode, and at $R_{m}$. The voltage signal recorded across $R_{m}$ enables calculating effective current, which along with the voltage signal across the recording electrodes, enables calculating the resistivity of the electrolyte. Data obtained from these two signals can then be used to solve the circuit parameters. Due to the nonlinear behavior of the circuit it is necessary to understand the implications of applying Ohm's law in solving circuit parameters. Linearization, discussed in the following paragraphs, is a means of using Ohm's law under a set of simplifying assumptions.

In a nonlinear system power is spread across the spectrum rather than being contained at the fundamental frequency. Applying Ohm's law to solve for circuit parameters assumes a linear relationship between voltage and current. To apply Ohm's law to a nonlinear system requires linearizing the system, and an understanding that solutions obtained are an approximation. Although electrode processes are inherently nonlinear, a linearization of the system can be done by considering only the voltage and current density at the fundamental frequency [46]. This linearization method is more accurate if the applied input voltage is kept small [46]. Unfortunately the voltage range given in [3] which is required to elicit the desired biological response dictates that the input voltage cannot be minimized. Therefore linearization should be considered a means for indicating general circuit behavior rather than for producing accurate absolute values.

By linearization and assuming $\mathrm{AC}$ behavior, the drive circuit of Fig. 5.3 can be described by:

$$
v_{s}=I\left(R_{i}+R_{o}+R m\right)
$$

where $v_{s}$ is the source voltage, $R_{i}$ is the interfacial polarization impedance, $R_{o}$ is the ohmic polarization impedance between the drive electrodes, $R_{m}$ is the current monitoring resistor, and $I$ is the effective current. 
In solving the circuit parameters for the electrolyte case, which is necessary to understand polarization effects, $R_{o}$ will be assumed to be linear, with an evaluation of this assumption provided further in this chapter. Since the recording electrodes have no significant electrode polarization effects, they provide a suitable measure of the resistivity of the electrolyte $\rho$, which can be used to determine $R_{o}$ :

$$
R_{o}=\rho \frac{d}{A}
$$

where $\rho$ is the resistivity of the electrolyte, $d$ is the length of the current path, and $A$ is the cross-sectional area of the current path [35]. Both $A$ and $\rho$ can be considered constant given the homogeneity of the electrolyte. To transform the resistance between the record electrodes, denoted by $R_{\text {rec }}$, to $R_{o}$ only the electrode distance $d$ needs to be considered. The drive electrodes are spaced $300 \mathrm{mil}(7.62 \mathrm{~mm})$ apart, while the recording electrodes are spaced $100 \mathrm{mil}(2.54 \mathrm{~mm})$ apart. Therefore,

$$
R_{o}=3 \cdot R_{r e c}
$$

To calculate $R_{\text {rec }}$, Ohm's law can be applied:

$$
R_{\text {rec }}=\frac{v_{\text {rec }}}{I}
$$

where $v_{r e c}$ is the voltage across the recording electrodes sampled by the DAQ and stored on the PC's nonvolatile memory, and $I$ is the effective current.

The effective current $I$ can be calculated using Ohm's law:

$$
I=\frac{v_{m}}{R_{m}}
$$

where $R_{m}$ is the nominal $470 \Omega$ current monitoring resistor, and $v_{m}$ denotes the voltage across $R_{m}$ as sampled by the DAQ and stored on the PC's nonvolatile memory. 
Combining (5.5), (5.6), and (5.7), $R_{o}$ can be written in terms of known parameters:

$$
R_{o}=3 \cdot R_{m}\left(\frac{v_{r e c}}{v_{m}}\right)
$$

Now that the $R_{o}$ contribution to electrode polarization can be calculated, the interfacial polarization impedance $R_{i}$ will be examined. $R_{i}$ can be linearized by considering the I$\mathrm{V}$ curve of Fig. 5.2 as piecewise linear. Given the $\mathrm{AC}$ voltage input, ideally $R_{i}$ is the inverse of the slope of the tangent to the point where on the I-V curve crosses $0 \mathrm{~V}$. For small voltages applied across the electrodes this tangent method will provide a reasonable approximation of $R_{i}$. Larger signals will have $R_{i}^{-1}$ approximated by a steeper sloped straight line. Therefore, as voltage or current in the electrode system increases, $R_{i}$ will tend to decrease. Rearranging (5.3) to solve for $R_{i}$ :

$$
\begin{aligned}
& R_{i}=\frac{v_{s}}{I}-R_{o}-R_{m} \\
& R_{i}=R_{m}\left(\frac{v_{s}}{v_{m}}-1\right)-R_{o} .
\end{aligned}
$$

With an understanding of $R_{i}$ and $R_{o}$, and the necessary equations to solve numerical values, the next task is to examine experimental data to understand how changes in the system can affect these values. This is important as the nonlinear artifacts generated by electrode polarization can mask the biological nonlinear response. Particular aspects that need to be examined are:

- How valid is the assumption of $R_{o}$ being linear?

- The addition of biological cells to the electrolyte may modify $R_{0}$. How will this affect the nonlinearity of $R_{i}$ ?

- The addition of glucose to the yeast suspension enables metabolic activity. How do metabolic byproducts affect the nonlinearity of $R_{i}$ ? 
The following analysis will answer these questions.

\subsection{Electrode Geometry Work}

\subsubsection{Evaluation of Ohmic Impedance}

To evaluate the linearity of $R_{o}$ and the effect of a changing $R_{o}$ on the system, an additional set of experiments were undertaken. As shown in (5.4), $R_{o}$ has a dependance on electrode spacing $d$. By examining the electrode-electrolyte system for two different electrode spacings, information can be obtained about the linearity of $R_{o}$ and about how changing $R_{o}$ can modify the system response.

In this experiment, data were collected in parallel for two different four-electrode configurations. One configuration was that used in the experimental work of Chapter 4 , in which each electrode was located $100 \mathrm{mil}(2.54 \mathrm{~mm})$ from the next. The second configuration had each electrode located $200 \mathrm{mil}(5.08 \mathrm{~mm})$ from the next, but was the same as the first configuration in all other aspects. Therefore the second configuration equaled the first scaled by two in terms of distance. Each of the electrode configurations was placed in a separate test container of electrolyte with a diameter of $30 \mathrm{~mm}$. A sinusoidal voltage $v_{s}$ of $1.5 \mathrm{~V}$ at $17 \mathrm{~Hz}$ was applied across the two parallel drive circuits, and data were collected for 30 seconds. The PSD results for the electrolyte response case are shown in Fig. 5.4.

The 100 mil spacing exhibited a fundamental with a magnitude of $-26 \mathrm{~dB}$ and a third harmonic $0.5 \mathrm{~dB}$ greater than the second. The 200 mil spacing exhibited a fundamental with a magnitude of $-21.8 \mathrm{~dB}$ and a second harmonic $4.5 \mathrm{~dB}$ greater than the third. Of particular interest here is the switch between a dominant second or a dominant third harmonic, solely by changing electrode spacing. It is also interesting that the 100 mil results do not exactly match those of the electrolyte case in 4.6.2. It is suspected that the increase in test container 

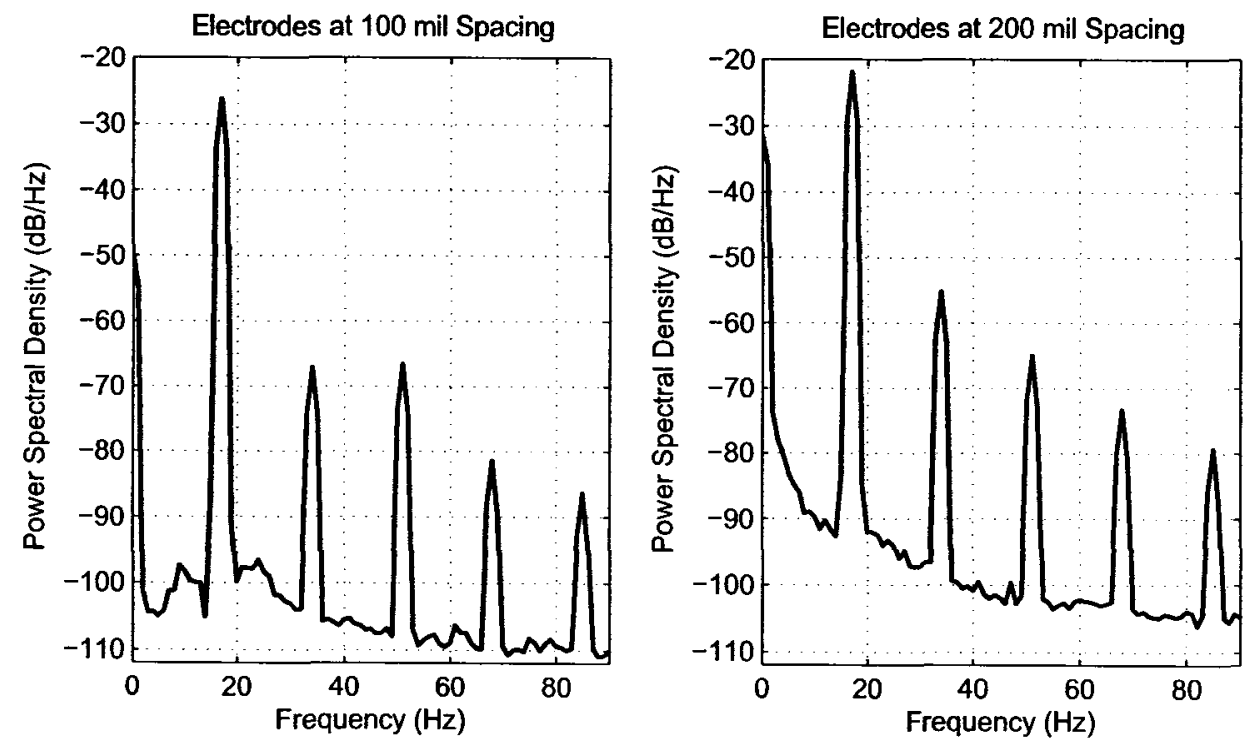

Figure 5.4: Effect of electrode spacing: The plot on the left shows the response PSD of the electrolyte solution with Ag-AgCl electrodes spaced at 100 mil on center. The plot on the right has electrodes spaced at 200 mil on center. This change in electrode geometry alters $R_{o}$, resulting in different harmonic content between the two configurations. 
size has altered the current density area, though further work is needed to confirm this.

The first aspect to examine is the change in $R_{\mathrm{o}}$ from the 100 mil configuration to the 200 mil configuration In this section a subscripted 1 denotes a parameter of the $100 \mathrm{mil}$ spacing, while a subscripted 2 denotes a parameter of the 200 mil spacing. To calculate $R_{o}$ using (5.8), values are required from the data. Voltages were determined by taking the average peak amplitude in the time domain over the 30 second time span. The values of $R_{m}$ were measured with a digital multimeter (Fluke 26III, Fluke Corporation, Everett, Washington). The values were found to be:

$$
\begin{aligned}
& v_{m 1}=651.3 \mathrm{mV} \\
& v_{m 2}=513.0 \mathrm{mV} \\
& v_{r e c 1}=82.42 \mathrm{mV} \\
& v_{r e c 2}=135.9 \mathrm{mV} \\
& R_{m 1}=472.3 \Omega \\
& R_{m 2}=469.1 \Omega .
\end{aligned}
$$

Using these values, and applying (5.8) yields:

$$
\begin{aligned}
& R_{o 1}=179.3 \Omega \\
& R_{o 2}=372.8 \Omega .
\end{aligned}
$$

If $R_{o}$ was strictly linear it would be expected that $2 \cdot R_{o 1}=R_{o 2}$, given the current path is twice the distance in the 200 mil configuration. The relative error due to nonlinearity of $R_{o}$ can be calculated as

$$
\begin{aligned}
& E r r=\frac{R_{o 2}-2 \cdot R_{o 1}}{R_{o 2}} \\
& E r r=3.8 \% .
\end{aligned}
$$


This $3.8 \%$ relative error due to nonlinearity is reasonably small and is consistent with what would be predicted. It can be concluded that treating $R_{o}$ as linear for this work is a reasonable simplifying assumption.

\subsubsection{Coupling of Interfacial and Ohmic Impedance}

With $R_{o}$ shown to be reasonably linear, the next objective is to examine how changes in $R_{o}$ affect $R_{i}$. Applying (5.9) and substituting in the values obtained previously, yields:

$$
\begin{aligned}
& R_{i 1}=436.1 \Omega \\
& R_{i 2}=529.7 \Omega .
\end{aligned}
$$

This increase in $R_{i}$ agrees with the analysis presented in Sec. 5.2.2 in which a decrease in current was shown to result in an increase in $R_{i}$. Increasing the electrode spacing from configuration 1 to configuration 2 results in $R_{o}$ increasing. The increase in $R_{o}$ causes the effective current $I$ to decrease. Applying (5.7) yields:

$$
\begin{aligned}
& I_{1}=1.386 \mathrm{~mA} \\
& I_{2}=1.088 \mathrm{~mA} .
\end{aligned}
$$

The decrease in $I$ results in $R_{i}^{-1}$ being approximated by a smaller piecewise linear portion of the I-V curve of Fig. 5.2, with a lower slope. Therefore $R_{i}$ increases as effective current decreases. It can be concluded that changes in $R_{o}$ modify the current through the system, resulting in a change in the nonlinearity of $R_{i}$. This difference in nonlinearity is observed in the different harmonic content of the two configurations shown in Fig. 5.4.

One additional aspect of Fig. 5.4 that requires explanation is the greater magnitude of the signal level in the 200 mil configuration, particularly at the fundamental frequency. 
Effective current decreases by $22 \%$ in configuration 2 . The impedance between the recording electrodes increases by $52 \%$ in configuration 2 . The increase in impedance dominates, resulting in the voltage measured across the recording electrodes being greater in configuration 2 . Therefore the voltage signal level across the recording electrodes for the $200 \mathrm{mil}$ configuration is greater than that of the 100 mil configuration.

\subsubsection{Effect of Cellular Bulk}

The final aspect to examine in this section is how the addition of yeast to the electrolyte affects $R_{o}$. In the previous section it was shown that $R_{o}$ could be modified by changing electrode spacing, resulting in different harmonic content occurring in the response signals. The addition of biological cells to the electrolyte can impede ionic conduction [35], resulting in $R_{o}$ being effectively increased.

To examine this effect, yeast at a concentration of $50 \mathrm{mg} \cdot \mathrm{mL}^{-1}$ was added to the electrolyte of the 100 mil electrode configuration. The suspension was allowed to stand for 20 minutes to allow the cells to rehydrate. After this rehydration period, the suspension was stirred to distribute the cells homogeneously, and data were recorded for 30 seconds. The plot of the quiescent yeast PSD for the 100 mil configuration is shown in Fig. 5.5.

Applying (5.8) with data obtained from the 100 mil configuration yielded a resistance between the drive electrodes $R_{o}$ of $179.4 \Omega$. This is essentially the same $R_{o}$ as was found in the configuration 1 electrolyte. It can be concluded that the addition of yeast does not change the resistance of the electrolyte at a perturbating frequency of $17 \mathrm{~Hz}$. At the low frequency employed, the cell membrane acts as an open circuit. Therefore there is no contribution to ionic conductivity of the intracellular fluid.

An interesting feature resulting from the addition of yeast is the increase in current 


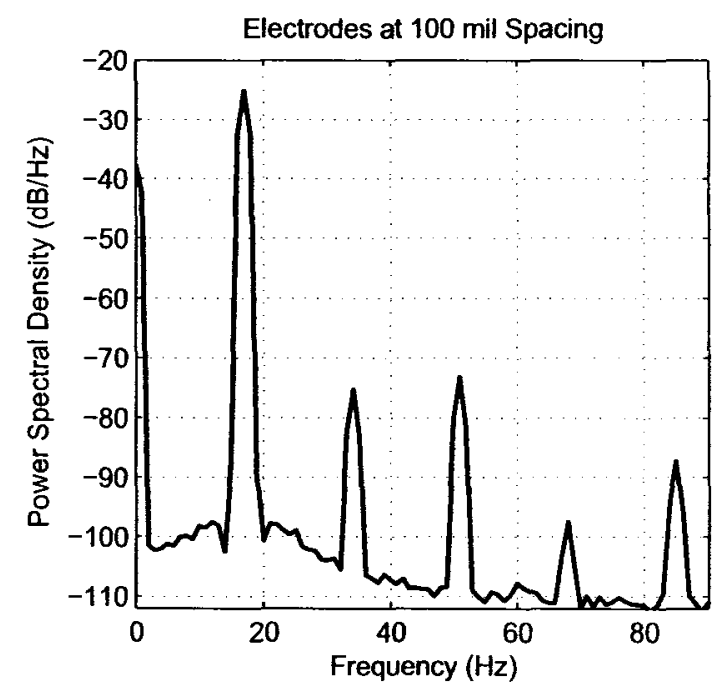

Figure 5.5: Quiescent yeast PSD: The plot of the quiescent yeast PSD when compared to the electrolyte data of Fig. 5.4 reveals that nonlinearity is reduced with the addition of yeast to the electrolyte.

which was observed. Effective current was found to be, using (5.7), $1.544 \mathrm{~mA}$. This implies that the addition of yeast modifies the interfacial impedance. Applying (5.9) and substituting in the values for the yeast in the 100 mil configuration gives $R_{i}=320.0 \Omega$. This is a $27 \%$ decrease in $R_{i}$ as compared to the electrolyte case.

The addition of yeast to the electrolyte causes a reduction in the interfacial polarization impedance $R_{i}$. Referring back to to the I-V curve of Fig. 5.2, it is apparent that a decrease in $R_{i}$ should be accompanied by a decrease in nonlinearity (see Sec. 5.2.1). Comparing the plots of the PSD of the electrolyte for the 100 mil configuration (see Fig. 5.4) with that of the quiescent yeast (see Fig. 5.5) the harmonic magnitudes are decreased in the quiescent yeast case, with little change in the fundamental. This provides strong evidence that the addition of yeast to the electrolyte modifies the interfacial polarization impedance. A reasonable hypothesis is that the cells physically disturb the the double layer of charge at the interface, reducing $C_{d}$ and $R_{d}$ in the electrode model of Fig. 5.1. Further work is 
required to understand the mechanism by which the biological cells modify $R_{i}$.

Reduced harmonics in the quiescent case as compared to the electrolyte are also found in the results of Fig. 4.25 and Fig. 4.21, comparing the points where the electrolyte fundamental has the same magnitude as the yeast fundamental. It is necessary to compare results at the same fundamental magnitude as minor differences in apparatus setup, such as electrode depth, can affect effective current.

\subsubsection{Summary}

To summarize, from this electrode geometry work two significant findings were made. The first of these is that the resistance of the electrolyte bulk $R_{o}$ is reasonably approximated as linear at the voltages and currents used in this work. The second important finding is that the addition of yeast cells to the electrolyte modifies the electrode interfacial polarization layer, reducing the interfacial polarization impedance $R_{i}$, and also reducing the harmonic content.

This analysis does not rule out the existence of biologically generated harmonics, but does indicate that nonlinear interfacial polarization impedance artifacts exist in the recorded data. With an understanding of how a suspension of quiescent yeast can generate nonlinear artifacts in the system, the next objective is to understand whether metabolically active yeast can also generate nonlinear artifacts.

\subsection{Current Variability}

The experimental data and accompanying analysis presented in Sec. 4.6.2 indicated that the linearized effective current varied over time in the three test cases. The greatest variation was observed in the active yeast case. This suggests that effective current might 
have a dependance on metabolic activity. Variation in effective current would modify the nonlinearity of the interfacial polarization impedance $R_{i}$ (see Sec. 5.2.1). An increase in effective current would cause the harmonic content in the response signal to increase, while a decrease in effective would current would cause the opposite. In attempting to identify the nonlinear response of the transmembrane protein, changes in harmonic content due to current variation could mask the biological signal of interest.

While the variation in magnitude at the fundamental frequency may be the result of current variation, another possibility is that the nonlinear behavior of the metabolic yeast is transferring power from the harmonics to the fundamental. To differentiate whether the variation in the fundamental is a system artifact or a biological response of interest it is necessary to examine the average power in the system over time. If in the active case average power is increasing over time, then metabolic activity is likely transducing energy from the glucose to increase the conductivity of the electrolyte. If in the active case average power remains constant, then some biological mechanism may be altering the harmonic content.

\subsubsection{Average Power Analysis}

The average power analysis can be performed in the frequency domain.

For a periodic waveform the PSD, denoted by $\mathscr{P}$, is given by

$$
\mathscr{P}=\sum_{n=-\infty}^{n=\infty}\left|c_{n}\right|^{2} \delta\left(f-n f_{0}\right),
$$

where the $c_{n}$ are the Fourier coefficients of the waveform, and $f_{0}$ is the fundamental frequency [47].

The $\left|c_{n}\right|^{2}$ terms can be be obtained numerically from the magnitude of the spectral components of the PSD plots. Given that the normalized average power $P_{\text {ave }}$ of the waveform 
is

$$
P_{\text {ave }}=\sum_{n=-\infty}^{n=\infty}\left|c_{n}\right|^{2}
$$

the magnitudes of the Fourier coefficients can be summed to evaluate the power change over time [47].

The following plot of Fig. 5.6 shows the normalized average power $P_{a v e}$ of the drive current signal for the three test cases of Sec. 4.6.2. To obtain this plot the magnitudes of the DC through fifth order spectral components were transformed from $\mathrm{dBs}$ to linear scale, summed and normalized, and then transformed back to $\mathrm{dBs}$.

This analysis reveals that average power increases the most in the active case, increases less in the quiescent case, and decreases slightly in the electrolyte medium. As average power is proportional to current, this plot also reflects the change in effective current of these cases. A hypothesis for the change in effective current can be found in Sec. 5.5.

The key finding of this section is that effective current is increasing significantly over time in the active yeast case. This increase will modify the nonlinearity of the interfacial polarization impedance $R_{i}$, resulting in artifacts occurring in the recorded signals. This also does not rule out that a nonlinear biological response is contained within the recorded data, but does indicate that some means of removing the artifact is necessary to observe this biological response. A method developed in this thesis to compensate for these artifacts is presented in Chapter 6. 


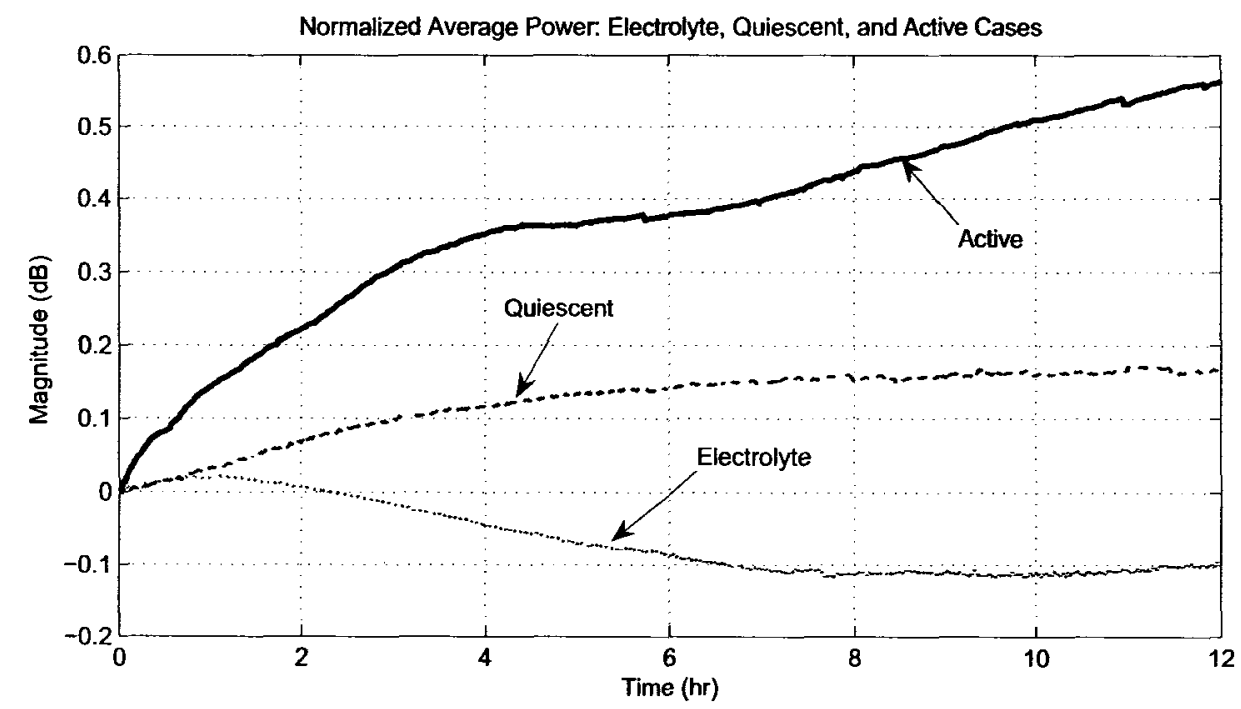

Figure 5.6: Time-course normalized average power: Drive current signals: The average power plots formed from drive current data indicate that current, and therefore system conductivity changes over time. The active yeast case shows the greatest increase in conductivity, while the quiescent case shows a smaller increases. The electrolyte is characterized by a slight decrease in conductivity.

\subsection{Metabolic Byproducts}

\subsubsection{Conductivity and $\mathrm{CO}_{2}$}

The average power analysis showed that conductivity in the active yeast increased significantly over time. There are a number of factors that can affect conductivity in the system. Conductivity is directly proportional to the surface area of the electrodes, and is inversely proportional to both the electrode spacing and the thickness of the chloride layer [35, 37]. Conductivity is also directly proportional to ion concentration, ion mobility, and temperature $[35,37]$. These test cases were all run in parallel so changes in temperature and electrode properties exhibit the same approximate change in conductivity. The factor that is likely to change only under metabolically active conditions is ion concentration.

$S$. cerevisiae is a facultatively aerobic organism. Depending on environmental condi- 
tions, primarily concentration of glucose and oxygen, metabolism can occur through either aerobic oxidation or anaerobic fermentation, with both reactions producing $\mathrm{CO}_{2}$ gas. Aerobic respiration is the complete breakdown of glucose through oxidation:

$\mathrm{C}_{6} \mathrm{H}_{12} \mathrm{O}_{6}+6 \mathrm{O}_{2} \rightarrow 6 \mathrm{CO}_{2}+6 \mathrm{H}_{2} \mathrm{O}+38$ ATP molecules $\quad[48]$

Anaerobic fermentation requires no oxygen:

$\mathrm{C}_{6} \mathrm{H}_{12} \mathrm{O}_{6} \rightarrow 2 \mathrm{CO}_{2}+2 \mathrm{C}_{2} \mathrm{H}_{5} \mathrm{OH}+2$ ATP molecules [48]

An initial glucose concentration of $170 \mathrm{mmol} \cdot \mathrm{L}^{-1}$ was supplied and a final concentration after 12 hours was measured at $25 \mathrm{mmol} \cdot \mathrm{L}^{-1}$. In this experiment approximately 0.3 mmol of glucose was consumed over 12 hours. Given the high glucose concentration and available oxygen it is reasonable to assume that metabolic activity is primarily aerobic. Assuming aerobic respiration, the consumed glucose produces $1.8 \mathrm{mmol}$ of $\mathrm{CO}_{2}$ gas. From the ideal-gas law, $P V=n R T$, approximately $44 \mathrm{~mL}$ of $\mathrm{CO}_{2}$ gas is bubbled through the suspension. A portion of this $\mathrm{CO}_{2}$ will dissolve yielding the reaction :

$\mathrm{CO}_{2}(\mathrm{aq})+\mathrm{H}_{2} \mathrm{O} \rightleftarrows \mathrm{H}_{2} \mathrm{CO}_{3}(\mathrm{aq}) \rightleftarrows \mathrm{H}^{+}(\mathrm{aq})+\mathrm{HCO}_{3}^{-}(\mathrm{aq}) \quad$ [48].

This results in an increase in highly mobile $\mathrm{H}^{+}$ions, and a corresponding increase in conductivity. An electrode-based sensor to measure the conductivity change caused by dissolving $\mathrm{CO}_{2}$ in water leverages this effect [49].

\subsubsection{Bubble Production and $\mathrm{CO}_{2}$}

The production of gaseous $\mathrm{CO}_{2}$ as a metabolic byproduct results in bubbles perfusing through the suspension. The physical effect of the bubbles on the dielectric properties of the active yeast suspension was considered as a factor that could give rise to an artifact [50].

To see if the bubbles themselves were causing a change in the harmonics of the active 
case, air was bubbled through a resting yeast suspension. A small pump was used to force air through a diffuser placed at the bottom of a suspension of quiescent yeast. This caused the air to form fine bubbles which rose to the surface. As Fig. 5.7 shows, this resulted in the noise floor of the spectrum rising significantly, but did not cause a change in the harmonic signature.
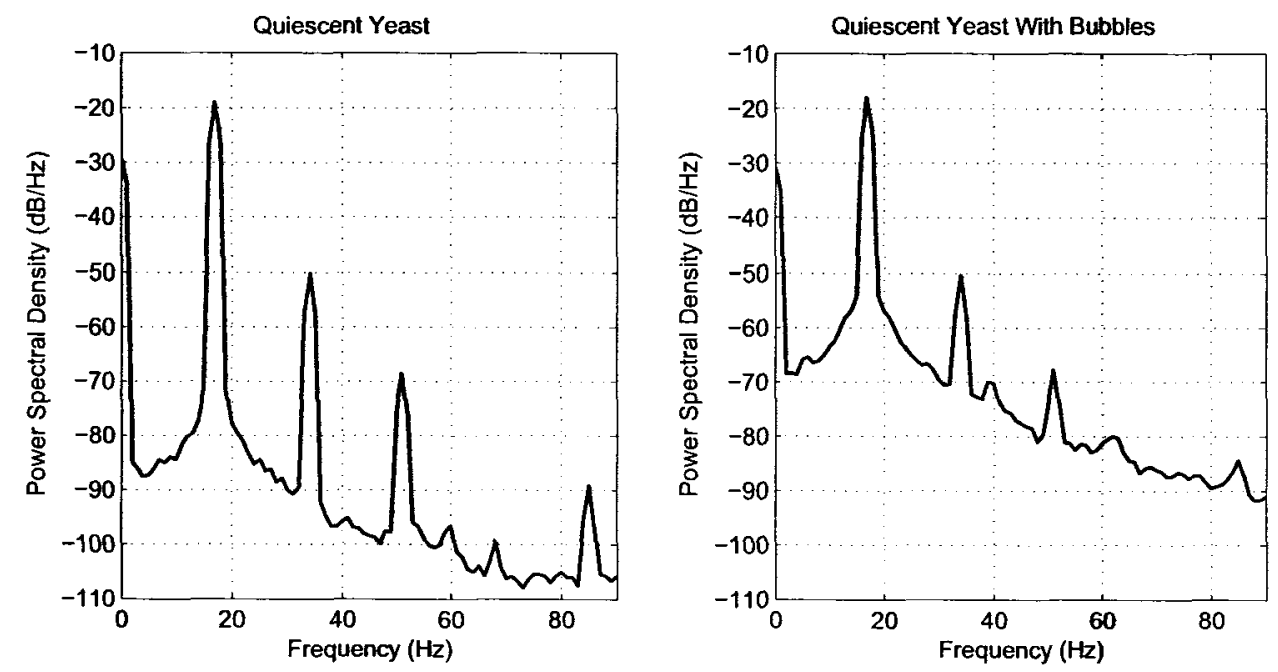

Figure 5.7: Effect of air bubbles: Air was bubbled through a suspension of quiescent yeast. This raised the noise floor but did not affect the harmonic magnitudes.

This demonstrates that the physical effect of the $\mathrm{CO}_{2}$ gas bubbles, produced through metabolic activity, does not alter the harmonic magnitudes.

\subsection{Summary}

A number of key findings were made in this analysis chapter. The first of these was that the ohmic polarization impedance due to the electrolyte bulk is well approximated as linear at the voltage and current range employed in the experimental work. The second important finding is that the addition of yeast cells to the electrolyte modifies the electrode interfacial 
polarization layer, reducing the interfacial polarization impedance $R_{i}$, and also reducing the harmonic content. From this it can be concluded that the addition of yeast to the electrolyte induces a nonlinear polarization artifact.

The next important finding was that metabolic byproducts also introduce an electrode polarization artifact in the system, independent of any transmembrane response. Effective current was found to increase significantly in the active yeast case, with the hypothesis that metabolically-produced $\mathrm{CO}_{2}$ gas was reacting to produce highly conductive hydrogen ions.

Finally, the physical effect of air bubbles in a suspension of quiescent yeast was examined. This was done to simulate the physical effect of $\mathrm{CO}_{2}$ gas bubbles in the metabolically active case. While the bubbles caused the noise floor to increase, no significant effect on the harmonic response was found.

In conclusion, the analysis work of this chapter found that electrode polarization effects are the primary mechanism in defining the harmonic response. These polarization effects can serve to mask the nonlinear response of the $\mathrm{H}^{+}$ATPase protein. Therefore methods are needed to compensate for the nonlinear artifacts of electrode polarization. The following chapter will present methods to compensate for these artifacts. 


\section{Chapter 6}

\section{Artifact Compensation Methods}

\subsection{Overview}

The analysis presented in Chapter 5 revealed that nonlinear artifacts occur in the system which can mask the nonlinear biological response of interest. While these artifacts can be induced by different elements within the system, the ultimate source of the nonlinear artifacts is electrode polarization. The interfacial polarization determines the initial state of the nonlinearity in the electrolyte solution. Changes to the system which modify the current density, such as the addition of biological cells, or an increase in conductivity through metabolic byproducts, cause a deviation in the nonlinearity from the initial state.

In the metabolically active yeast, conductivity was found to increase, resulting in variation of the nonlinear interfacial polarization. To observe the nonlinear response of the $\mathrm{H}^{+}$ATPase protein under metabolically active conditions, a means of compensating for the variation in current which induces the interfacial polarization artifact is required.

The following chapter first presents an empirical model to describe the input-output relationship of the system under test. This model is then applied to two novel compensation techniques. The first technique compensates for current variation, while the second compensates for electrode polarization artifacts. 


\subsection{System Model}

Schwan, in his work on electrode polarization, employed a Taylor series expansion as an empirical model to describe admittance at the polarized interface [51]. Moussavi et al., following up on Schwan's model, developed this Taylor series model further [36]. More recent work has seen a theoretical model for electrode polarization based on the Butler-Volmer equation, which takes into account the underlying electrochemical processes occurring at the electrode interface [52].

Based on the success of the Taylor series to model admittance at the electrode interface in [51] and [36], a Taylor series expansion was employed in this work to empirically model the voltage output-input relationship in the electrode-electrolyte system and in the yeastelectrode-electrolyte system.

If the attenuation in the system was linear, the output-input relationship could be modelled as:

$$
v_{o}\left(v_{i n}(t)\right)=k v_{i n}(t)
$$

where $k$ represents the attenuation factor, $v_{o}(t)$ is the output voltage, and $v_{i n}(t)$ is the input voltage.

As the analysis in Chapter 5 revealed, the signal attenuation due to the interfacial polarization is nonlinear. To model this nonlinear attenuation, a Taylor series expansion of $v_{0}\left(v_{i n}(t)\right)$ about an input $v_{i n}(t)=0$ is formed:

$$
\begin{aligned}
v_{o}\left(v_{i n}(t)\right) & =k_{0}+k_{1} v_{i n}(t)+k_{2} v_{i n}^{2}(t)+k_{3} v_{i n}^{3}+\ldots \\
& =\sum_{n=0}^{\infty} k_{n} v_{i n}^{n}(t)
\end{aligned}
$$


Applying a sinusoidal input $v_{i n}(t)=A \sin \left(2 \pi F_{i n} t\right)$ to (6.2), expanding, and employing trigonometric identities, will yield the parametric $k_{n}$ contribution, and input amplitude $A$ contribution to the spectral components. This is illustrated for the first four non-DC terms, which can be easily determined analytically:

$$
\begin{aligned}
& k_{1} v_{i n}(t)=k_{1}\left[A \sin \left(2 \pi F_{i n} t\right)\right] \\
& k_{2} v_{i n}^{2}(t)=k_{2}\left[\frac{A^{2}}{2}-\frac{A^{2}}{2} \cos \left(2 \cdot 2 \pi F_{i n} t\right)\right] \\
& k_{3} v_{i n}^{3}(t)=k_{3}\left[\frac{A^{3}}{2} \sin \left(2 \pi F_{i n} t\right)-\frac{A^{3}}{4} \sin \left(3 \cdot 2 \pi F_{i n} t\right)\right] \\
& k_{4} v_{i n}^{4}(t)=k_{4}\left[\frac{A^{4}}{4}+\frac{A^{4}}{8}+\frac{A^{4}}{2} \cos \left(2 \cdot 2 \pi F_{i n} t\right)-\frac{A^{4}}{8} \cos \left(4 \cdot 2 \pi F_{i n} t\right)\right]
\end{aligned}
$$

This expansion illustrates how both input amplitude $A$ and the $k_{n}$ coefficients contribute to the spectral components. To compensate for changes in the system over time, the methods presented in the following sections will use the model of (6.2).

\subsection{A Compensation Method for Variation in Current}

The analysis of current variability presented in Section 5.4 showed that the effective current supplied to the system varies with time. The active case in particular showed a significant increase in effective current over time. As was also discussed in the analysis, the interfacial impedance $R_{i}$ is nonlinear, and tends to decrease as current increases. Therefore variation in effective current will modify the harmonic content observed in the system, which can mask the nonlinear biological signal of interest. Providing a means to compensate for the variation in effective current could help to reveal the biological signal. 
Varying inversely with effective current in this apparatus are the interfacial impedance $R_{i}$, and the voltage across the drive electrodes $v_{e f f}$. To compensate for variation in the effective current, the Taylor series expansion given in (6.2) is employed as a transfer function to model the system input-output relationship at a time point. In (6.2), the $k_{n}$ coefficients represent the transfer function of this system. This transfer function includes attenuation due to $Z_{i}$ at an effective current, as well as any biological response. The $v_{i n}$ term represents a linearly scaled $v_{e f f}$, the perturbating sinusoidal voltage. Because the ohmic impedance $R_{o}$ is in the linear range and the recording electrodes are transparent (see Sec. 5.3), the amplitude of $v_{i n}$ can be set to the peak voltage at the recording electrodes.

Since $v_{o}$ and $v_{\text {in }}$ are known, numerical methods can be used to find the transfer function. This process can be applied to each time point, thus obtaining a representation of the system over time.

Knowledge of the transfer function over time allows simulating an output for a new input. In particular, to compensate for the amplitude variation in $v_{\text {in }}$ caused by the effective current variation, the system can be simulated for a constant amplitude $v_{i n}$. Unfortunately the $k_{n}$ coefficients representing $R_{i}$ are a function of effective current. Therefore the compensation method cannot account for the changes in interfacial polarization in simulating a constant effective current. The compensation output will be least accurate where current change is the greatest.

Despite the problem this method has in accounting for the fact that interfacial polarization is a function of effective current, it is still worth pursuing as a first attempt in minimizing system artifacts.

To apply the current variation compensation method, equation (6.2) was truncated after 
the seventh order term:

$$
\begin{aligned}
v_{o} & =k_{0}+k_{1} v_{i n}+k_{2} v_{i n}^{2}+k_{3} v_{i n}^{3}+\cdots+k_{7} v_{i n}^{7} \\
& =\sum_{n=0}^{7} k_{n} v_{i n}^{n} .
\end{aligned}
$$

The truncation choice was determined empirically by increasing the order of the Taylor series until the output was stable. The model was then fit using a nonlinear least squares method to the experimental data sampled at the recording electrodes to obtain the $k_{n}$ coefficients at each six second time point. Thus a time-course of coefficients describing the system at an input voltage $v_{i n}$ was obtained.

In the curve fitting process, at each time point the amplitude $A$ was determined in the time domain by measuring the peak to peak voltage of the yeast response signal. This linear approximation of $A$ was then used in the model to describe the voltage $v_{i n}$ across the electrolyte response electrodes:

$$
v_{i n}=A \sin \left(2 \pi F_{i n} t\right)
$$

Equation (6.3), with the time-course of $k_{n}$ coefficients describing the experimental data, was then solved numerically for $v_{i n}=B \sin \left(2 \pi F_{i n} t\right)$ to simulate the effect of constant amplitude $B$ on harmonic behavior. To minimize the distortion of any biological response, the value of $B$ was calculated as the average $v_{i n}$ over the total time.

Additional processing of the simulation output was performed to smooth the compensation output plots. The simulation output were first filtered on quality of fit data returned by the curve fitting algorithm. This enabled discarding points below a goodness threshold. The second filter employed was an 80 point ( 8 minute) moving average. This filter was used to reduce the effect of the curve fitting algorithm tending to converge in either the positive or negative direction depending on the initial guess. This convergence phenomenon was 
due to the optimization method used to reduce the curve fit processing time. In this optimization method the last $k_{n}$ values solved are used as the next initial guess. This can cause the output to systematically drift in one direction. To minimize this drift, a new random initial guess was made for each five minutes of data processed. Additionally, when more than 800 iterations were required, the initial guess was flagged as bad and a new random initial guess made.

\subsubsection{Current Variation Compensation Results}

The results of the simulation to compensate for current variation in the electrolyte, quiescent, and active cases are shown in Fig. 6.1.

The compensated electrolyte case is shown in the top plot of Fig. 6.1. Because conductivity variation in the electrolyte is small, it was expected that the compensation output would resemble the results of the uncompensated data (see Fig. 4.25). This is the case for the relative magnitudes of the second and third harmonic magnitudes. Both the compensated and the uncompensated data are characterized by a dominant second harmonic. Differing between these two outputs is approximately a $-10 \mathrm{~dB}$ shift in the absolute magnitude of the second and third harmonics in the compensated output. The compensated data also exhibits some noise resulting from the fitting process.

The compensated quiescent case, shown in the middle plot of Fig. 6.1, also closely resembles the corresponding uncompensated data (see Fig. 4.25). This was expected as the conductivity decrease being compensated for in the quiescent case is small. Similar between these two outputs is the relative magnitude of the second and third harmonics. The absolute magnitude differs though, with a $-10 \mathrm{~dB}$ shift in the compensated output. The compensated data also exhibits some noise resulting from the fitting process. 

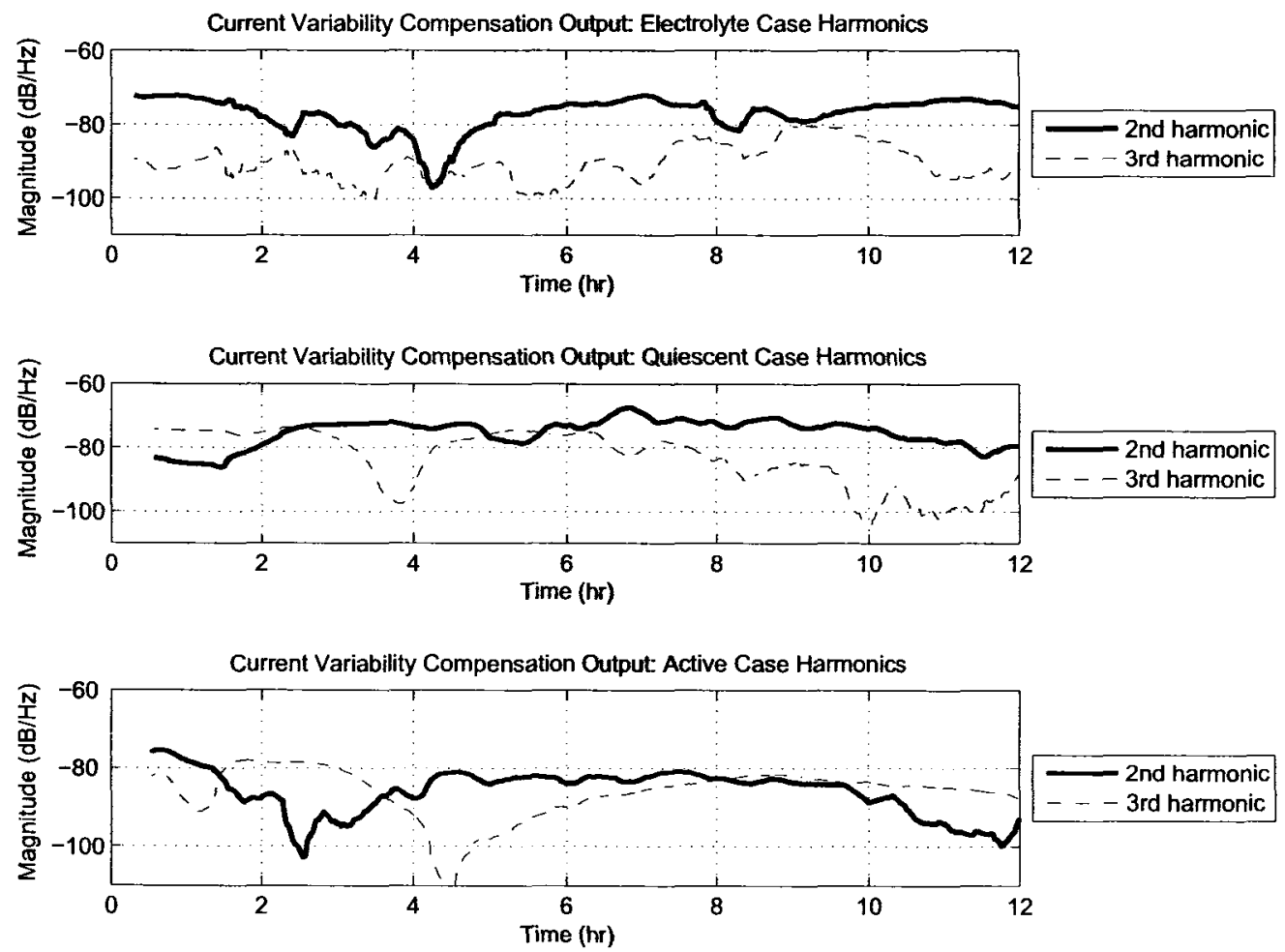

Figure 6.1: Current compensation method output: Electrolyte, quiescent yeast and active yeast: Using the described method to compensate for variation in effective current, the system response can be simulated for a constant input voltage. The plots show the magnitude of the second and third harmonics for the electrolyte, the quiescent yeast, and the active yeast cases respectively. 
The active case exhibited the largest variation in current over time, and as such was expected to display a different harmonic signature than that of the uncompensated active case. This expectation was met in comparing the compensated and uncompensated outputs. The compensated active case, shown in the bottom plot of Fig. 6.1, is characterized by approximately four phases of relative harmonic magnitude. Phase one (hours 0-1.5) and phase three (hours 4-8) show a second harmonic greater than the third. Phases two (hours 1.5-4) and four (hours 8-12) show a third harmonic greater than the second. These results appear quite different from the uncompensated active data which are characterized by only three phases (see Fig. 4.25). The dominant harmonic is also reversed as compared to the uncompensated results up until hour eight.

Unfortunately, the compensation method adds noise to the results. This noise results from error in the curve fit, which could be reduced by lowering the curve fit tolerance, but with the tradeoff of increased processing time. Instead, to reduce noise poor, quality fits were discarded, and the data were low-pass filtered. Another problem with this method is a reduction in the absolute magnitude of the second and third harmonics. Further analysis is required to understand this phenomenon.

As discussed earlier, the current compensation method is unable to account for the changes in interfacial impedance, which is a function of effective current. The results therefore still contain electrode polarization artifacts. The active case in particular displays the strongest artifacts as it has greatest current change. The artifacts in the compensation output do not represent the harmonic content of the the interfacial impedance accurately because impedance is a function of effective current. The large difference between the harmonic magnitudes of the compensated active case and the uncompensated active case are likely the result of this current dependance problem. Therefore additional work needs 
to be undertaken to compensate for polarization artifacts before the true biological response can be revealed. In the following section this compensation method is developed further in an attempt to reduce electrode polarization effects.

\subsection{A Method to Compensate for Electrode Polarization}

The analysis of the electrode-electrolyte system presented in Section 5.3 showed that interfacial electrode polarization impedance introduces a nonlinear artifact into the system. This artifact is caused by the current injecting electrodes, while the recording electrodes can be considered transparent. Because the artifact-inducing polarization impedance appears in series with the biological cells, separating the nonlinearity of the cells from the electrode artifacts is difficult.

One means of separating the polarization impedance from the biological impedance is to consider two distinct data sets. One data set, that of the electrolyte case, contains only the nonlinear interfacial impedance $R_{i}$ and the linear impedance $R_{o}$. This first data set can be considered as the polarization impedance reference. The other data set represents a suspension of the biological cells in the same electrolyte solution. In this second data set, the contribution of the nonlinear polarization impedance $R_{i}$ is included, and possibly a biologically modified $R_{o}$.

The theoretical development of a nonlinear response due to the transmembrane protein $\mathrm{H}^{+}$ATPase assumes a sinusoidal perturbating source [27]. In practice, the nonlinear interfacial electrode polarization impedance results in a nonlinear perturbating voltage and current density stimulating the biological sample. Therefore electrode polarization artifacts elicit a biological response from a nonlinear stimulus input. As such, it is not enough to 
isolate the biological response from the polarization impedance. A means of compensating for the nonlinear stimulus applied to the cells is necessary to reveal the biological response to a pure sinusoidal stimulus. The compensation method presented in this section simulates the biological response to a stimulus containing no polarization artifacts.

The approach used to compensate for electrode polarization artifacts is similar to that used in compensating for variation in current (see Section 6.3), in that the model given by (6.2) is fit to the data. However, this polarization compensation method fuses data obtained in parallel from both the electrolyte case and the biological case of interest. The assumption made in fusing the data is that the addition of yeast to the electrolyte does not modify the polarization layer, so that the same polarization effects are found in both the electrolyte and biological data sets. Given this assumption, the electrolyte response signal can be considered as the perturbating input to the yeast sample at the record electrodes. The biological signal then is in response to the nonlinear input of the polarization artifact. Knowing the polarization artifact (from the electrolyte data), and knowing the biological response (from the yeast suspension data) to this artifact enables simulating a biological response to an artifact-free input.

The first aspect of the polarization compensation method is to form a model describing the interfacial polarization artifact as observed at the recording electrodes. For a pure sinusoid applied across the drive electrodes, the interfacial impedance results in a nonlinear attenuation of the input voltage. The applied sinusoidal voltage $v_{i n}$ is given by:

$$
v_{i n}(t)=A \sin \left(2 \pi F_{i n} t\right)
$$

As the analysis of Sec. 5.3 indicated, the resistance of the electrolyte $R_{o}$ is linear, and the recording electrodes are transparent. Therefore, to transform the voltage applied across the drive electrodes to that which would be observed at the recording electrodes, $A$ is a 
linear term representing an attenuated input amplitude. In implementing the compensation method, a numerical value of $A$ was determined by measuring the peak AC amplitude of the voltage waveform at the response electrodes.

To model the output resulting from the nonlinear attenuation of the input voltage, the interfacial polarization impedance model of (6.2) was used. Equation (6.2) was truncated after the seventh order term to give:

$$
\begin{aligned}
v_{o} & =k_{0}+k_{1} v_{i n}+k_{2} v_{i n}^{2}+k_{3} v_{i n}^{3}+\cdots+k_{7} v_{i n}^{7} \\
& =\sum_{n=0}^{7} k_{n} v_{i n}^{n} . \\
& \equiv v_{p}
\end{aligned}
$$

The seventh order truncation was chosen empirically as the minimum order in which the first five harmonics were stable. The model was then fit using a nonlinear least squares method to the electrolyte data sampled at the recording electrodes. This fitting process was repeated for each data record occurring every six seconds over the twelve hour total test time. From this method, a time course of $k_{n}$ coefficients were obtained to describe the effect of interfacial polarization impedance as observed in the voltage signal of the recording electrodes.

With the coefficients representing the effect of interfacial impedance as observed at the record electrodes determined, the next part of the compensation method is to consider $v_{p}$ as the perturbating input to the biological sample. To implement this aspect, a Taylor series expansion was chosen to model the biological effect on $R_{o}$. Because biologically produced harmonics are expected, the choice of this model can be expected to reflect the system behavior accurately.

Equation (6.2) was truncated after the seventh order term and fit using a nonlinear least squares method to the yeast data sampled at the record electrodes to obtain the $m_{n}$ 
parameters:

$$
\begin{aligned}
v_{\boldsymbol{o}} & =m_{0}+m_{1} v_{p}+m_{2} v_{p}^{2}+m_{3} v_{p}^{3}+\cdots+m_{7} v_{p}^{7} \\
& =\sum_{n=0}^{7} m_{n} v_{p}^{n} .
\end{aligned}
$$

The $m_{n}$ parameters model the biological response to the polarization dependant nonlinear perturbating electric field.

To examine the biological response to an artifact-free input, the biological response to a pure sinusoid was simulated by using these $m_{n}$ parameters and letting

$$
v_{p}(t)=A \sin \left(2 \pi F_{i n} t\right)
$$

The resulting output, shown in Figure 6.2, gives the biological response without the nonlinear polarization artifacts.

Additional processing of the simulation output was performed to smooth the compensation output plots. The simulation output were first filtered on quality of fit data returned by the curve fitting algorithm. This enabled discarding points below a goodness threshold. The second filter employed was an 120 point (12 minute) moving average. This filter was used to reduce the effect of the curve fitting algorithm tending to converge in either the positive or negative direction depending on the initial guess. This convergence phenomenon was due to the optimization method used to reduce the curve fit processing time. In this optimization method the last $k_{n} / m_{n}$ values solved are used as the next initial guess. This can cause the output to systematically drift in one direction. To minimize this drift, a new random initial guess was made for each five minutes of data processed. Additionally, when more than 800 iterations were required, the initial guess was flagged as bad and a new random initial guess made. 


\subsubsection{Polarization Compensation Results}

The plots for both the quiescent and active case compensation results show a strong similarity between the second and third harmonic behavior over time. The compensated active and quiescent second harmonic patterns have a correlation coefficient of 0.84 . The compensated active and quiescent third harmonic patterns have a correlation coefficient of 0.60 . From hours 0 to 1 and hours 3 to 12, both the active and quiescent cases exhibit a similar pattern in terms of second and third harmonic magnitude. Between hours 1 and 3, the two cases display a different pattern. During this period in the quiescent case, the third harmonic is greater than the second. In this same time period for the active case, the second harmonic magnitude is greater than or equal to that of the third. The period of greatest metabolic activity in the active case occurs between hours 1 and 5 . Therefore in the first half of the highly metabolic period, a different harmonic pattern occurs in the two cases.

Before any conclusions can be drawn from the electrode polarization compensation results, the assumption made in implementing this method must be examined. It was assumed that the addition of yeast cells to the electrolyte did not alter the interfacial polarization impedance $R_{i}$. The analysis work of Sec. 5.3 indicated that the addition of yeast to the electrolyte reduced the interfacial polarization impedance $R_{i}$. This compensation method does not account for any yeast-induced polarization effects. Therefore, yeast-induced polarization effects can occur in the compensation output. Returning to the difference between the two cases observed from hours 1 to 3, two scenarios causing this difference can be considered. The first scenario is that the cell transmembrane protein, as described in [3], is imparting a nonlinear effect on the system. The second scenario is that the yeast cells are modifying $R_{i}$ differently during this highly active period.

In considering the first scenario, that the $\mathrm{H}^{+}$ATPase protein is imparting a nonlinear 

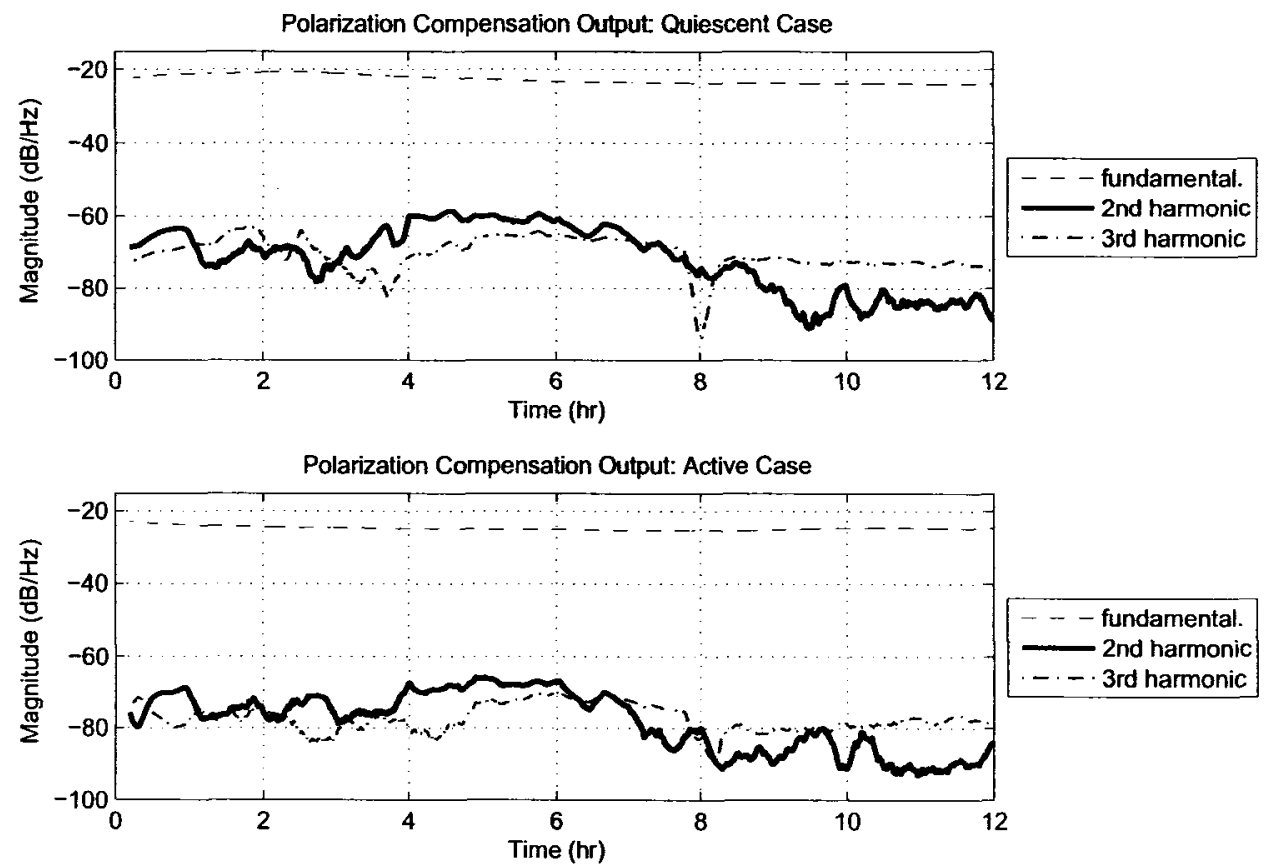

Figure 6.2: Polarization compensation method output: Electrolyte, quiescent yeast and active yeast: Using the described method to compensate for polarization artifacts, the system response can be simulated for a linear perturbating input. The top plot shows the magnitude of the fundamental, and second and third harmonics for the quiescent yeast, while the bottom plot displays those of the active yeast case. 
response onto the system, there is no evidence to confirm or deny this. Becuase the yeast can modify the interfacial polarization layer, and this compensation method does not remove this effect, any protein response could still be masked. In considering the second scenario, that the active yeast can modify the interfacial polarization layer differently than the quiescent yeast, more evidence supporting this can be found. The hypothesis presented in Sec. 5.3 for the change in interfacial impedance through the addition of yeast surmised that the yeast cellular bulk disturbed the double layer of charge at the electrode-electrolyte interface, resulting in a change in $R_{i}$.

The yeast species used in this work, S.cereviaiae, is a top-fermenting yeast. While metabolizing glucose, the cells move towards the surface of the test container. After reproducing, the yeast cells flocculate and drop to the bottom of the container. Through flocculation, several yeast cells link together. It is reasonable to expect that the movement of yeast cells from bottom to top and back, would cause some dynamic behavior at the electrode-electrolyte interface. The hypothesis then, is that during the first half of the highly active period, the movement of single yeast cells towards the surface disturbs and alters the interfacial double layer of charge. This causes the change in the harmonic pattern to differ between the active and quiescent cases during hours 1 to 3 . After this time the metabolically active yeast will tend to clump together and settle. The movement of these cell clumps do not have the same effect as single cells on the polarization layer.

From hours 5 to 12 the active and quiescent cases display similar harmonic patterns. As cell movement is considerably reduced during this period in the active case and is negligible in the quiescent case, this observation adds further support to the hypothesis that cell movement affects the polarization layer. Based on the evidence that cellular bulk can modify $R_{i}$, and that cellular bulk undergoes dynamic movement while metabolically active, the 
argument can be made that electrode polarization effects dominate the recorded data rather than a transmembrane protein effect.

\subsection{Summary}

This chapter discussed two methods developed to compensate for system artifacts which can serve to mask the biological response in NLDS of yeast cells. In these compensation methods an empirical model using a Taylor series expansion was formed to describe the effect of a nonlinear impedance on an input voltage. This model was then fit to the timecourse data to obtain a numeric representation of the signal of interest. This model served as the basis for the two compensation methods.

The first method compensated for a variation in effective current over time. An earlier analysis found that current in the active case increases somewhat over time, while the quiescent and electrolyte cases show a smaller increase and decrease respectively. This variation in effective current modifies the nonlinearity of the polarization impedance, resulting in changes in the harmonic magnitudes which can mask the biological harmonics of interest. By inputting a constant amplitude sinusoid to the system model, the response to a constant effective current over time was simulated. This method requires some improvement to account for the fact that interfacial polarization impedance is a function of effective current.

The second method compensated for electrode polarization effects by employing the electrolyte solution as a polarization reference. Electrode polarization effects transform the effective stimulus current from linear to nonlinear, resulting in a nonlinear stimulus to the yeast samples. This nonlinear stimulus can serve to mask the biological response. To compensate for polarization artifacts the empirical model was first fit to the electrolyte data 
to obtain the polarization spectrum parameters. The model was then fit to the yeast case data, using the polarization spectrum parameters as the input. The resulting model was then used to simulate the biological response to a sinusoidal input, to yield the system response in the absence of polarization artifacts. The result of this compensation method revealed little difference between the harmonic pattern of the active case and that of the quiescent case. This provides further evidence that interfacial polarization impedance is modified by the local presence of cellular bulk. 


\section{Chapter 7}

\section{Discussion}

\subsection{Overview}

The following chapter synthesizes the knowledge gained through the work of this thesis in the form of a discussion. The first section briefly summarizes the experimental results and the findings of the results analysis. The second section discusses the effect of cellular bulk on interfacial polarization artifacts. The following third section then discusses the compensation methods developed in this thesis. The fourth section presents some practical aspects of implementing NLDS in blood glucose determination. The fifth section discusses directions for future work.

\subsection{Thesis Work}

The work of this thesis, undertaken with the goal of evaluating NLDS as a means of predicting glucose concentrations, found that electrode polarization artifacts are a significant problem. The hypothesis was that through experimentation, a transmembrane protein nonlinear response correlated with metabolic activity in a suspension of yeast cells would be observed. The theory describing this phenomena was presented in [27], and the experimen- 
tal work of [3] indicated a transmembrane protein effect could be observed.

Initial experimentation focussed on repeating some of the experimental work of [3]. This set of experiments, in which gold electrodes were used, found significant nonlinearities in the recorded data, and the results of [3] could not consistently be repeated. To reduce the nonlinear artifacts, modifications were made to the experimental apparatus and protocol. The gold electrodes were replaced with $\mathrm{Ag}-\mathrm{AgCl}$ to reduce the magnitude of the nonlinear electrode effects. Three cases were examined in detail, and several trials were conducted using this new test protocol. The three cases were the electrolyte solution, a suspension of quiescent yeast, and a suspension of yeast actively metabolizing glucose. These experiments found that the harmonic content in the electrolyte and quiescent yeast were fairly stable as compared to the active case. The difference in stability between the active case and the other two cases gave the appearance of some nonlinear behavior due to metabolic activity in the active case. Further analysis of the active case found that the effective current increased significantly in this case, whereas there were only small changes in effective current in the quiescent and electrolyte cases. This discovery led to the hypothesis that metabolic byproducts were increasing the concentration of ions in the active case, resulting in a change in effective current.

In the relevant literature, electrode polarization impedance is known to be nonlinear, in particular at high current densities. Since electrode polarization impedance can be a function of current, variation in effective current can modify the harmonic content. Due to this, it was necessary to understand the artifacts created by electrode polarization before attempting to make conclusions regarding a biological response. The analysis of electrode polarization found that the interfacial polarization impedance was in the nonlinear range, and also that it was altered by the addition of cellular bulk to the electrolyte. Research 
by others, discussed in the following section, provides further evidence that the interfacial impedance can be altered by the addition of cells.

\subsection{Effect of Cellular Bulk}

It has been suggested that the harmonic signature attributed to transmembrane protein effects is a result of the addition of yeast, which modifies the system nonlinearities in a repeatable fashion [7]. Since the work of [7] was performed using a constant current source stimulus, and the experiment was conducted using a different test protocol than [3], a direct comparison between their respective results can not be made. In this thesis work an apparatus and test protocol more closely aligned with that of [3] were used. The results obtained provide strong evidence that the addition of cells to an electrolyte solution modifies the interfacial polarization.

Further evidence of the effect of cellular bulk on electrode interfacial polarization is found in the work of others on colloidal suspensions. The early low frequency work with colloids, in particular with suspensions of spherical polystyrene particles, provided the groundwork for the attribution of the dielectric behavior of cellular suspensions to membrane effects in the $\alpha$ dispersion [53,54]. Work presented in [53] found similar dielectric behavior between the latex spheres and suspensions of fat particles. While spectral data were not presented in [53], the addition of hollow polymer spheres of diameter $0.4 \mu \mathrm{m}$ to an electrolyte medium was found to modify the harmonic pattern [32]. This suggests that the yeast cells contribute to the biologically generated harmonics at a macro level. 


\subsection{Compensation Methods}

The analysis in this thesis work provided strong evidence that interfacial polarization impedance introduces system artifacts, which can mask the biological signal of interest. To compensate for these system artifacts, a method based on the empirical model of [6] was developed. The empirical model was fit to the data to obtain a set of coefficients describing the system behavior over time. The model could then be used to simulate the effect of a linear interfacial polarization impedance, or a constant input amplitude to the system. The case in which the input amplitude was constant was simulated to observe the effect of a constant current in the active case. This constant input amplitude simulation was hampered by the dependance of interfacial impedance on effective current. Results were also obtained when the system response to a linear interfacial polarization impedance was simulated. This method was hampered by the cellular bulk modifying the polarization layer, and as such, some system artifacts remained in the output. Changes to the compensation methods could improve these results, and these possible changes are described in Sec. 7.6. Although these results did not reveal the biological signal of interest, they did provide further evidence that the addition of yeast cells to the electrolyte alters the double layer of charge at the electrode-electrolyte interface.

The reference spectrum method (see Sec. 3.4.1) used in $[3,55,4,5]$, fails to account for the dependance of electrode polarization impedance on effective current. In this thesis, the variation in effective current observed in the active case would cause polarization artifacts to appear in the output of the reference spectrum method. Therefore system artifacts would be correlated with metabolic activity, which would indicate the presence of a biological effect. Work presented in [41] precluded the need for reference spectrum data by using principal components analysis (PCA) to identify systematic variations in the signal. This 
PCA approach treats artifacts as random noise, which may not be the case. Again, since artifacts would be correlated with the effective current variation associated with metabolic activity, erroneous conclusions about a nonlinear biological signal could be made.

The reference spectrum method also fails to account for the change in interfacial impedance due to the addition of cellular bulk to the system. In this thesis, and as was suggested in [7], the addition of yeast to the electrolyte was found to alter the interfacial impedance. A hypothesis of this thesis; that cell movement alters the interfacial polarization layer, means that the harmonic content can vary with metabolically-induced cell movement. As this effect is not accounted for in applying the reference spectrum method, the output would erroneously indicate a characteristic harmonic pattern due to some biological effect.

The two shortcomings of the reference spectrum method described here suggest that harmonic signatures attributed to the transmembrane proteins in the literature, may instead be due to interfacial polarization artifacts.

\subsection{NLDS As a Noninvasive Method}

The electrodes used in the experimental work of this thesis were immersed in a suspension of biological cells. In probing the status of the biological cells it was not necessary for the electrodes to penetrate the cell. Given the lack of penetration of the cell membrane, this application of NLDS can be considered noninvasive.

To transform the application of NLDS from single celled organisms such as yeast to a more complex multi-celled organism such as a human, different approaches are possible. One possible invasive approach would be to extract a blood sample and immerse the electrodes in the blood, as was done in [5]. A noninvasive implementation could be done using surface mounted electrodes such as those commonly employed in electrocardiogram 
work. Considerable future work would likely be necessary to compensate for the added complexity of probing different tissue types in this implementation.

\subsection{Future Work}

A motivating factor in this thesis was to evaluate NLDS as a sensor modality in the in vivo noninvasive determination of blood glucose concentration. The state of the art in this research area (see Chapter 2) has not yet advanced to a point where noninvasive devices are commercially available. A number of sensing modalities have shown promise in a controlled laboratory environment, but taking these methods from the laboratory to a final product will require some significant obstacles to be overcome.

A multi-sensor data fusion approach is one possible means of arriving at a noninvasive solution for determining glucose using existing sensor technology. In this framework, a prediction of blood glucose concentration can be made by combining and processing data from multiple different sensors. No one sensor in particular provides the solution, rather the prediction confidence level is improved by bringing in more sources of information. It was thought that NLDS could provide an additional data stream within the multi-sensor data fusion framework. The electrode system used in NLDS would be compatible with a conventional bioimpedance platform, thereby sharing resources to reduce costs. However, the work of this thesis has indicated that the nonlinear nature of electrode processes contribute significant artifacts which appear in the recorded data. A nonlinear biological response due to transmembrane proteins has proven to be difficult to isolate from these artifacts. The risk in predicting glucose concentration from harmonic content, thought to be associated with cellular metabolic activity, is that the prediction will be based on artifacts.

More work needs to be done to determine how to measure a nonlinear transmembrane 
response to a perturbating electric field, and there are a number of research options.

Given that electrode polarization artifacts confound measuring the biological signal of interest, two classes of methods to rectify this problem are apparent. One class of methods is to employ an electrode-based system and compensate for polarization artifacts. This was the approach used in Chapter 6. These novel compensation methods require improvement, to ensure that the dependance of interfacial polarization on effective current is properly accounted for. Rather than altering the compensation method, one possible means to improve this method is to use a current source, as opposed to a voltage source, at the drive electrodes. This would fix the polarization artifacts at a constant level, preventing them from fluctuating with the observed current variation.

Another aspect of the compensation method which requires improvement is how the method accounts for change in the interfacial polarization impedance caused by the addition of cellular bulk to the electrolyte. The apparatus could be improved by providing a separate compartment in the test container to isolate the cells from the drive electrodes. This compartment could be created using a membrane which allows ions to pass freely, but is impermeable to biological cells. Cellular material could be contained between the recording electrodes, thus not disturbing the polarization layer at the drive electrode-electrolyte interface. Ionic conduction could then proceed unimpeded. This modification could enable the electrode polarization compensation method of Sec. 6.4 to reveal a biological response.

A third approach that could be considered when compensating for polarization nonlinearity is to apply a perturbating voltage input that cancels the polarization nonlinearity. This could be done using an arbitrary voltage waveform generator programmed to generate harmonics which are $180^{\circ}$ out of phase with the harmonics resulting from the interfacial polarization impedance. This concept requires a more in depth understanding of electrode 
polarization effects, but could be applicable to solving the problems caused by electrode polarization artifacts in a variety of applications.

The second class of methods that could be used to prevent polarization artifacts from masking the biological response are those methods which use a sensing strategy other than electrodes. Recently, a high-sensitivity magnetic field detector has been used in place of the recording electrodes in the NLDS apparatus of [21]. This apparatus avoids the possibility of the recording electrodes being externally polarized either through leakage currents, or through immersion in the conductive path. However, this does not improve the more significant problem of polarization artifacts occurring in the stimulus current. Further research which examines other possible means of eliciting and measuring $\mathrm{H}^{+}$ATPase oscillations is required.

\subsection{Summary}

This thesis has contributed to the body of knowledge of NLDS, but must be placed in the context of the motivation; noninvasive blood glucose concentration measurement methods. In this regard, considerable future work is required to determine if a transmembrane signal indicative of metabolic state can be practically measured. If such a signal can be measured, more work will be necessary to transform the knowledge gained from single cell organisms to the human body.

Lessons learned in this work are applicable to one sensor modality which may prove useful in glucose determination; namely bioimpedance. The nonlinearity of polarization impedance can present problems in accurately measuring the impedance of biological tissues. This thesis provides a thorough background in understanding the effects of this nonlinearity, and the method developed to compensate for polarization impedance effects in 
the recorded voltage signals may have some merit in this regard. 


\section{References}

[1] K. Larin, M. Motmedi, T. Ashitkov, and R. Esenaliev. Specificity of noninvasive blood glucose sensing using optical coherence tomography technique: A pilot study. Physics in Medicine \& Biology, 48:1371-1390, 2003.

[2] O. Khalil. Spectroscopic and clinical aspects of noninvasive glucose measurements. Clinical Chemistry, 45(2):165-177, 1999.

[3] A.M. Woodward and D.B. Kell. On the non-linear dielectric properties of biological systems Saccharomyces cerevisiae. Bioelectrochemistry and Bioenergetics, 24:83$100,1990$.

[4] A.M. Woodward and D.B. Kell. On the relationship between the nonlinear dielctric properties and respiratory activity of the obligately aerobic bacterium Micrococcus luteus. Bioelectrochemistry and Bioenergetics, 26:423-439, 1991.

[5] A.M. Woodward, A. Jones, Z. Xin-zhu, J. Rowland, and D.B. Kell. Rapid and noninvasive quantification of metabolic substrates in biological cell suspensions using non-linear dielectric spectroscopy with multivariate calibration and artificial neural networks. principles and applications. Bioelectrochemistry and Bioenergetics, 40:99$132,1996$.

[6] H.P. Schwan. Electrode polarization impedance and measurements in biological materials. Annals of the New York Academy of Science, 148(1):191-209, 1968.

[7] M.J. Hutchings, B.C. Blake-Coleman, and P. Silley. Harmonic "signatures" of microorganisms. Biosensors and Bioelectronics, 9:213-242, 1994.

[8] C.J. McLellan, A.D.C. Chan, and R.A. Goubran. Aspects of nonlinear dielectric spectroscopy of biological cell suspensions. In Proceedings of 28th Annual International IEEE-EMBS Conference, pages 455-458, 2006. 
[9] B. Bode, H. Sabbah, and P. Davidson. What's ahead in glucose monitoring? New techniques hold promise for improved ease and accuracy. Postgraduate Medicine, 109(4):41-49, 2001.

[10] M. Franciosi, F. Pellegrini, G. De Berardis, M. Belfiglio, D. Cavaliere, B. Di Nardo, S. Greenfield, S. Kaplan, M. Sacco, G. Tognoni, M. Valentini, and A. Nicolucci. The impact of blood glucose self-monitoring on metabolic control and quality of life in type 2 diabetic patients: An urgent need for better educational strategies. Diabetes Care, 24(11):1870-1877, 2001.

[11] A. Briggs and S. Cornell. Self-monitoring blood glucose (SMBG): Now and the future. Journal of Pharmacy Practice, 17(1):29-38, 2004.

[12] Z. Zhao. Pulsed photoacoustic techniques and glucose determination in human blood and tissue. $\mathrm{PhD}$ thesis, Dept. of Electrical Engineering and Infotech Oulu, University of Oulu, Oulu, Finland, 2002.

[13] G. Coté. Noninvasive and minimally-invasive optical monitoring technologies. The Journal of Nutrition, 131(5):1596S-1604S, 2001.

[14] S. Malin, T. Ruchti, T. Blank, S. Thennadil, and S. Monfre. Noninvasive prediction of glucose by near-infrared diffuse reflectance spectroscopy. Clinical Chemistry, 45(9):1651-1658, 1999.

[15] H. Heise. Technology for non-invasive monitoring of glucose. Engineering in Medicine and Biology Society, 1996. Bridging Disciplines for Biomedicine. Proceedings of the 18th Annual International Conference of the IEEE, 5:2159-2161, 1996.

[16] K. Maruo, M. Tsurugi, M. Tamura, and Y. Ozaki. In vivo noninvasive measurement of blood glucose by near-infrared diffuse-reflectance spectroscopy. Applied Spectroscopy, 57(10):1236-1244, 2003.

[17] E. Hanlon, R. Manoharan, T. Koo, K. Shafer, J. Motz, M. Fitzmaurice, J. Kramer, I. Itzkan, R. Dasari, and M. Feld. Prospects for in vivo Raman spectroscopy. Physics in Medicine \& Biology, 45:R1-R59, 2000.

[18] D. Stuart, J. Yuen, N. Shah, O. Lyandres, C. Yonzon, M. Glucksberg, J. Walsh, and R. VanDuyne. In vivo glucose measurement by surface-enhanced raman spectroscopy. Analytical Chemistry, 78(20):7211-7215, 2006.

[19] Q. Wan, G. Coté, and J. Dixon. Dual-wavelength polarimetry for monitoring glucose in the presence of varying birefringence. Journal of Biomedical Optics, 10(2):024029, 2005. 
[20] M. Kinnunen and R. Myllylä. Effect of glucose on photoacoustic signals at the wavelengths of 1064 and $532 \mathrm{~nm}$ in pig blood and intralipid. Journal of Physics D: Applied Physics, 38(15):2654-2661, 2005.

[21] J.R. Claycomb, C. Prodan, D. Nawarathna, and J.H. Miller Jr. Nonlinear dielectric spectroscopy of living cell suspensions. In Proceedings of Second Joint EMBS/BMES Conference, pages 1669-1670, 2002.

[22] H.P. Schwan and C.D. Ferris. Four-electrode null techniques for impedance measurement with high resolution. The Review of Scientific Instruments, 39(4):481-485, 1968 .

[23] U. Inan and A. Inan. Electromagnetic Waves. Prentice Hall, 1999.

[24] K.R. Foster and H.P. Schwan. Dielectric properties of tissues and biological materials: a critical review. Critical Reviews in Biomedical Engineering, 17(1):25-104, 1989.

[25] R. Pethig and D.B. Kell. The passive electrical properties of biological systems: their significance in physiology, biophysics and biotechnology. Physics in Medicine and Biology, 32(8):933-970, 1987.

[26] H.P. Schwan. Electrical properties of tissue and cell suspensions. Advances in biological and medical physics, 5:147-209, 1957.

[27] R.D Astumian and B. Robertson. Non-linear effect of an oscillating electric field on membrane proteins. The Journal of Chemical Physics, 91(8):4891-4901, 1989.

[28] W. Kühlbrandt, J. Zeelen, and J. Dietrich. Structure, mechanism, and regulation of the Neurospora plasma membrane $\mathrm{H}^{+}$ATPase. Science, 297:1692-1696, 2002.

[29] J. Blanpain, M. Ronjat, P. Supply, J. Dufour, A. Goffeau, and Y. Dupont. The yeast plasma membrane $\mathrm{H}^{+}$ATPase. The Journal of Biological Chemistry, 267(6):3735$3740,1992$.

[30] T.Y. Tsong and R.D Astumian. Absorption and conversion of electric field energy by membrane bound ATPases. Bioelectrochemistry and Bioenergetics, 15:457-476, 1986.

[31] D.B. Kell and A.M. Woodward. On harmonic generation in non-linear biological systems. Biosensors and Bioelectronics, 10:639-641, 1995.

[32] B.C. Blake-Coleman and M.J. Hutchings. Harmonic generation in "non-linear" biological systems. Biosensors and Bioelectronics, 9:99-103, 1994. 
[33] J.-P. Diard, B. Le Gorrec, and C. Montella. Impedance measurement errors due to non-linearities-I. low frequency impedance measurements. Electrochimica Acta, 39(4):539-536, 1996.

[34] M.R. Neuman. Medical Instrumentation Application and Design, chapter Biopotential Electrodes. John Wiley and Sons, New York City, third edition, 1998.

[35] S. Grimnes and $\emptyset$. Martinsen. Bioimpedance and Bioelectricity Basics. Academic Press, London, 2000.

[36] M. Moussavi, H.P. Schwan, and H.H. Sun. Harmonic distortion caused by electrode polarization. Biological Engineering and Computing, 32:121-125, 1994.

[37] P. Mirtaheri, S. Grimnes, and $\varnothing$. Martinsen. Electrode polarization impedance in weak $\mathrm{NaCl}$ aqueous solutions. IEEE Transactions on Biomedical Engineering, 52(12):2093-2099, 2005.

[38] H.P. Schwan, E.T. McAdams, and J. Jossinet. Sauer's non-linear voltage division. Medical \& Biological Engineering and Computing, 40:542-545, 2002.

[39] A.M. Woodward, E.A. Davies, S. Denyer, C. Oliff, and D.B. Kell. Non-linear dielectric spectroscopy: antifouling and stabilization of electrodes by a polymer coating. Bioelectrochemistry and Bioenergetics, 51:13--20, 2000.

[40] J.G. Proakis and D.G. Manolakis. Digital Signal Processing. Prentice Hall, third edition, 1996.

[41] A. Jones. Computational aspects of nonlinear biological dielectric spectroscopy. $\mathrm{PhD}$ thesis, Dept. of Computer Science, University of Wales, Aberystwyth, Wales, 2001.

[42] H.P. Schwan. Linear and non-linear electrode polarization and biological materials. Annals of Biomedical Engineering, 20:269-288, 1992.

[43] D.B. Kell, A.M. Woodward, E.A. Davies, R.W. Todd, M.F. Evans, and J.J. Rowland. Non-linear dielectric spectroscopy of biological systems: Principles and applications. Kluwer Academic Publishers.

[44] N. Saris, H. Holkeri, R. Craven, C. Stirling, and M. Makarow. The Hsp70 homologue Lhs $1 p$ is involved in a novel function of the yeast endoplasmic reticulum, refolding and stabilization of heat-denatured protein aggregates.

[45] H. Schmidt and R. Machan. Continuous Eh-recordings in sediment columns. Helgoland Marine Research, 27(1):101-107, 1975. 
[46] K. Darowicki. Linearization in impedance measurements. Electrochimica Acta, 42(12):1781-1788, 1997.

[47] L.W. Couch II. Digital and Analog Communication Systems. Prentice Hall, sixth edition, 2001.

[48] J. McMurry and R.C. Fay. Chemistry. Prentice Hall, 1995.

[49] P. Mirtaheri, S. Grimnes, $\varnothing$. Martinsen, and T.I. Tønnessen. A new biomedical sensor for measuring $\mathrm{PCO}_{2}$. Physiological Measurement, 25:421-436, 2004.

[50] K. Asami, E. Gheorghiu, and T. Yonezawa. Real-time monitoring of yeast cell division by dielectric spectroscopy. Biophysical Journal, 76:3345-3348, 1999.

[51] H.P. Schwan and B. Onaral. Linear and nonlinear properties of platinum electrode polarisation III: Equivalence of frequency-and time-domain behaviour. Medical \& Biological Engineering and Computing, 23(1):28-32, 1985.

[52] A. Richardot and E.T. McAdams. Harmonic analysis of low-frequency bioelectrode behavior. IEEE Transactions on Medical Imaging, 21(6):604-612, 2002.

[53] H.P. Schwan, G. Schwarz, J. Maczuk, and H. Pauly. On the low-frequency dielectric dispersion of colloidal particles in electrolyte solution. Journal of Physical Chemistry, 66:2626-2635, 1962.

[54] G. Schwarz. A theory of the low-frequency dielectric dispersion of colloidal particles in electrolyte solution. Journal of Physical Chemistry, 66:2636-2642, 1962.

[55] A.M. Woodward and D.B. Kell. Dual frequency excitation: a novel method for probing the nonlinear dielectric properties of biological systems, and its application to suspensions of $S$. cerevisiae. Bioelectrochemistry and Bioenergetics, 25:395-413, 1990. 